\title{
Associated Particle Tagging (APT) in Magnetic Spectrometers
}

David V. Jordan, James E. Baciak, Sean C. Stave

Pacific Northwest National Laboratory

David Chichester

Idaho National Laboratory

Daniel Dale, Yujong Kim, Frank Harmon

Idaho State University/Idaho Accelerator Center

October 2012 


\title{
DISCLAIMER
}

This report was prepared as an account of work sponsored by an agency of the United States Government. Neither the United States Government nor any agency thereof, nor Battelle Memorial Institute, nor any of their employees, makes any warranty, express or implied, or assumes any legal liability or responsibility for the accuracy, completeness, or usefulness of any information, apparatus, product, or process disclosed, or represents that its use would not infringe privately owned rights. Reference herein to any specific commercial product, process, or service by trade name, trademark, manufacturer, or otherwise does not necessarily constitute or imply its endorsement, recommendation, or favoring by the United States Government or any agency thereof, or Battelle Memorial Institute. The views and opinions of authors expressed herein do not necessarily state or reflect those of the United States Government or any agency thereof.

\author{
PACIFIC NORTHWEST NATIONAL LABORATORY \\ operated by \\ BATTELLE \\ for the \\ UNITED STATES DEPARTMENT OF ENERGY \\ under Contract DE-AC05-76RL01830
}

Printed in the United States of America
Available to DOE and DOE contractors from the Office of Scientific and Technical Information,
P.O. Box 62, Oak Ridge, TN 37831-0062;
ph: (865) 576-8401
fax: $(865)$ 576-5728
email: reports@adonis.osti.gov

\begin{abstract}
Available to the public from the National Technical Information Service, U.S. Department of Commerce, 5285 Port Royal Rd., Springfield, VA 22161 ph: (800) 553-6847 fax: $(703) 605-6900$ email: orders@ntis.fedworld.gov online ordering: http://www.ntis.gov/ordering.htm
\end{abstract}

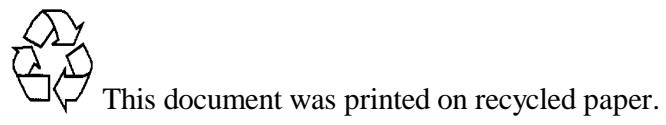




\section{Associated Particle Tagging (APT) in Magnetic Spectrometers}

David V. Jordan, James E. Baciak, Sean C. Stave

Pacific Northwest National Laboratory

David Chichester

Idaho National Laboratory

Daniel Dale, Yujong Kim, Frank Harmon

Idaho State University/Idaho Accelerator Center

October 2012

Prepared for the Department of Energy

Office of Non-Proliferation Research and Development (NA-22) under U.S. Department of Energy Contract DE-AC05-76RL01830

Pacific Northwest National Laboratory

Richland, Washington 99352

*Corresponding author: David.Jordan@pnnl.gov 


\section{Summary In Brief}

The Associated Particle Tagging (APT) project, a collaboration of Pacific Northwest National Laboratory (PNNL), Idaho National Laboratory (INL) and the Idaho State University (ISU)/Idaho Accelerator Center (IAC), has completed an exploratory study to assess the role of magnetic spectrometers as the linchpin technology in next-generation tagged-neutron and tagged-photon active interrogation (AI). The computational study considered two principle concepts: (1) the application of a solenoidal alpha-particle spectrometer to a next-generation, large-emittance neutron generator for use in the associated particle imaging technique, and (2) the application of tagged photon beams to the detection of fissile material via active interrogation. In both cases, a magnetic spectrometer momentum-analyzes charged particles (in the neutron case, alpha particles accompanying neutron generation in the D-T reaction; in the tagged photon case, post-bremsstrahlung electrons) to define kinematic properties of the relevant neutral interrogation probe particle (i.e. neutron or photon). The main conclusions of the study can be briefly summarized as follows:

Neutron generator:

- For the solenoidal spectrometer concept, magnetic field strengths of order 1 Tesla or greater are required to keep the transverse size of the spectrometer smaller than 1 meter. The notional magnetic spectrometer design evaluated in this feasibility study uses a 5-T magnetic field and a borehole radius of $18 \mathrm{~cm}$.

- The design shows a potential for 4.5 Sr tagged neutron solid angle, a factor of 4.5 larger than achievable with current API neutron-generator designs.

- The potential angular resolution for such a tagged neutron beam can be less than $0.5^{\circ}$ for modest Si-detector position resolution (3 mm). Further improvement in angular resolution can be made by using Si-detectors with better position resolution.

- The report documents several features of a notional generator design incorporating the alpha-particle spectrometer concept, and outlines challenges involved in the magnetic field design.

Tagged photon interrogation:

- We investigated a method for discriminating fissile from benign cargo-material response to an energy-tagged photon beam. The method relies upon coincident detection of the tagged photon and a photoneutron or photofission neutron produced in the target material. The method exploits differences in the shape of the neutron production cross section as a function of incident photon energy in order to discriminate photofission yield from photoneutrons emitted by non-fissile materials. Computational tests of the interrogation method as applied to material composition assay of a simple, multi-layer target suggest that the tagged-photon information facilitates precise (order 1\% thickness uncertainty) reconstruction of the constituent thicknesses of fissile (uranium) and high-Z $\mathrm{Pb}$ ) constituents of the test targets in a few minutes of photon-beam exposure. We assumed an 18-MeV endpoint tagged photon beam for these simulations.

- The report addresses several candidate design and data analysis issues for beamline infrastructure required to produce a tagged photon beam in a notional AI-dedicated facility, including the accelerator and tagging spectrometer. 


\section{Executive Summary}

Pacific Northwest National Laboratory (PNNL), in collaboration with Idaho National Laboratory (INL, Dr. David Chichester) and the Idaho State University (ISU)/Idaho Accelerator Center (IAC, Dr. Daniel Dale), has completed an exploratory (one-year) feasibility study aimed at assessing the role of magnetic spectrometers as the linchpin technology in next-generation tagged-neutron and tagged-photon active interrogation (AI). The study addressed two principle concepts via computational modeling and notional design assessments:

1. Application of the solenoidal spectrometer to the development of a next-generation, large-emittance electronic neutron generator (ENG) for the associated particle imaging (API) active interrogation technique;

2. Application of photon tagging to photonuclear detection of special nuclear materials (SNM).

PNNL led a modeling-based computational investigation of both concepts. External project collaborators at INL and ISU/IAC supplied technical guidance and conceptual design input on the neutron generator and tagged-photon accelerator beamline infrastructure aspects, respectively. The feasibility assessment quantified, estimated, and/or bounded key performance metrics for AI applications achievable using notional spectrometers for charged-particle detection and momentum analysis. The assessment included scenario-specific performance modeling in an inspection/threat-reduction deployment context. The neutron-generator and tagged-photon studies complemented one another, in the sense that both involve the use of magnetic ion-optical spectrometry as a key ingredient in the proposed interrogation system, but the detailed exploratory objectives differed somewhat in the two cases. In the neutron generator case, the API method is already the subject of ongoing investigations in the AI community, and the proposed spectrometer-based technique aims at substantially improving the performance of the ENG deployed in this state-of-the-art neutron interrogation technique. In the tagged photon case, the situation is qualitatively different. Although the energy-tagged photon method is a staple of the nuclear physics community, where tagging facilitates energy-dependent measurements of e.g. reaction cross sections, the potential utility of the energy specificity afforded by a tagged photon beam method has not been previously addressed in the AI community. Thus this study constitutes an initial foray into quantifying the "value added" of the tagged-photon approach for threat-reduction applications such as non-destructive assay (NDA) of containerized materials.

The neutron-generator research evaluated a conceptual design for a next-generation electronic neutron generator (ENG) incorporating a novel, magnetic-spectrometer based detection scheme for alpha particles produced in the deuterium-tritium (D-T) reaction that provides the source of fast (14 MeV) neutron generation. The primary research objective was to substantially improve neutron tagging efficiency, i.e. the fraction of neutrons generated in the D-T reaction that can be usefully exploited as interrogation probes, for ENGs used for API. We addressed this objective by designing and simulating the performance of a notional, large-angle spectrometer for detection and momentum analysis of the alpha particle emitted in the $\mathrm{D}+\mathrm{T} \rightarrow$ alpha $+\mathrm{n}$ reaction. We incorporated the model of the notional ENG in a simulation of cargo interrogation via inelastic scattering of tagged fast neutron, followed by coincident detection of nuclear deexcitation gamma-rays. Collaboration with INL suggested some specific features of a candidate 
ENG design incorporating the spectrometric tagging method, and highlighted some potential implementation challenges and opportunities.

The photon-tagging research scope included an information-content analysis of the "valueadded” for special nuclear material (SNM) detection techniques exploiting a tagged energy resolution two to three orders of magnitude finer than that possible with conventional, broadspectrum (i.e., untagged) bremsstrahlung photon beams. The application of energy-tagged bremsstrahlung photons to AI offers a potentially powerful means of discriminating fissile from non-fissile materials by mapping the energy dependence of the photoneutron yield over the energy range 6 to $20 \mathrm{MeV}$, separating the relative contributions of the photofission and photoneutron (giant dipole) processes. Measurements at the peak of the photofission cross section would reduce the photon dose required to discriminate high- $\mathrm{Z}$ from fissile materials. The research methodology included simulation of photoneutron emission using tagged photons. The main performance-assessment objective in these simulation studies was an estimate of the threatmaterial discrimination capability afforded by exploiting the probe-beam energy specificity in the tagging method. Collaboration with ISU/IAC addressed potential electron beamline infrastructure requirements for implementing a photon-tagging facility, with particular reference to physics measurements and measurement campaigns aimed at further quantifying the utility of the method for threat-reduction applications.

Both aspects of the study included a preliminary assessment of some, although not all, practical implementation issues, and both identified options for conceptual design of key components and/or infrastructure required for an AI system. At the conclusion of this exploratory feasibility study, we have attempted to provide sufficient indication of the utility of the role of the magnetic spectrometer as the linchpin sub-system in energy- and/or direction-tagged neutral particle sources to permit rational, defensible assessment of the prospects for further research and development in the implementation of these approaches. We briefly summarize key conclusions of the two aspects of the research below.

Neutron API: In the associated particle imaging (API) technique for neutron generation, detection of the outgoing triton ( $\mathrm{D}+\mathrm{D}$ reaction) or alpha particle (D+T reaction) and measurement of its direction, in conjunction with 2-body reaction kinematics, tags the outgoing neutron (emitted back-to-back relative to the charged product in the center-of-mass system) and determines its direction to within a finite, but typically small $\left(\sim 1^{\circ}\right)$, angular resolution. Conventional applications of the technique use a position-sensitive detector (e.g. a fiber-optic face plate coated with a thin layer of inorganic scintillator) to register the charged reaction product [Chichester 2005]. The fraction of the (approximately isotropic) neutron angular distribution tagged thus depends on the area of the charged-particle detector and on its standoff distance from the location of the fusion reaction in the target. Currently achievable tagged neutron solid angles are approximately 1 steradian (Sr).

The present project explores application of the solenoidal spectrometer technique [Wuosmaa 2007] to API in an effort to substantially increase the solid angle coverage of the charged reaction product (and thus, of the tagged neutron beam). The operating principle is indicated in Figure S1 below. A homogenous magnetic field of magnitude $B$ fills the interior of the spectrometer and is aligned parallel to its longitudinal axis. The primary particle beam (e.g. deuterium ions) enters the spectrometer along the axis and impinges upon the target. In general 
this beam will have a small spot size ( few mm), and typical fusion reactions will occur very close to the axis. Charged-particle emission from reactions in the target will have transverse $(V \perp)$ and longitudinal $\left(V_{\|}\right)$velocity components. The combination of circular motion in the transverse plane (with radius $r=m V \perp / q e B$, where $m$ and $q$ are the particle mass and charge, respectively) and rectilinear motion with constant velocity parallel to the axis produces a helical trajectory that returns the charged particle to the axis after one revolution in the transverse plane. Because this motion occurs at a fixed frequency of revolution (namely, the cyclotron frequency of the particle in the magnetic field), the time required to return to the spectrometer axis is a constant, independent of the particle's momentum vector upon exiting from the target.

A hollow, annular, position-sensitive charged-particle detector (e.g. a pixilated silicon detector array) is located on the spectrometer axis either upstream or downstream of the target (or both). The detector records the longitudinal position at which the charged particle returns to the spectrometer axis. Combined with the (known) transit time, this position measurement yields the component of the particle momentum parallel to the spectrometer axis. Measurement of the particle's kinetic energy (via measurement of the deposited energy in the focal plane detector) then yields the magnitude of the perpendicular component of the particle's momentum. The azimuthal position of the detected event similarly determines the azimuthal emission angle, so that the complete direction vector of the charged reaction product at the target can be reconstructed (and this, in turn, determines the neutron direction vector). The key point is that the spectrometer maps essentially the entire (polar, azimuthal) phase space of the charged reaction product onto the position-sensitive detector, yielding close to $2 \pi$ solid angle tagging coverage for neutron emissions into either the upstream or downstream halves of the angular emission space. A larger neutron tagging fraction means in turn that the generator can be positioned closer to the inspected object while maintaining similar spatial extent of the taggedneutron illumination, reducing the time required for a measurement and increasing effective generator life.

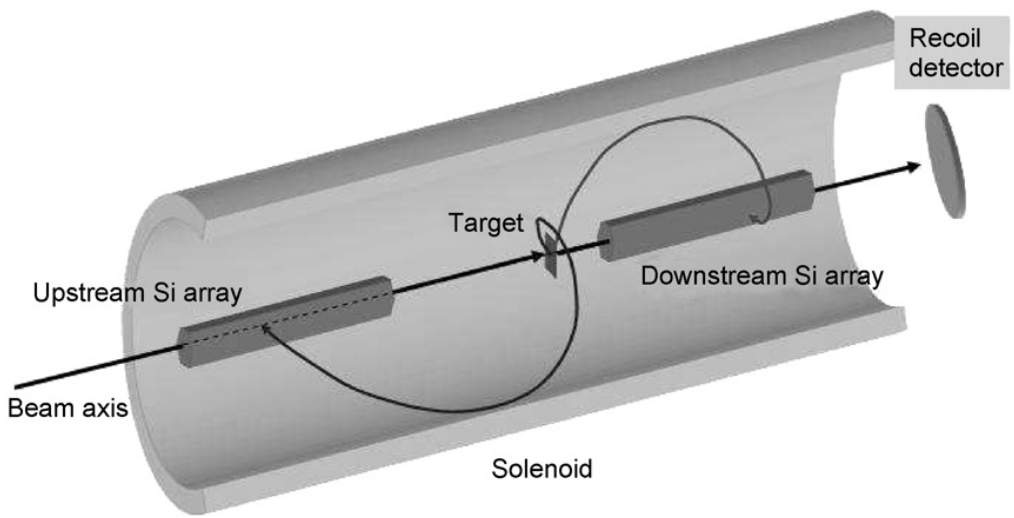

Figure S1. Schematic solenoidal charged-particle spectrometer [Wuosmaa 2007]. In an application of this alpha-spectrometry method to a tagged-neutron generator, only the upstream Si array would be present.

In the feasibility study, PNNL developed a Geant4 radiation transport model of a notional solenoidal spectrometer system for neutron tagging in the D-T reaction. We used this model to investigate the tagged-neutron energy and angular resolution, and the neutron tagging efficiency, as functions of key properties of the spectrometer, including position resolution of the axial 
detector, uniformity of the magnetic field, and diameter of the solenoid. PNNL collaborated with Dr. David Chichester of INL throughout the feasibility study to investigate the technical merits of a variety of notional designs for incorporating a solenoidal- or permanent-magnet, large-angle spectrometer in a D-T generator.

The feasibility study incorporated the magnetic solenoidal alpha-spectrometer concept in a computational-based performance evaluation of a notional tagged-neutron generator for API applications. In the spectrometer model (see Figure S2), an array of Si detectors is placed on the axis of the solenoid. A key advantage of the magnetic spectrometer design for neutron tagging is the capability to tag a larger emission cone of neutrons than is possible with current API neutron generator form factors. The approach currently implemented in API generators incorporates an externally-mounted alpha detector viewing the D-T target through a thin window at a standoff distance on the order of $10 \mathrm{~cm}$. The maximum solid angle of tagged neutrons achievable in this configuration is approximately $1 \mathrm{Sr}$. The external-detector form factor is susceptible to potential breakage of the thin window. We have modeled the D-T reaction in a 5-Tesla magnetic spectrometer to simulate the capability of such a device to tag neutrons. In order to prevent alpha particles from interacting in the walls of the solenoid, the borehole must have at least an $18 \mathrm{~cm}$ radius (but can be reduced with a stronger magnetic field). It is possible in principle to use permanent magnets, rather than electromagnets magnets, but the weaker magnetic field would require a larger system to accommodate the same alpha-particle angular acceptance. The magnetic spectrometer design evaluated in this feasibility study shows a potential for $4.5 \mathrm{Sr}$ tagged neutron solid angle, and further increases may be possible with optimization of the focal plane detector array design.

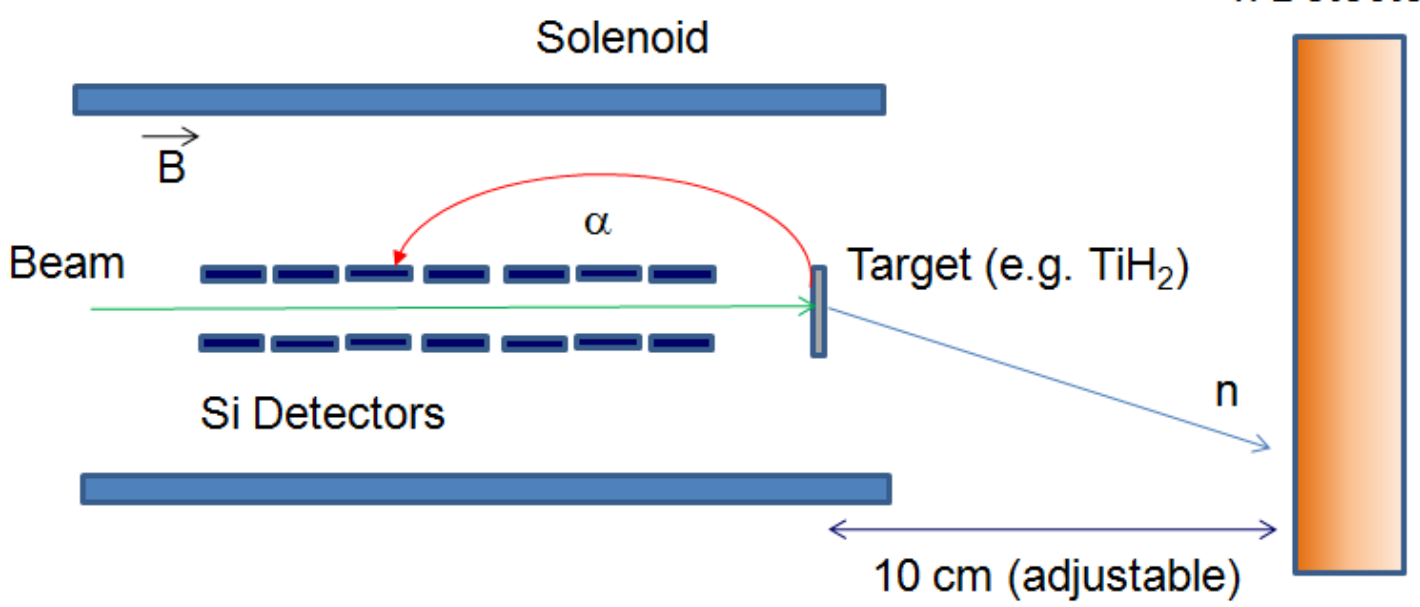

Figure S2. Schematic drawing of simulated tagged-neutron generator incorporating a magnetic spectrometer for alpha-particle detection and momentum analysis (not to scale). A beam of deuterons, incident from the left, bombards a thin metal hydride target and generates neutrons via the $D+T \rightarrow \alpha+n$ reaction. The alpha particle executes helical motion in the axial magnetic field and impinges on solid-state detectors in the spectrometer focal plane. The drawing indicates a cross-section through a cylindrical (or potentially foursided tube) detector array. The distance from the target to the detection point yields information on the emission angle of the alpha at the reaction vertex, and thus the neutron emission angle. 
Note that by incorporating pixelated silicon ( $\mathrm{Si}$ ) solid-state detectors placed on the spectrometer axis, the magnetic spectrometer design for a neutron tagging system no longer requires a fragile, thin-window alpha particle detector. The cost of larger neutron tagging efficiency and enhanced robustness, however, is that the magnetic field requirements make portability a substantial challenge. Magnetic fields on the order of several Tesla are required in order to keep the transverse dimensions of the spectrometer below 1 meter. A superconducting 5-Tesla magnet, to take a specific example, is a substantial piece of fixed-installation infrastructure with characteristic dimensions on the order of 1 meter. Although alternative form factors that use permanent magnets and smaller field strengths may provide useful performance capability, the spectroscopic technique may prove most appropriate for implementation of a facility-based, rather than mobile, next-generation API capability.

An attractive feature of the solenoidal spectrometer's principle of operation is that the cycle time (i.e., the time interval required for a charged particle to cross the axis of the solenoid after emerging from the target) is essentially independent of the particle's energy and direction. This in turn implies that target energy straggling of alphas produced by the D-T reaction will not compromise reconstruction of the tagged neutron direction (and, via 2-body kinematics, the neutron energy), although multiple scattering of the alpha particle as it exits the target will degrade the resolution of the reconstructed neutron kinematics. Using knowledge of current neutron generator/tagging system designs, we incorporated similar design parameters (target geometries, pressures, etc.) to describe alpha-particle scattering effects in our modeled magnetic spectrometer system. Using the energy deposited in the silicon detector array by the alpha particle, along with interaction position, and cycle time (based on the magnetic field), we reconstruct the tagged neutron energy and trajectory. The potential angular resolution for such a tagged neutron can be less than 0.5 degree for modest Si-detector position resolution (3 mm). Further improvement in angular resolution can be made by using Si-detectors with better position resolution.

The project team conducted exploratory scenario modeling to examine the application of neutron tagging to cargo container screening. This initial modeling (see Figure S3) showed that an interrogation system incorporating the magnetic spectrometer-based neutron tagger has the potential to image the position of objects within a cargo container to a position resolution of order $10 \mathrm{~cm}$. The method investigated, inelastic neutron scattering with coincident detection of nuclear de-excitation gamma-rays, also offers the potential to distinguish different types of materials within a container (using standard $\mathrm{NaI}$ detectors to detect the de-excitation gamma rays). However, the neutron-source modeling to date has not quantified this potential performance capability.

In addition to providing additional detail on the computational modeling aspects of the neutron generator research scope, the body of the report outlines a notional design for an ENG incorporating an alpha-particle spectrometer. This design illustrates a strategy for mounting the Si detector array within the generator interior. Several practical considerations involved in implementing the notional design scheme are discussed. 


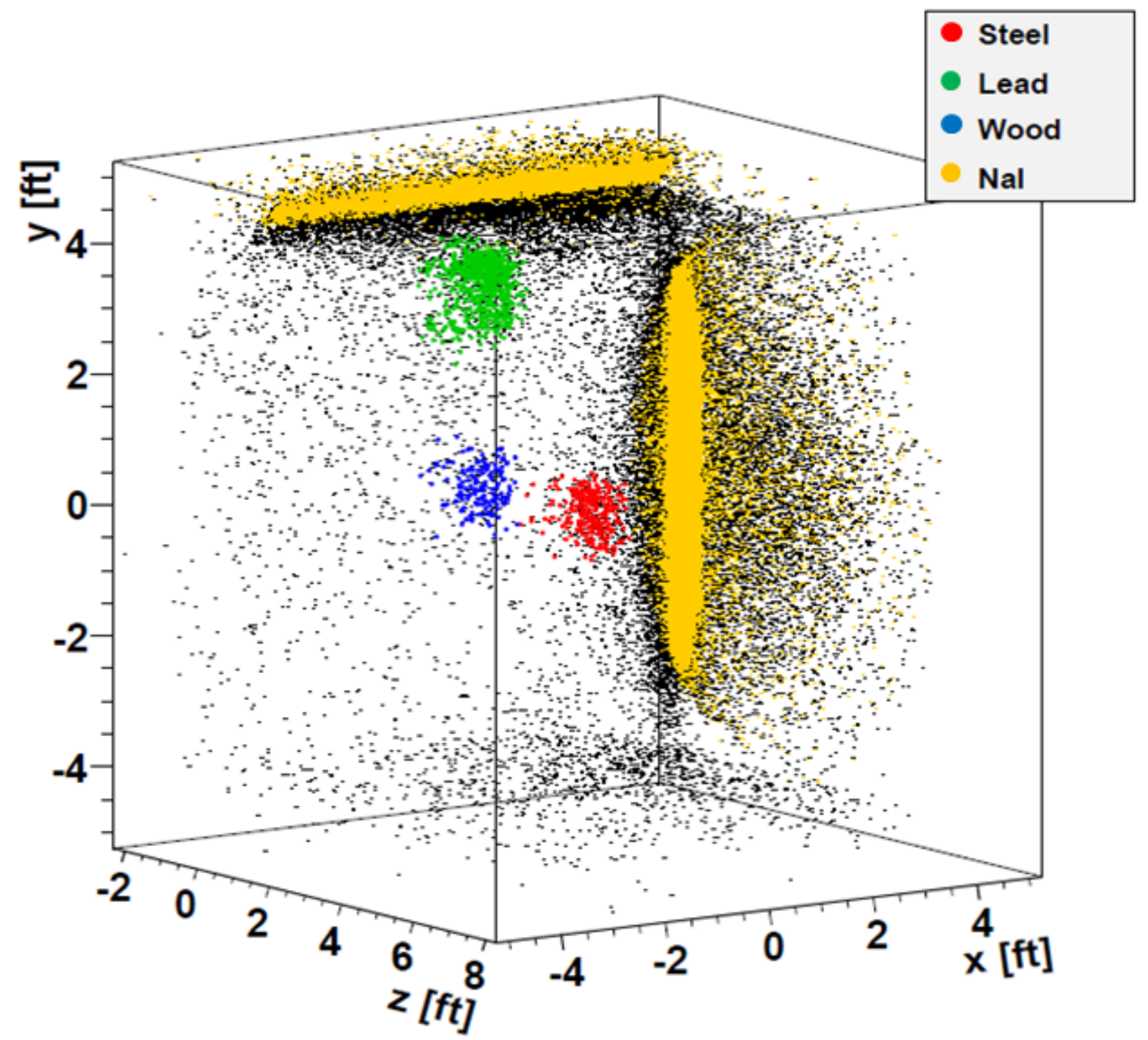

Figure S3. Simulated example application of tagged neutrons for cargo interrogation. The positions of the three objects in the cargo container interior have been reconstructed on the basis of the tagged-neutron kinematics and the detected gamma-ray position and coincidence timing. The blurred yellow bars in the figure suggest the size and position of the arrays of $\mathrm{NaI}$ detectors assumed in the simulation. Black dots correspond to scattering events from materials (e.g. container walls) other than the objects identified in the legend. The steel, lead, and wood items in the container are color enhanced for ease of visualization, but material identification based upon the detected gamma-ray spectrum has not been performed in this simulation.

Photon tagging: Figure S4 below, reproduced from [Vogt 1993], presents a schematic overview of the tagged-photon technique as typically applied to nuclear physics experimentation. An electron beam of energy $E_{0}$ impinges on a thin foil radiator and produces bremsstrahlung photons with a continuum of energies up to $E_{0}$. The electron exits the foil (assumed thin enough to negligibly degrade the electron energy) and is momentumanalyzed in a magnetic spectrometer. Measurement of the electron's energy, $\mathrm{E}_{\mathrm{e}}$, following bremsstrahlung yields the energy of the emitted photon, $E_{\mathrm{g}}$, via $E_{\mathrm{g}}=\mathrm{E}_{\mathrm{o}}-\mathrm{E}_{\mathrm{e}}$. Measurement of the time difference between arrival of the post-bremsstrahlung electron in the spectrometer 
focal plane and detection of the photon permits identification of coincident (electron, photon) pairs. As indicated in the figure, the time-to-digital converter (TDC) spectrum of the (photon-start, electron-stop) time difference typically features a peak corresponding to true coincidences of physically correlated (electron, photon) pairs and a smoothly-varying background of random coincidences. Separate TDC spectra are available for each channel of the tagger. Gating on the "trues" timing window, i.e. the TDC peak region of interest, tags the photon with the energy of the corresponding channel in the electron focal plane. The relative rates of true and random coincidences within this gate determine the probability that the photon energy is measured correctly (i.e., to within the energy resolution of a single channel in the electron spectrometer that is correctly correlated with the photon).

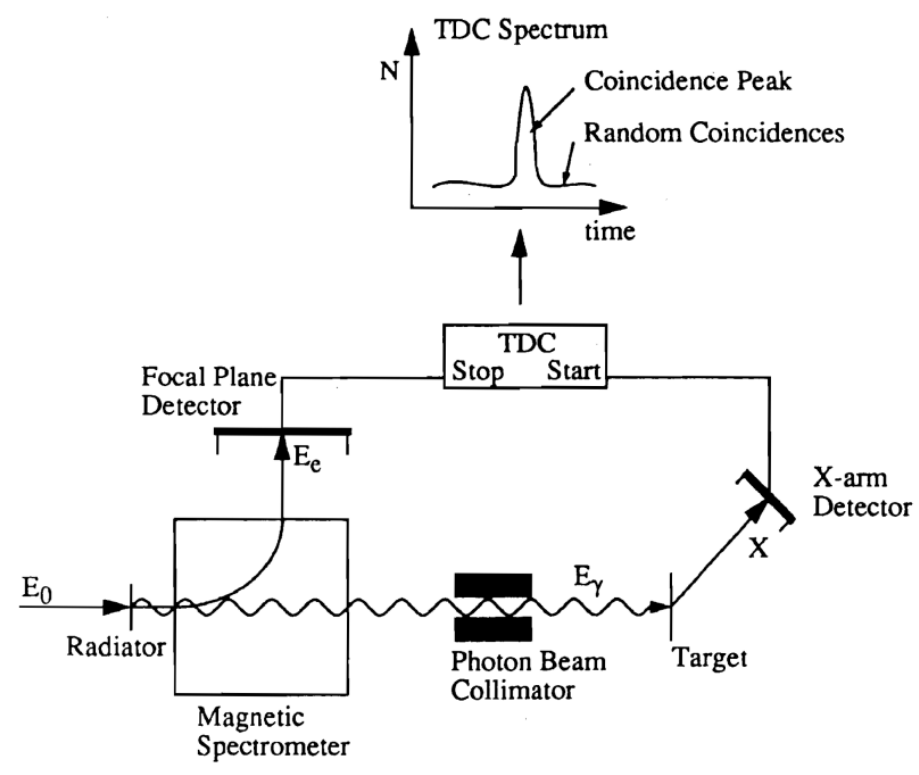

Figure S4. The principle of photon tagging [Vogt 1993].

The photon-tagging scope of the feasibility study addresses the application of the tagging technique as an enabling technology for photonuclear active interrogation applications. The tagged bremsstrahlung technique offers a 1 to 2 order-of-magnitude improvement in the effective photon energy resolution in an active interrogation system, relative to a conventional bremsstrahlung beam. The key properties of a tagged photon beam can be summarized briefly as follows:

- Quasi-monochromatic: A tagged-photon system enables energy specificity on the order of $\pm 90 \mathrm{keV}$ in a hypothetical 50-channel tagger spanning the bremsstrahlung photon energy distribution from 1 to $10 \mathrm{MeV}$.

- Tunable: Both the magnetic field of the electron spectrometer and the position of the electron detector array in the spectrometer focal plane can be varied to fine-tune the set of central photon energies $\left\{E_{i}\right\}$ defined by the tagger channels, with the separation between central energies in adjacent channels determined by the pitch (i.e., spacing) of the electron detector array. 
- Real-time energy-response mapping: Multiple quasi-monoenergetic photon beams are sampled in real time, simultaneously, from the same bremsstrahlung spectrum, without varying the incident electron beam energy.

- Highly reproducible: Because no energy switching of the primary electron beam is required, the reproducibility (in energy and flux) of the quasi-monoenergetic photon beam set $\left\{E_{i}\right\}$ is limited only by the constant-energy stability of the electron linac and the reproducibility of the electron spectrometer's magnetic fields.

The finite response time of spectrometer detectors and pulse-processing electronics limit the effective maximum tagged photon rate relative to a broad-spectrum, untagged bremsstrahlung beam. Thus flux limitations in the photon tagging approach represent a potentially serious technical tradeoff, in comparison to conventional bremsstrahlung and radioisotope sources. An important objective of the exploratory research is to determine the extent to which the additional information content available from the energy specificity of the tagging approach overcomes this flux limitation, for the purposes of SNM detection and identification. Because the tagging method requires coincident detection with postbremsstrahlung electrons in the spectrometer focal plane, the rate of accidental coincidences between "X-arm" detector events (registering e.g. photonuclear products emitted by the inspected item) and electron focal-plane events is a critical limiting factor for photonuclear applications. Vogt et al. [1993] quote an upper limit on the order of $10^{8}$ tagged photons per second in the Saskatchewan Accelerator Laboratory (SAL) tagging facility, a photon flux modest compared to typical COTS X-ray machines $\left(O\left(10^{11}\right)\right.$ photons $/\left(\mathrm{cm}^{2}\right.$-second) at 3 meter standoff).

However, an important advantage of the tagging technique in photonuclear applications is that the energy specificity facilitates precise characterization, relative to broad-spectrum bremsstrahlung, of isotope-specific reaction thresholds. Figures S5 and S6 below illustrate the substantial qualitative difference in cross section shapes for neutron photoproduction on U-235 and Fe-56, according to ENDF/B-VII. (For this example, the ENDF evaluated nuclear data library may include both empirical data and supplementary nuclear models.) Note that the neutron photoemission spectrum from fissile materials will in general include both a Watt-spectrum component (representing the photofission neutron emission spectrum) and a (gamma, n) knockout component. Non-fissile materials will lack the Watt-spectrum contribution to the yield distribution. The dramatic difference in response shapes suggests that discrimination of these materials may be accomplished by examining the dependence of the fast neutron yield on both photon energy and neutron energy. Note that these energydependent emission yields will be much more difficult to measure reliably with a conventional bremsstrahlung beam, in which the detection system unavoidably integrates the nuclear response over the broad photon energy distribution. 


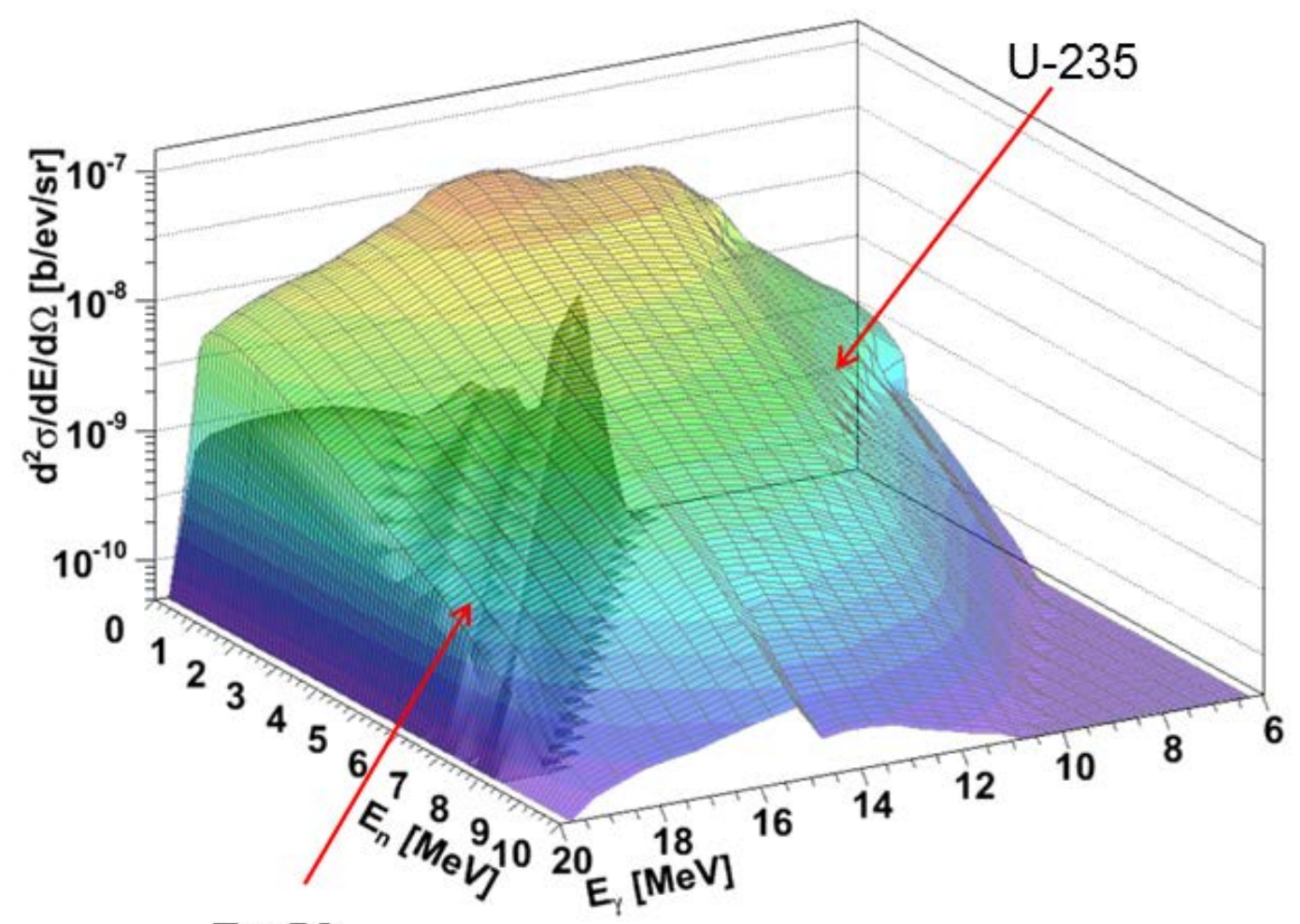

Fe-56

Figure S5. Comparison of ENDF/B-VII differential (gamma, n) cross sections for ${ }^{235} \mathrm{U}$ and ${ }^{56} \mathrm{Fe}$ as a function of photon and neutron energy.

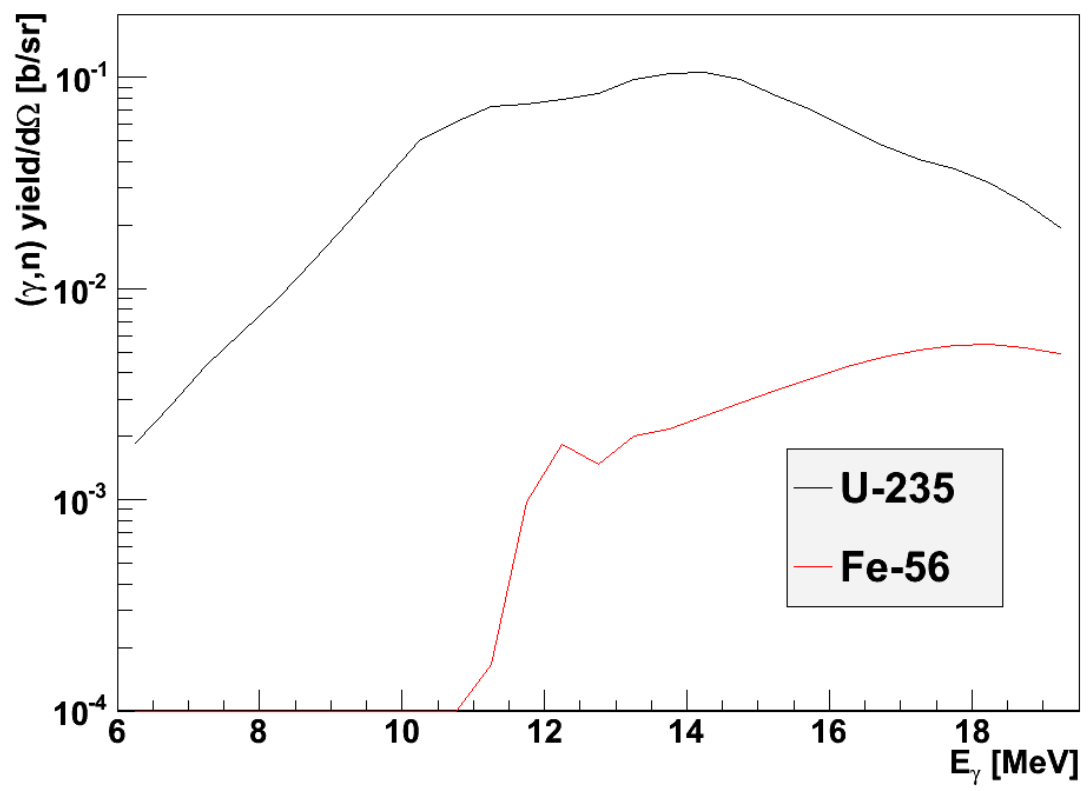

Figure S6. Projection of the ENDF/B-VII differential (gamma, n) yields in Figure S5 on the photon-energy axis. Note that for $10 \mathrm{MeV}$ photons, the neutron yield for ${ }^{235} \mathrm{U}$ is over 1000 times larger than for ${ }^{56} \mathrm{Fe}$. 
In the feasibility study, PNNL performed computational assessments of the photon-tagging approach to evaluate the "value added" of the photon energy specificity. We examined variation of detected neutron counts in a notional tagged photon system (see Figure S7) incorporating fastneutron detection and time-of-flight neutron spectroscopy. The simulations take into account the shape of the photo-produced neutron response as a function of initial photon energy and neutron energy, and the ability to map these responses with measurements at finite resolution in the tagged photon energy and neutron time-of-flight. PNNL's simulation results (see Figure S8) indicate that, given a limited database of known "library" isotopes, the neutron distributions in the ( $\left.E_{\text {gamma }}, E_{\text {neutron }}\right)$ response plane are sufficiently different for fissile and non-fissile materials that it is feasible to determine the type and relative amounts of material in a simple "sandwich target” configuration for the inspected item. This is accomplished by fitting linear combinations of the known response shapes to the measured response. The simulations take into account, and the materials-discrimination analysis algorithm assumes knowledge of, realistic detector efficiencies, solid angles and thresholds. PNNL has evaluated both "one-dimensional” (i.e. gamma-ray energy cross section shape integrated over the detected fast neutron spectrum) and "two-dimensional" (i.e., exploiting the cross section shape differences in both gamma-ray and neutron energies) approaches to system response characterization. We have used both approaches to successfully fit and reconstruct the amount and type of material present in a particular inspection scenario, as a function of dwell time. Detailed comparisons of the two approaches, and a "value-added" analysis of the availability of the full response information in the ( $\left.E_{\text {gamma }}, E_{\text {neutron }}\right)$ plane relative to the "one-dimensional” integrated neutron response vs. $E_{\text {gamma, }}$ are discussed in the body of the report. The simulation and analysis approach adopted for the feasibility study readily accommodates additional isotopes and other scenarios.

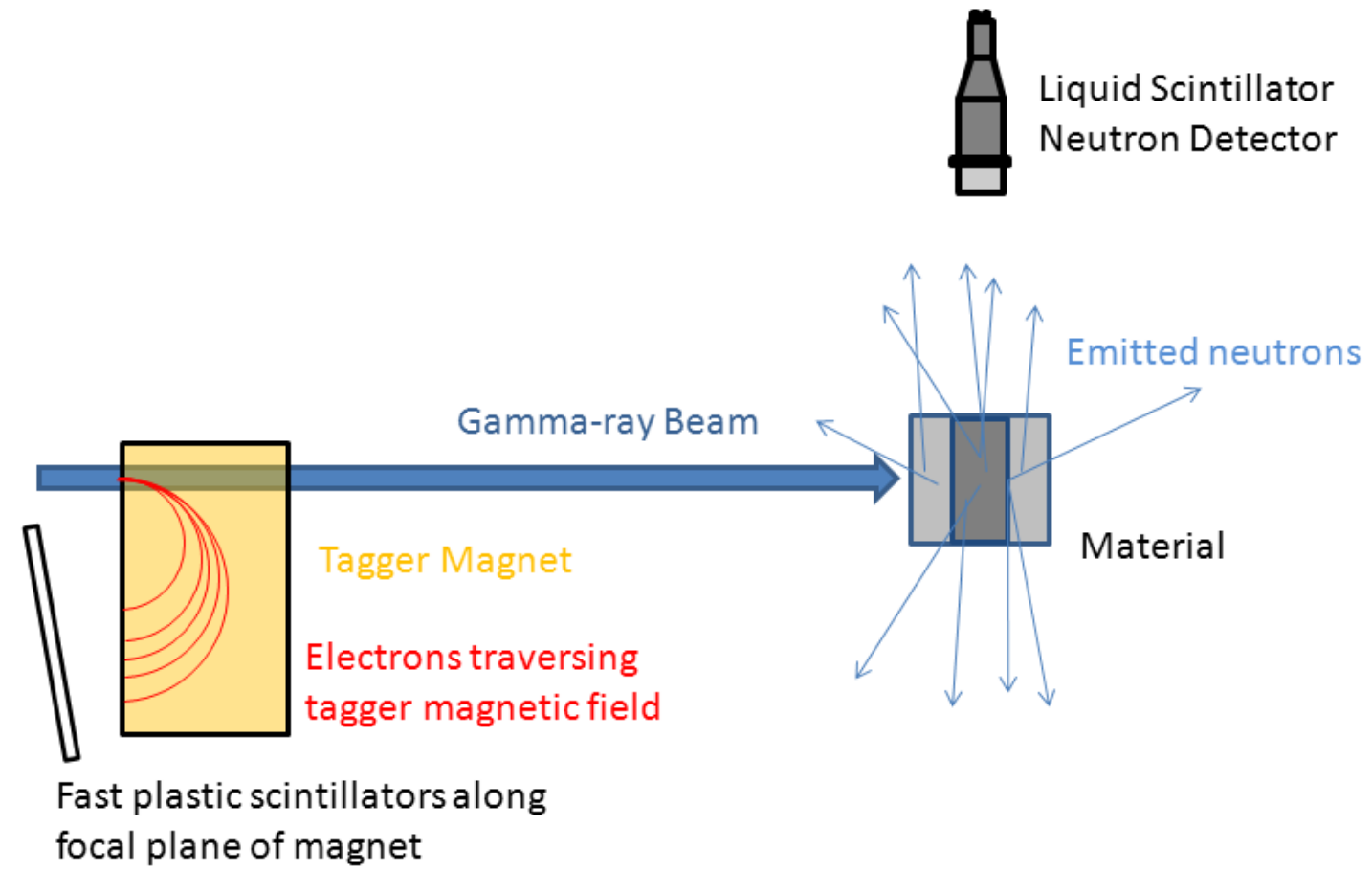

Figure S7. Tagged photon active interrogation scenario assumed for PNNL computational modeling study of material discrimination based on photoneutron production. 


\section{Tagger $\Delta \mathrm{E}=500.0 \mathrm{keV} / \mathrm{chan}$}

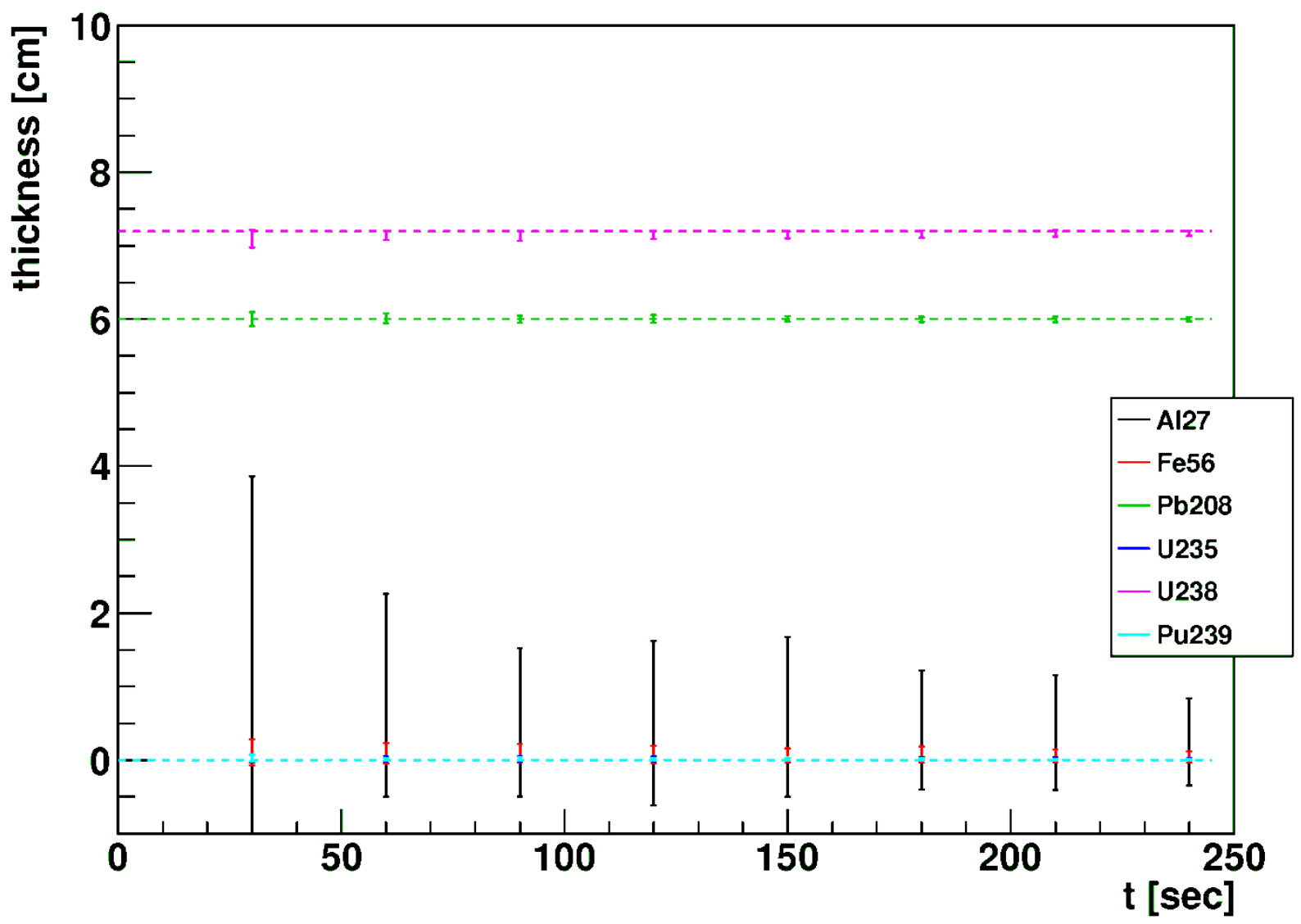

Figure S8. Simulated, reconstructed target material thicknesses for the tagged-photon measurement scenario displayed in Figure S7, as a function of dwell time. Dotted lines indicate "ground truth" thickness of U-238 (pink) and Pb-208 (green) in a sandwich target consisting of slabs of these two materials. The reconstruction algorithm attempts to account for the measured neutron yield as a function of tagged photon energy in terms of a linear superposition of yield shapes drawn from the nuclide set indicated in the legend. The points with error bars indicate the reconstructed thicknesses. An 18-MeV endpoint bremsstrahlung beam is assumed, generated by a $20 \mathrm{nA}$ electron beam incident on a 150-micron Al foil. The neutron detector is assumed to have an intrinsic, energy-independent efficiency of $25 \%$ for fast neutrons, and is positioned $0.5 \mathrm{~m}$ from the target. The dotted blue curve at zero thickness indicates that none of the other library materials is included in the target sandwich. Reasonable null results are obtained for all library materials not present in the target sandwich, except for Al. A weakness of the reconstruction algorithm as currently formulated is that it is susceptible to indicating a spurious Al-27 content, as in this example.

The discussion of photon-tagging in the main body of the report concludes with a design assessment for beamline infrastructure required to implement photon-tagging at an electron accelerator facility, with particular emphasis on energies spanning the $\sim 6 \mathrm{MeV}$ to $20 \mathrm{MeV}$ response region investigated in the photoneutron modeling. Several aspects of the pulseprocessing and data-collection electronics required for high-precision photon tagging work are discussed in detail. 


\section{Acronyms and Abbreviations}

CW

D-D

D-T

DU

API

APT

AI

FOM

HEU

LDRD

MDA

MUF

NORM

PNNL

$\mathrm{Pu}$

RDD

RDMD

RMS

ROC

SNM

sr

TOF

VHEU continuous wave

Deuteron-deuteron (reaction)

Deuteron-triton (reaction)

depleted uranium

associated particle imaging

associated particle tagging

active interrogation

figure of merit

highly enriched uranium

laboratory-directed research and development

minimum detectable activity

material unaccounted for

naturally occurring radioactive material

Pacific Northwest National Laboratory

plutonium

radiological dispersal device

Radiation Detection Materials Discovery

root mean square

receiver operating characteristic

special nuclear material

steradian

time of flight

very highly enriched uranium 


\section{Contents}

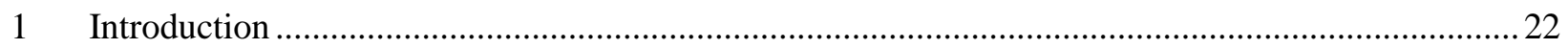

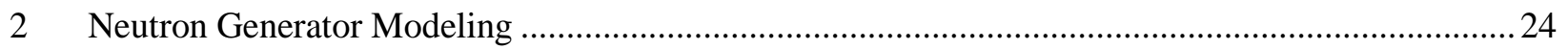

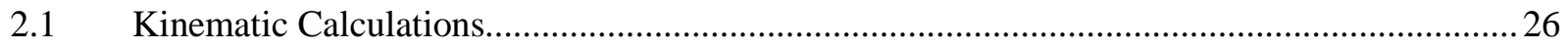

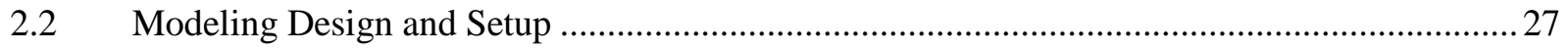

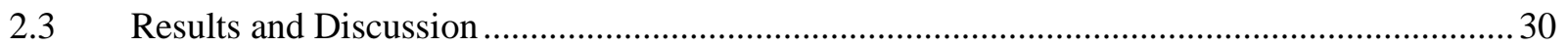

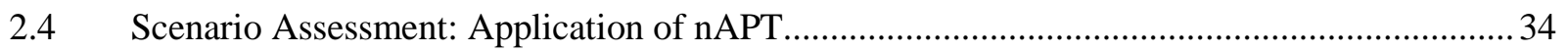

2.5 Neutron Generator Modeling: Conclusions and Future Work................................................. 36

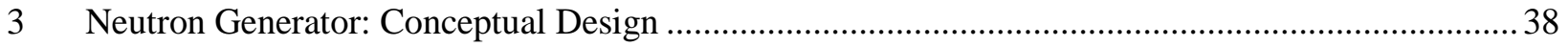

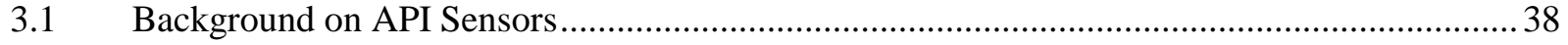

3.2 Conceptual Layout of the PNNL API ENG ……...................................................................... 39

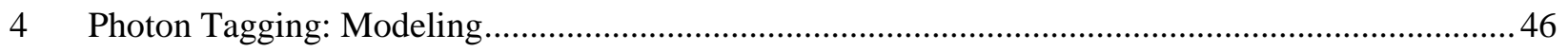

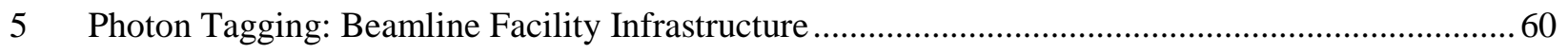

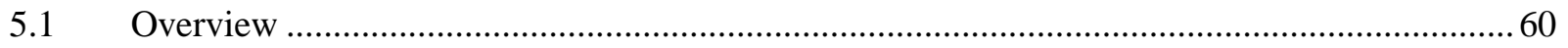

5.2 Measurement of absolute photonuclear cross sections with the photon tagging technique ....... 60

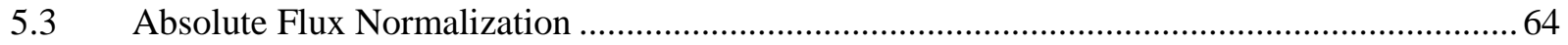

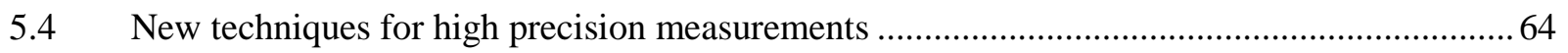

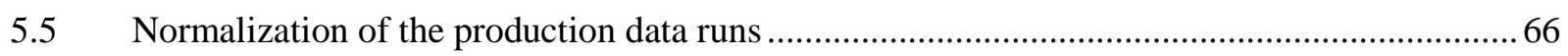

5.6 Relative photon flux calibration with pair production......................................................... 71

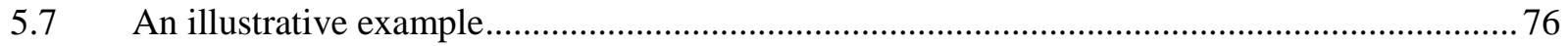

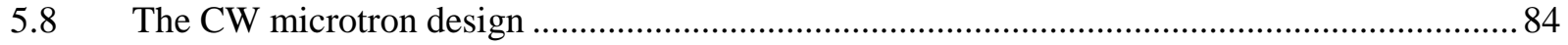

5.9 Tagging Spectrometer: Ion-Optical Notional Design........................................................... 89

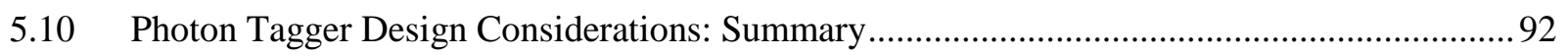

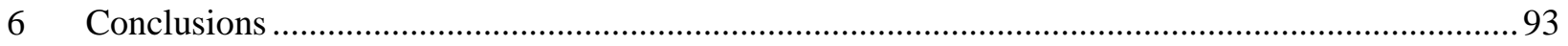

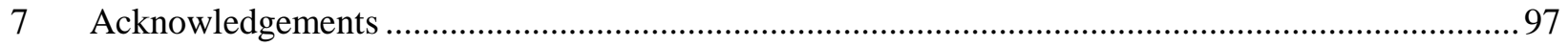

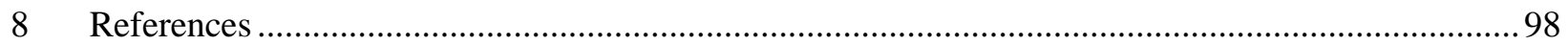




\section{Figures}

Figure 2-1. Conceptual design of a solenoidal magnetic spectrometer (image reproduced from Wuosmaa

[2007]).

Figure 2-2. Dependence of solenoid magnetic spectrometer parameters on field strength. 27

Figure 2-3. Schematic drawing of nAPT magnetic spectrometer concept (not to scale). 28

Figure 2-4. Rendering of the alpha particle trajectory (in blue) around the axis of the magnetic spectrometer, eventually striking the Si detector array (green highlighted dashed lines). The red and green line segments at oblique angles to the cylinder axis are coordinate axes, drawn in perspective.

Figure 2-5. Neutron kinetic energy distribution as a function of emission angle relative to magnetic spectrometer cylinder axis. Variation of the neutron energy with angle is calculated according to relativistic kinematics in the two-body $D+T$ reaction.

Figure 2-6. Calculated energy of the alpha particle as a function of the alpha-detector pixel position along the magnetic spectrometer axis. The energy resolution as a function of position is also shown.

Figure 2-7. Alpha particle energy spectra from each Si detector, as a function of axial position from the target. The increase in dispersion of deposited energies at smaller positions (i.e. larger emission angles) is due to the distribution of alpha-particle energy losses in the target over the angular acceptance of the corresponding detector pixel. The pixel size for the spectra shown is $3 \mathrm{~mm}$.

Figure 2-8. Simulation results for reconstructed neutron emission angle and associated angular resolution. Note that $2^{\circ}$ angular resolution or better can achieved over approximately $65^{\circ}$ of neutron polar angle.

Figure 2-9. Simulated example application of tagged neutrons for cargo scanning. The three objects are color enhanced for improved visualization. Black dots correspond to scattering

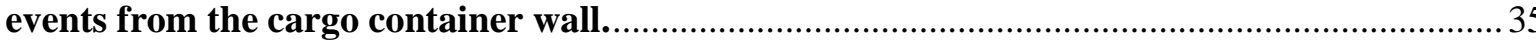

Figure 3-1 Conceptual cut-away drawing of a proposed layout for the PNNL API ENG.

Figure 3-2 External view of a conceptual layout for an API ENG using a helical alpha trajectory sensor.

Figure 3-3 Cutaway view of a conceptual layout for an API ENG using a helical alpha trajectory sensor, showing the neutron tube.

Figure 3-4 Cutaway view of a conceptual layout for an API ENG using a helical alpha trajectory sensor, viewing inside the neutron tube to see alpha-particle sensor.

Figure 3-5 Cutaway view of internal components in a conceptual layout for an API ENG using a helical alpha trajectory sensor.

Figure 4-1: Photoneutron yield versus incident gamma-ray energy for six materials which represent a wide mass range. 
Figure 4-2: Tagged photon rate for $100 \mathrm{keV}$ wide tagger channels assuming the beam properties described in the text..

Figure 4-3: Schematic of simulated photon-tagging scenario. The figure omits generation of the photon beam via bremsstrahlung of the primary electron beam in the radiator target. The blue arrow suggests the mean direction of the bremsstrahlung photon beam, but does not illustrate the angular envelope of the photon beam.

Figure 4-4: Simulated time-of-flight spectrum versus the tagged photon energy, $\mathrm{E}_{\gamma}$, for a Pb interrogation target. Photoneutrons are visible as a broad feature at about $90 \mathrm{~ns}$ in the timing spectrum and at incident photon energies above $9 \mathrm{MeV}$. 50

Figure 4-5: Simulation of the importance of accounting for accidental background in a photon tagging system. The black curve illustrates the apparent photoneutron yield (black curve) reconstructed from bombardment of a $\mathrm{Pb}$ target without applying an appropriate background subtraction. The actual neutron yield distribution supplied as input to the simulation is plotted in red.....

Figure 4-6: Reconstructed neutron yield on $\mathrm{Pb}$ after applying the out-of-time background subtraction. Note that in this simulation, the beam end point energy was $15 \mathrm{MeV}$.

Figure 4-7: Comparison of the two-fold differential photoneutron cross section for Fe-56 (left) and U-235 (right). Data are from [Chadwick 2006].................................................................................. 52

Figure 4-8: $E_{n}$ versus $E y$ photoneutron yield distributions for ${ }^{208} \mathrm{~Pb}$. On the left the tabulated cross section data are interpolated using the Delaunay method. On the right, the unit-base transform method is used. The yields are plotted in arbitrary units; the purpose of this comparison is to illustrate the qualitative difference in structure of the yields for the two interpolation methods... 53

Figure 4-9: Comparison of ENDF evaluated photoneutron yield data with the yield from [Dietrich 1988]. In order to make this comparison, the ENDF cross section tabulations have been integrated over neutron angle from the $60^{\circ}$ data assuming isotropy.

Figure 4-10: Results of the target composition reconstruction simulation using the shape of the photoneutron cross section as a function of gamma-ray energy and integrating the neutrons from 2 $\mathrm{MeV}$ and up, with $500 \mathrm{keV}$ per tagger channel. The top figure uses the cross section shape as a function of gamma-ray energy (1D approach) only, whereas the bottom figure includes the variation of the cross section with outgoing neutron energy as well (2D approach).

Figure 4-11: Same as Figure 4-10, but assuming a tagged-photon energy width per channel of $200 \mathrm{keV}$ rather than $500 \mathrm{keV}$.

Figure 5-1. Schematic layout of a notional pair spectrometer for continuous relative flux normalization of tagged photons. Each arm consists of eight continuous plastic scintillator hodoscopes in each row. The tagged photon beam enters the spectrometer from the lower-left. Pair production yields secondary electrons and positrons which are split into spatially-separated beams by a magnetic field.

Figure 5-2. Distribution of time differences for events reconstructed for tagger and photon detector (in this case a lead glass detector) showing the \pm 4 .5-ns timing window for coincidence events. Data are from the Jefferson Laboratory PrimEx experiment. 
Figure 5-3. Time spectrum of hits reconstructed for a single T-counter. Data from the Jefferson Laboratory PrimEx experiment.

Figure 5-4. A close up of part of the previous plot illustrating the drop off of the number of hits due to the LIFO limit.

Figure 5-5. Timing spectrum of hits reconstructed for a single T-counter. These data were taken with clock triggers. Data from the Jefferson Laboratory PrimEx experiment.

Figure 5-6. Distribution of time differences for events reconstructed in the tagger and pair spectrometer

Figure 5-7. Measured $R_{\text {rel }}^{i}$ for T-counter \#3 as a function of nominal electron beam current. Data from

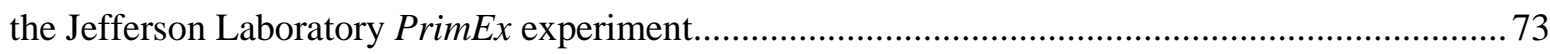

Figure 5-8. The percent deviation from the mean for tagging ratio measurements made at different........74

Figure 5-9. Run-to-run stability of $R_{\text {rel }}{ }^{\text {combined }}$ - relative tagging ratio combined for eleven T-counters -carbon target. Data from the Jefferson Laboratory PrimEx experiment. ...................................... 75

Figure 5-10. Ratio of neutrons emitted back-to-back versus parallel in the fission model described in the

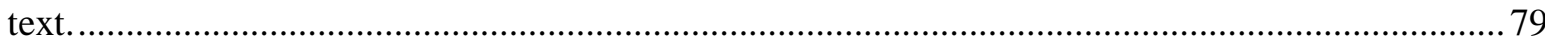

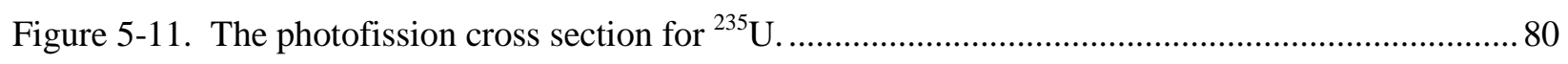

Figure 5-12. The direct two neutron emission cross section for ${ }^{235} \mathrm{U}$. ................................................. 81

Figure 5-13. Calculated bremsstrahlung photon yield for a $7 \mathrm{MeV}$ electron beam................................. 82

Figure 5-14. A time of flight spectrum measured using the IAC High Repetition Rate Linac. Solid ....... 83

Figure 5-15. Cross section of a linac RF cavity with multiple cells. ..................................................... 85

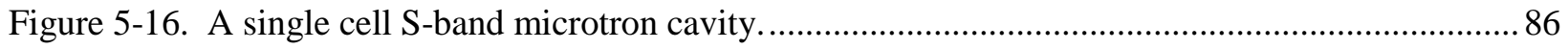

Figure 5-17. Schematic layout of a general racetrack microtron. Here the electron beam is initially injected from right to the left and then it passes through the linac structure. Because the beam at this point does not have enough momentum to make it past the linac structure due to its small bending radius, it is then bent 180 degrees at the left dipole and passes through the linac structure again. All subsequent orbits are then counterclockwise.

Figure 5-18. End magnets from a $50 \mathrm{MeV}$ microtron

Figure 5-19. Overhead view of tagging spectrometer dipole, example electron trajectories, and focal plane position. Distinct bunches of electron trajectories correspond to different momenta. The locations of the narrow waists in the bunches following traversal of the dipole magnet define the focal "plane" (or focal surface). Note that the electron trajectories within the dipole are not plotted accurately in this figure; only the trajectories before and after the dipole correspond to the model of physical paths.

Figure 5-20. Calculated energy resolution of the notional photon tagging spectrometer as a function of post-bremsstrahlung electron energy. The corresponding tagged-photon energy at 20-MeV endpoint energy runs from $6 \mathrm{MeV}$ (right side of plot, electron energy = $14 \mathrm{MeV}$ ) to $18 \mathrm{MeV}$ (left side of plot, electron energy $=2 \mathrm{MeV}$ ). The curves correspond to different assumptions about the width of the focal plane detector. The "intrinsic" case represents the limit of the resolution for zero detector 
width. For $1 \mathrm{~mm}, 2 \mathrm{~mm}$, and $10 \mathrm{~mm}$ detector widths, the 71-cm focal plane extent in the $y$-axis direction (see axes in Figure 5-18) is spanned by 710, 350, and 71 detectors, respectively.

Figure 6-1. Notional design for prototype position-sensitive Si detector array, taken from [Wuosmaa 2007]...... 


\section{Introduction}

The primary objective of the Associated Particle Tagging (APT) in Magnetic Spectrometers project at Pacific Northwest National Laboratory (PNNL) is to explore the utility of magnetic (i.e. ion-optical) spectroscopy techniques as an enabling technology for producing advanced and/or unique capability in tagged neutron and tagged photon active interrogation (AI) sources. The adjective "tagged" in the description of this class of sources denotes detection of an associated reaction product involved in generation of the probe-beam particle (neutron or photon) of interest. Measurement of kinematic (energy and direction) and timing properties of the associated particle can aid in defining the energy and spatial properties of the probe beam on a particle-by-particle basis. As applied to AI, the tagging technique exploits this kinematic information to map the energy dependence and/or spatial dependence of the inspected item's response to the interrogating beam. Features of this interrogation response useful for detecting special nuclear material (SNM) are of particular interest within the context of this NA-22 funded project. The research scope of the APT project addresses both neutron and photon AI sources. We have examined two principle concepts in a computational and modeling-based feasibility study of ion-optical detection and spectrometry approaches for advanced tagged sources:

1. Application of the solenoidal spectrometer to development of a compact, large-emittance associated particle imaging (API) neutron source. The associated particle in this case is the alpha particle in the deuterium + tritium (DT) reaction relevant to fast (14.1 MeV) neutron production, $\mathrm{D}+\mathrm{T} \rightarrow \alpha+\mathrm{n}$. (Alternatively, one can detect the outgoing deuteron in the deuterium + deuterium (DD) reaction, relevant to production of neutrons at energies of about 2.2 MeV.) Tagging the neutron by detection of the associated alpha defines the direction and timing of the outgoing neutron. Use of the neutron tagging technique in API neutron-generator systems is not novel. The current project addresses a novel means of detecting and momentum-analyzing the alpha particle which offers potentially substantial improvements, relative to existing methods, in the tagged fraction of outgoing neutrons and robustness of the neutron generator.

2. Application of bremsstrahlung photon tagging to photonuclear detection of SNM. The associated particle detected is the post-bremsstrahlung electron following generation of a bremsstrahlung photon in a thin target. The main goal is to reconstruct the tagged photon's energy from the difference in the (known) primary electron beam energy and the (measured) final-state electron energy. Tagged photon beams have been applied to cross section measurements in experimental nuclear physics for several decades. The present project investigates the potential advantages (over conventional broad-beam bremsstrahlung sources) of the tagged-photon energy information for photonuclear detection of fissile materials.

The motivation for performing this feasibility assessment differs somewhat for the two source types. Detection of the alpha-particles produced in the DT reaction has already been exploited in developing the API technique, and electronic neutron generators (ENGs) implementing alphaparticle detection are the subject of ongoing research in the national laboratory community. Application of wide angular-acceptance, ion-optical spectrometry to alpha detection in a nextgeneration API generator may offer significantly larger tagging fractions (i.e. the portion of the outgoing neutron angular distribution tagged by the detected alpha) and enhanced physical 
robustness relative to current designs. In contrast, tagged-bremsstrahlung photon sources are not currently deployed for AI applications. The current feasibility study aims to identify and quantify the utility of photonuclear AI methods for exploiting event-by-event photon energy definition of order a few percent in $\Delta \mathrm{E} / \mathrm{E}$. We focus in particular on tagged-photon approaches to stimulating emission of fast neutrons from fissile materials, with the goal of mapping the energy-dependent neutron yield as a function of photon energy, neutron energy, or both, as a signature of the presence of fissile materials in the interrogated sample.

In broad outline, the research methodology involves a similar task structure for both tagged neutron and tagged photon source types:

1. Perform radiation transport modeling, supporting analytical modeling, and scenario analysis as appropriate to bound the anticipated performance benefits arising from successful implementation of tagging techniques;

2. Leverage "real-world" operational expertise to formulate elements of a conceptual design of instrumentation implementing the tagged-particle approach, and to quantify the technical feasibility of achieving a specified level of spectrometer system performance.

Note that the technical feasibility assessment arising from task (2) will in general depend implicitly upon the investment assumed for a full-scale engineering design (as opposed to conceptual design) R\&D effort. While the present project does not aim to attach dollar values to a specified system performance, the initial feasibility assessments documented in this report will provide guidance for future, more detailed evaluations of component technical readiness and implementation costs. We aim to summarize the performance-benefit and conceptual design assessments at a level of detail sufficient to provide an informed basis for future decisions on the proper role (if any) of magnetic spectrometry in tagged-particle active interrogation sources.

The report is organized by source type as follows. Sections 2 and 3 detail, respectively, modeling and conceptual design considerations for a next-generation API neutron generator incorporating magnetic spectrometer-based alpha particle detection. This portion of the work reflects a collaborative effort between the PNNL project team and Dr. David Chichester of Idaho National Laboratory (INL). Section 2 describes radiation-transport modeling of a notional neutron generator incorporating a magnetic spectrometer (with a pixelated silicon detector array) for alpha-particle detection. Section 3 provides historical and operational context for the API neutron-generator technique and outlines elements of a conceptual design for implementing the novel alpha-particle detection method. Sections 4 and 5 describe, respectively, application modeling and conceptual design for the tagged-photon technique. The PNNL team has collaborated with Dr. Daniel Dale and colleagues at the Idaho State University (ISU) and the Idaho Accelerator Center (IAC) in this aspect of the work. Section 4 describes radiation transport modeling and scenario assessments for application of the tagged-photon technique to photonuclear fast-neutron response yield discrimination of fissile from non-fissile materials. Section 5 documents a variety of conceptual design and application issues for installation of a tagging system at an existing accelerator facility. Although the present project emphasizes AI, section 5 also addresses the potential use of a dedicated tagging facility for high-precision nuclear cross-section measurements relevant to future threat reduction modeling assessments. 


\section{Neutron Generator Modeling}

Neutron generators are used in a variety of applications, including oil well logging, nuclear physics, and national security. It is often beneficial to "tag" the neutrons such that the correlation of time and direction of the neutron emission can be exploited for spatial imaging of the inspected item (via associated particle imaging, or API) and for background reduction [Chichester 2005]. Current systems tag the charged particle emitted from the deuteron-triton (DT) or deuteron-deuteron (D-D) reaction using a relatively thin inorganic scintillator coupled to a position-sensitive photodetector. These systems, in general, are relatively fragile and can only reconstruct approximately 1 steradian of emitted neutrons. Alternative tagging methods that increase the angular emittance of the tagged neutron beam will in general permit more efficient use of the neutron yield, thus increasing the effective generator lifespan. Methods that avoid the use of a thin window and an externally-mounted photosensor can potentially improve generator robustness against mechanical shock.

One candidate technique that achieves both of these objectives is to employ a solenoidal magnetic spectrometer for detection of the outgoing $\alpha$-particle in the D-T reaction, such as the one developed by Wuosmaa [2007]. Figure 2-1 displays a schematic of a solenoidal spectrometer for general application to cross section measurements in charged-particle reaction physics. In this technique, a charged particle detector and beam target are placed inside the uniform magnetic field of a solenoid. Assuming an ion beam of specified energy incident upon a thin target (in which energy straggling and multiple scattering can be neglected), the motion of the reaction products are completely described by two-body kinematics, combined with charged particle transport within a uniform magnetic field. These properties of the device can be exploited to measure the outgoing neutron kinematics (direction and energy) on an event-by-event basis, as described below.

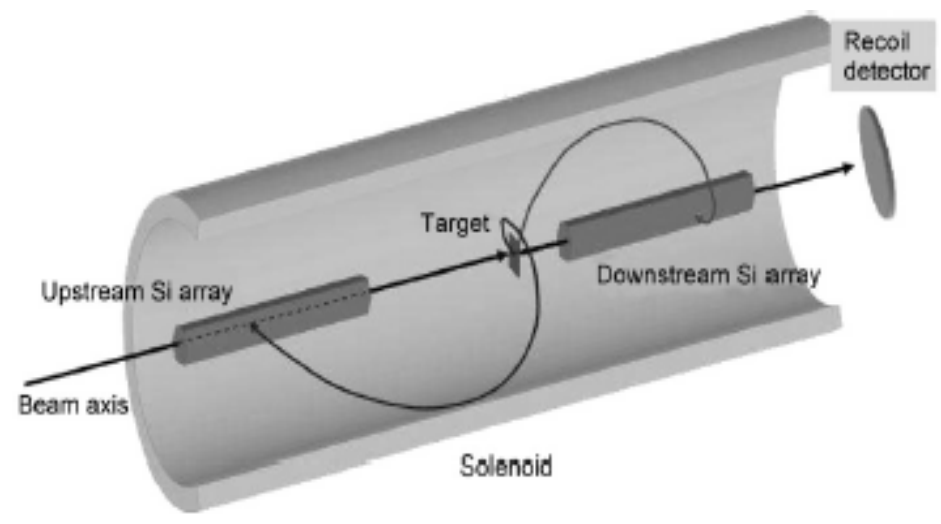

Figure 2-1. Conceptual design of a solenoidal magnetic spectrometer (image reproduced from Wuosmaa [2007]).

Reconstruction of the charged-particle momentum vector at the reaction vertex is achieved by exploiting knowledge of the (energy-independent) time for the particle to execute helical motion in the solenoidal magnetic field and return to the longitudinal axis. A measurement of the 
longitudinal position at which the particle returns to the axis, in tandem with the known period for the circular component of the helical motion in the magnetic field, yields the longitudinal component of the charged-particle momentum. A measurement of the energy deposited by the charged particle in the detector placed along the spectrometer axis determines the magnitude of the particle's (total) momentum. These two pieces of momentum information then determine the polar angle of emission at the target. The azimuthal emission direction is reconstructed by measuring the azimuthal position of particle arrival at the spectrometer axis. This requires position sensitivity in the azimuthal direction, which can be achieved with e.g. a hollow annular or multi-faced rectangular-tube detector array. This fully specifies the charged-particle momentum vector at the target exit. For the purpose of tagging neutrons, one then exploits twobody kinematics (in which, for energies relevant to active interrogation, the reaction products are emitted essentially back-to-back in the laboratory) to determine the outgoing neutron momentum vector at the reaction vertex from the reconstructed $\alpha$-particle momentum.

Thus, it is possible in principle to reconstruct the direction and energy of neutron emissions produced by such a device. (As in standard API, the arrival time of the $\alpha$-particle at the spectrometer's charged-particle detector array furnishes a timing fiducial for production of the tagged neutron.) In practice, energy-loss straggling of the incident beam and multiple scattering of the outgoing $\alpha$ in the finite-thickness target will tend to limit the angular and energy resolution of the neutron momentum reconstruction. Additional contributions to the reconstruction resolution will arise from the finite position resolution of the charged-particle detector array (both in the longitudinal and azimuthal directions) and from the finite resolution in the chargedparticle energy-deposition measurement. The primary purpose of the modeling studies discussed in this section is to quantify the neutron tagging resolution (i.e. the precision in determining the outgoing neutron's direction and energy) and the neutron tagging efficiency (i.e. the fraction of the outgoing neutron flux tagged by detection of the accompanying $\alpha$-particle product of the D-T reaction). Knowledge of these quantities for a notional spectrometer design will aid in informing an assessment of the feasibility and the value-added (relative to conventional API neutron generators) of using such a system in neutron associated particle tagging (nAPT).

The use of a large-bore magnetic solenoid with a magnetic field strength of order 1-10 $\mathrm{T}$ has several potential advantages over traditional neutron generators with a coated inorganic scintillator. Particles emitted from the target will follow a helical trajectory, and after a single orbit return to the axis where we place a Si detector array. In neutron tagging, where one wants to tag the neutron from the D-T reaction, the use of the magnetic spectrometer design leads to a significant decrease in background processes that complicate the tagging process. Only particles with appropriate momentum and charge (in this case, alpha particles) will be directed toward the Si detector array. Other particles, such as electrons and scattered beam ions, can be readily discriminated from the alpha particles due to their very different energies and trajectories. The use of a wide-angle spectrometer increases the effective solid angle for detection of outgoing alpha-particles relative to a conventional API scheme (in which the alpha detector views the target from fixed standoff at the boundary of an essentially field-free region). An upper limit for the fraction of neutrons usefully tagged by the spectrometer method is 0.5 , as opposed to the conventional method (with 1 steradian solid angle acceptance), 0.08. With a larger fraction of the outgoing neutron flux tagged, the generator can be placed closer to the inspected item without sacrificing angular coverage. This in turn improves geometrical efficiency of the 
neutron interrogation and extends effective generator ion-target life. Finally, the neutron generator concept incorporates all of the alpha-particle detectors in the interior of the generator housing, providing a potentially substantial improvement in robustness relative to current API neutron generator designs which incorporate externally-mounted alpha sensors viewing the target through a port in the generator housing.

\subsection{Kinematic Calculations}

In the D-T reaction, an alpha particle is emitted according to the conservation of energy and momentum. The reaction is isotropic, whether the reaction is at thermal energies, or at the slightly accelerated $\mathrm{T}$ kinetic energy of $100 \mathrm{keV}$. While D-D reactions should also be noted as a potential reaction in neutron generators, the D-T reaction is much more frequent. Much of the details of charged particle transport within a solenoid is described elsewhere [Wuosmaa 2007], however we summarize some of the formulation here. The cyclotron period is the time required for the particle to move in its helical motion back to the axis. This period, $\mathrm{T}_{\mathrm{cyc}}$, is given by

$$
T_{c y c}=\frac{2 \pi}{B} \frac{m}{q e},
$$

where $\mathrm{B}$ is the magnetic field strength, $\mathrm{m}$ is the mass of the alpha particle, and qe is the charge of the particle. A significant advantage of the magnetic spectrometer solenoid design is that the cyclotron period is independent of the particle energy or the emission angle from the foil. Using this value removes some experimental uncertainty from trying to estimate the particle transport time by other means. Since particles will always return to the axis, the maximum bore radius

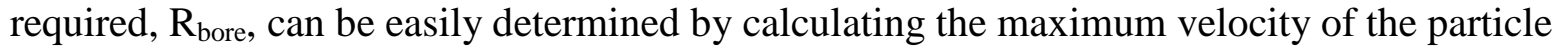
perpendicular to the axis of the solenoid.

$$
\mathrm{R}_{\text {bore }}=\frac{\mathrm{T}_{\text {cyc }}}{2 \mathrm{v}_{\text {perp, } \max }} .
$$

The maximum axial distance $(\mathrm{z})$ the particle will travel prior to crossing the axis can calculated by noting the cyclotron period and the maximum velocity of the particle parallel to the magnetic field,

$$
\mathrm{z}=\mathrm{v}_{\text {para }} \mathrm{T}_{\text {сус }}
$$

Thus, a number of important parameters of the solenoid magnetic spectrometer are determined primarily by the magnetic field strength. Figure 2-2 depicts these three parameters as a function of magnetic field strength for the alpha particle in the D-T reaction (100 keV kinetic energy associated with the tritium particle). All of the properties are inversely related for magnetic field strength. Thus, using high fields allows one to construct a more compact system, although there a practical limitations to developing the compact magnetic spectrometer. If the relatively compact system (e.g., less than $1 \mathrm{~m}^{3}$ ) is desired, the minimum field strength should be 2T, in order to limit the maximum axial distance required for the system.

By measuring the position, energy and time of the alpha particle strike on the Si detector array, information about the associated neutron can be determined. It is then possible to vector collimate the neutron (i.e., restrict the angular envelope of the outgoing neutron and reconstruct its outgoing direction on the basis of the detected alpha particle) such that the angular resolution 
of reconstructed neutron angle can be on the order of $1^{\circ}$ or less, depending on the position resolution of the Si array. So long as the particles do not strike the solenoid walls or exit the end of the solenoid prior to crossing the axis, a $2 \pi$ azimuthal angle range is easily feasible and therefore a neutron tagging system with an increased acceptable angle approaching $2 \pi$ steradians is feasible with the magnetic spectrometer design.

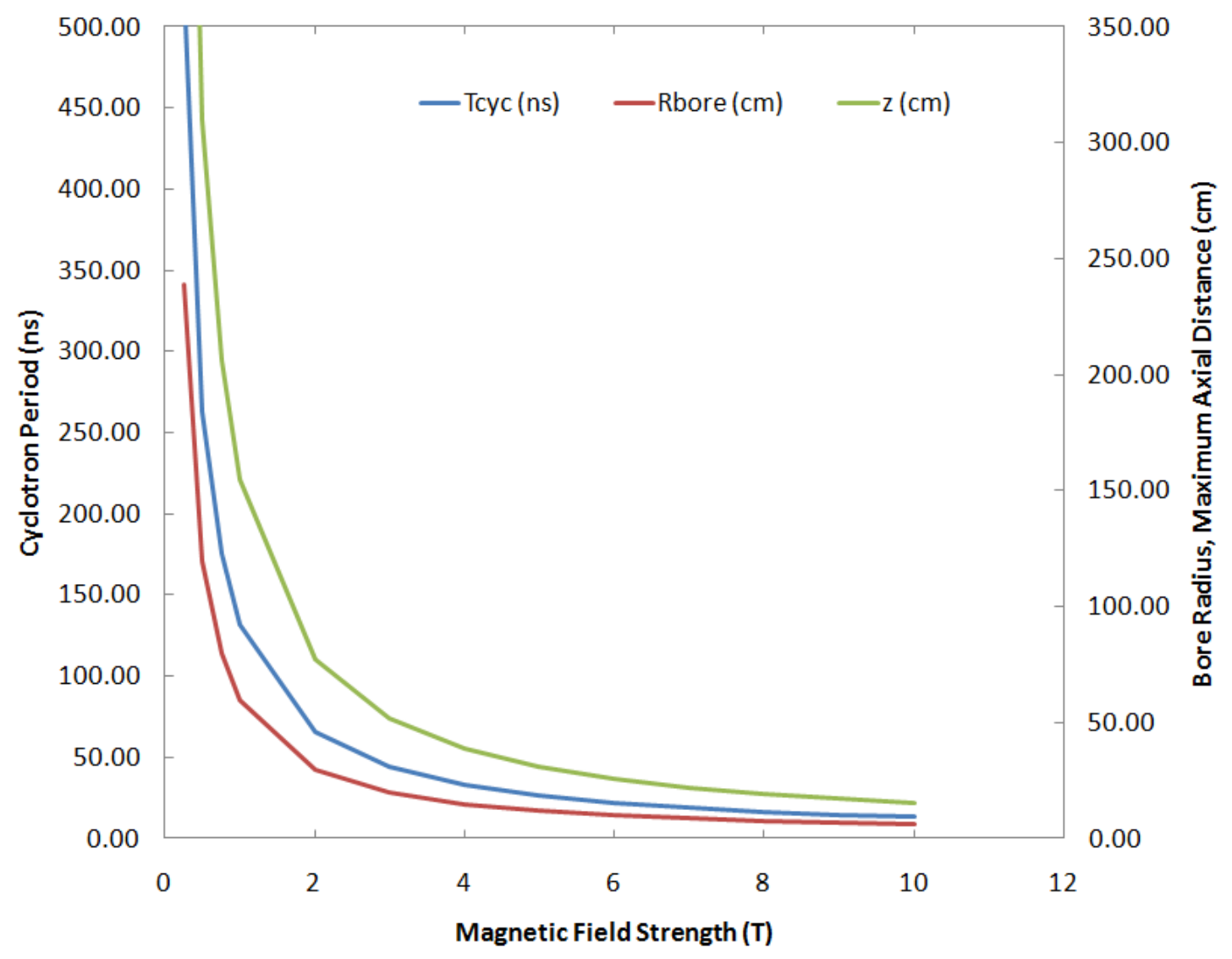

Figure 2-2. Dependence of solenoid magnetic spectrometer parameters on field strength.

\subsection{Modeling Design and Setup}

We used Geant4 [Agostinelli 2003] (version 9.3) to model the magnetic spectrometer and neutron generator. Figure 2-3 below shows a cut-away sketch of the model. A solenoid consisting of several primary materials ( $\mathrm{Cu}$ for the current-carrying coil, cryogenic fluids and $\mathrm{Al}$ and plastic support materials) was simply and approximately modeled, primarily to assess the impact of scattered radiation on the tagging capabilities. A cylindrical array of solid-state Si detectors was placed on the central axis of the solenoid. The beam is incident along the 
spectrometer axis and impinges upon the target material, assumed to be a $\mathrm{TiH}_{2}$ foil target ${ }^{1}$ approximately 1 um in thickness. This thickness is a rough optimum. A thicker target is desirable from the point of view of increasing neutron yield. However, as the target thickness increases, alpha particle energy loss in the detector becomes too large and the ability to reconstruct the neutron emission angle from the position and energy of the alpha particle will degrade. Only events in which (1) the neutron passes through the "threat object" or "neutron detector," AND (2) the alpha particle is detected in the Si detector, are recorded. Not shown in the figure below is a small $\mathrm{W}$ or Ta shield between the target and Si detector array. The purpose of this shield is to block alpha particles that are emitted at very large angles from striking the Si detector array. These alpha particles are associated with large angle neutrons $\left(>70^{\circ}\right)$ that will not pass through the object to be investigated and will only contribute to system noise and improperly tagged neutrons. The use of a $\mathrm{W}$ or Ta is to be able to properly dissipate heat generated from the large number of alpha particles striking this shield.

\section{Threat Object or} n Detector

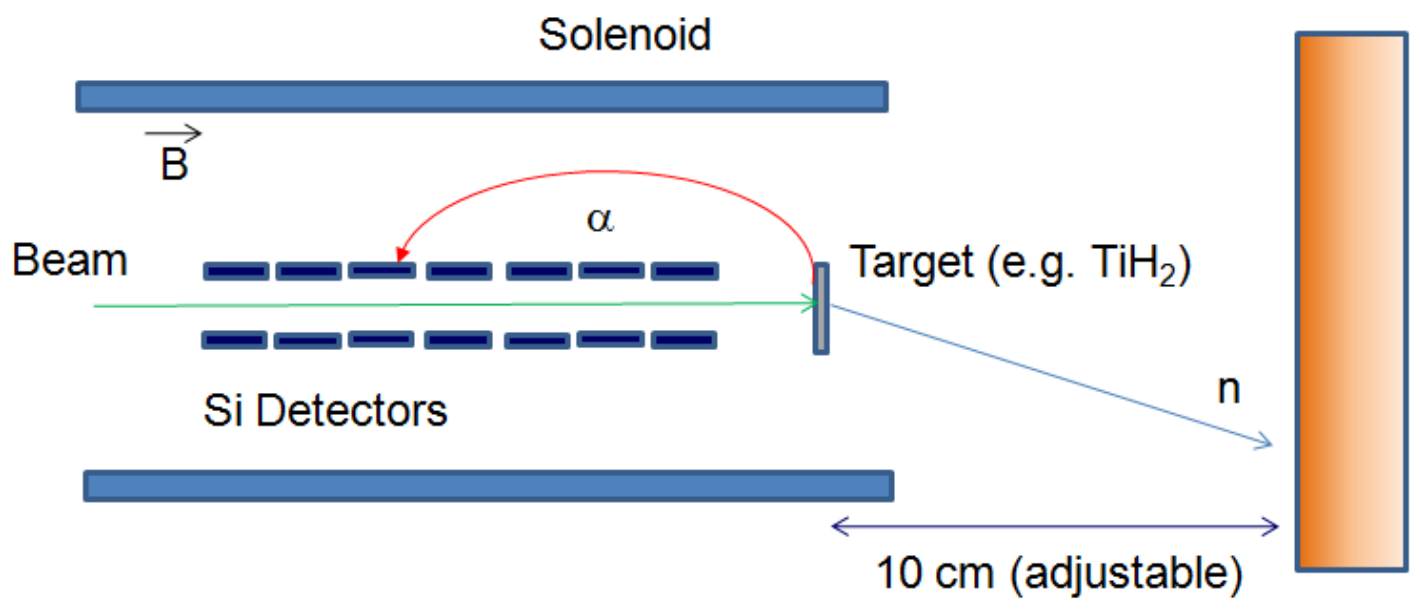

Figure 2-3. Schematic drawing of nAPT magnetic spectrometer concept (not to scale).

Table 1 lists a number of the basic parameters of the nAPT magnetic spectrometer system design that was modeled as part of this study. Several of the parameters used in the models are similar to those currently used in neutron generators and magnetic spectrometers, lending confidence that a near-term solution in developing a prototype system is possible. Figure 2-4 illustrates a typical helical alpha-particle trajectory under the influence of the magnetic field. The alpha

${ }^{1}$ To date we have used $\mathrm{TiH}_{2}$ only, and not $\mathrm{TiD}_{2}$ or $\mathrm{TiT}_{2}$, target materials in the simulation, although the Geant4 framework supports a more detailed description of the isotopic content of user-supplied materials. The final-state particles in the D-T reaction are currently sampled independently of the Geant4 framework's available nuclear reaction physics processes, so the simulated target material is used only for the purposes of evaluating energy straggling and multiple scattering of the charged alpha particle as it exits the target. Because we expect atomic ionization (as opposed to nuclear scattering) processes to dominate the energy loss of alphas, this approximation was deemed reasonable for initial exploratory simulations. 
particle was absorbed within the Si detector array, shown in green highlighted dashed lines within the figure.

Table 1: Parameters for nAPT Magnetic Spectrometer Concept

\begin{tabular}{|l|l|}
\hline Parameter & Value \\
\hline Magnetic Field Strength & Variable (results in this report are based on 5T field) \\
\hline Bore Radius & Up to $45 \mathrm{~cm}$ (variable based on magnetic field) \\
\hline Magnetic Spectrometer Length & $100 \mathrm{~cm}$ \\
\hline Silicon Array Dimensions & $\begin{array}{l}36 \mathrm{~cm} \text { cylindrical shell (inner radius } 0.5 \mathrm{~cm} \text {, outer radium 0.6 cm), pixel } \\
\text { dimensions variable }(1 \text { mm to } 3 \mathrm{~cm})\end{array}$ \\
\hline TiH ${ }_{2}$ dimensions & $\begin{array}{l}2 \times 2 \mathrm{~cm}^{2} ; 2 \mu \mathrm{m} \text { thick (target is larger than beam spot size to accommodate } \\
\text { notional mounting scheme in model) }\end{array}$ \\
\hline Nominal Beam Spot Size & $1 \mathrm{~mm}{ }^{2}$ \\
\hline Gas Pressure & $2 \mathrm{mTorr}$ \\
\hline Beam Energy & $100 \mathrm{keV}$ \\
\hline $\begin{array}{l}\text { Number of D-T reactions } \\
\text { simulated }\end{array}$ & $10^{6} / \mathrm{per}$ simulation \\
\hline
\end{tabular}

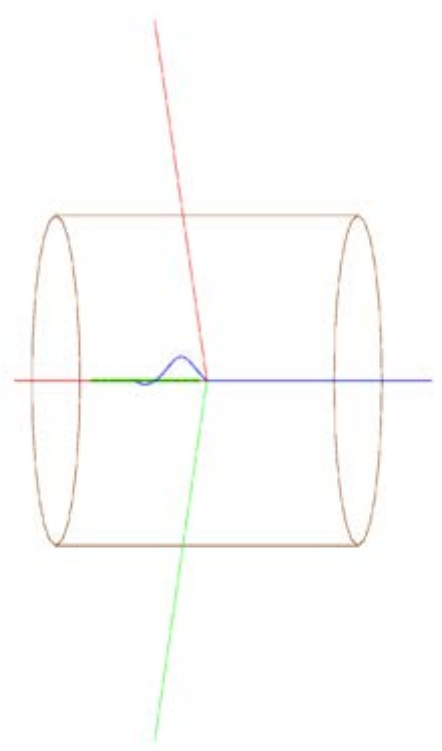

Figure 2-4. Rendering of the alpha particle trajectory (in blue) around the axis of the magnetic spectrometer, eventually striking the Si detector array (green highlighted dashed lines). The red and green line segments at oblique angles to the cylinder axis are coordinate axes, drawn in perspective. 
To simulate the D-T reaction, we generated a mathematical representation of the reaction to generate the alpha particles and neutrons with the correct energies and angular distributions for a $100 \mathrm{keV}$ beam passing down the axis of the magnetic spectrometer and striking the $\mathrm{TiH}_{2}$ target. The simulated "beam spot" of $1 \times 1 \mathrm{~mm}^{2}$, is similar to the beam spot size on current neutron generators. Figure 2-5 below shows the energy distribution of the neutron as a function of the emission angle, and was used to verify the correct operation of our generator by comparing the kinematics for both particles against a relativistic kinematics calculator from TUNL. There was a cutoff around $75^{\circ}$ due to the near edge alpha detector acceptance (which was in agreement with the Si detector placement). Thus, the neutron-alpha generator model was verified to be working properly.

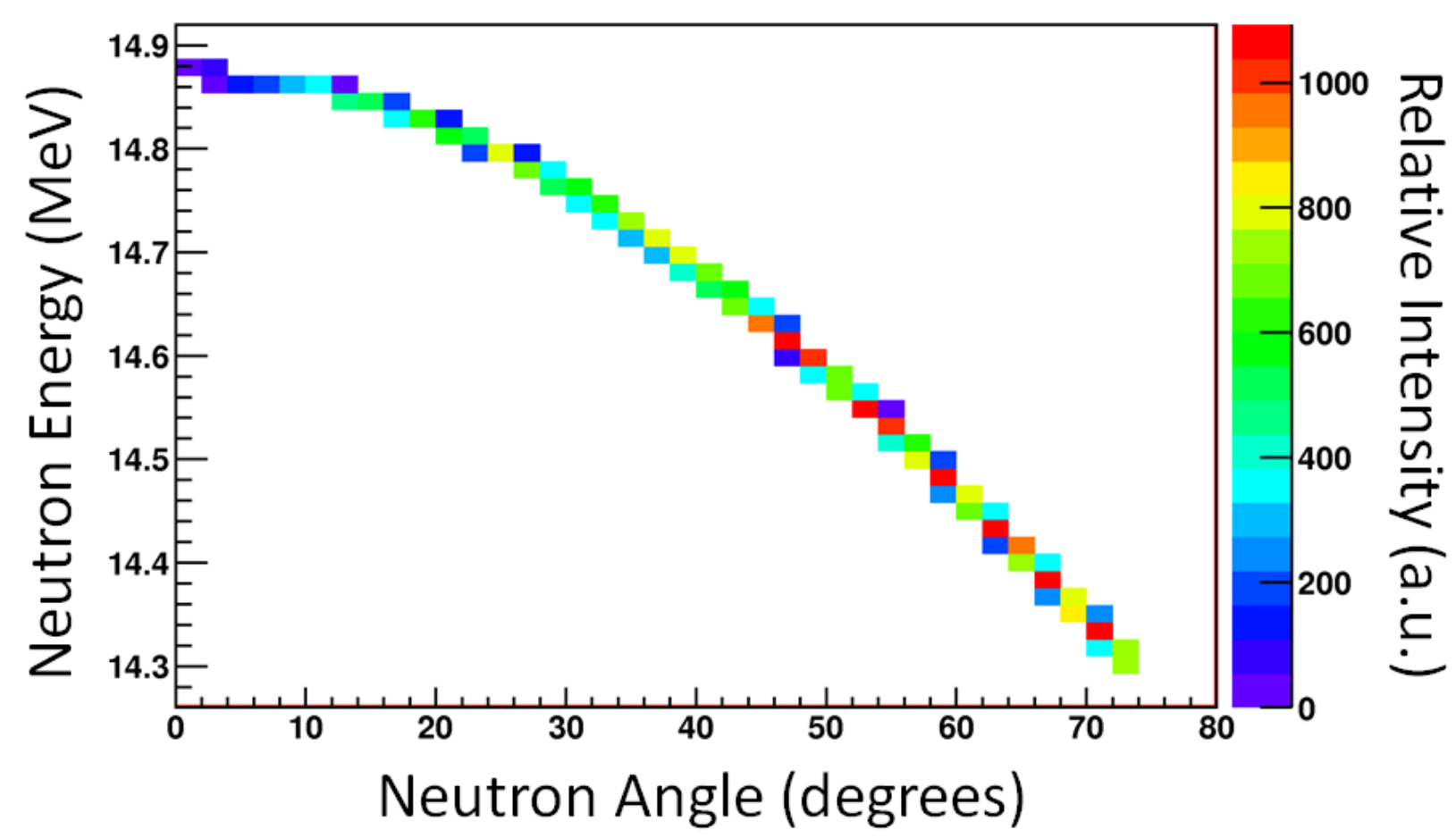

Figure 2-5. Neutron kinetic energy distribution as a function of emission angle relative to magnetic spectrometer cylinder axis. Variation of the neutron energy with angle is calculated according to relativistic kinematics in the two-body $\mathrm{D}+\mathrm{T}$ reaction.

\subsection{Results and Discussion}

Figure 2-6 and Figure 2-7 below show the response of the Si detector array with pixel $3 \mathrm{~mm}$ pixels (the position noted is the center of the pixel). Smaller values of the alpha detector pixel position correspond to positions close to the target and correspond to larger emission angles with respect to the magnetic spectrometer axis. Because of this position-angle correspondence, alpha particles detected at these pixel positions lose a larger amount of energy in the target on average than alpha particles detected in pixels at larger positions. Also, a small change in emission angle for these alpha particles results in a correspondingly larger change in emission energy and therefore a broader deposited energy distribution in the Si detector. At larger distances (above 15 
$\mathrm{cm}$ ), the energy resolution and energy deposited remain relatively constant over the angular acceptance of the pixel. The modeled energy dispersion is approximately $25 \mathrm{keV}$ at this pixelposition limit for 3-mm pixel width, somewhat larger than the assumed energy resolution of 10 $\mathrm{keV}$ for the detector array itself. At larger emission angles, i.e. smaller pixel position, the width of the deposited energy distribution is roughly $500 \mathrm{keV}$, or an order of magnitude larger than the assumed resolution of the energy deposition measurement. Thus in the large emission angle limit, we anticipate that a relatively modest Si energy resolution can yield a meaningful constraint on the alpha emission angle, supplementing the information available from the pixel position. Alpha particle straggling and multiple scattering in the target, and variations in energy loss in the magnetic spectrometer gas ( 2 mTorr), contribute to the degradation in simulated energy resolution.

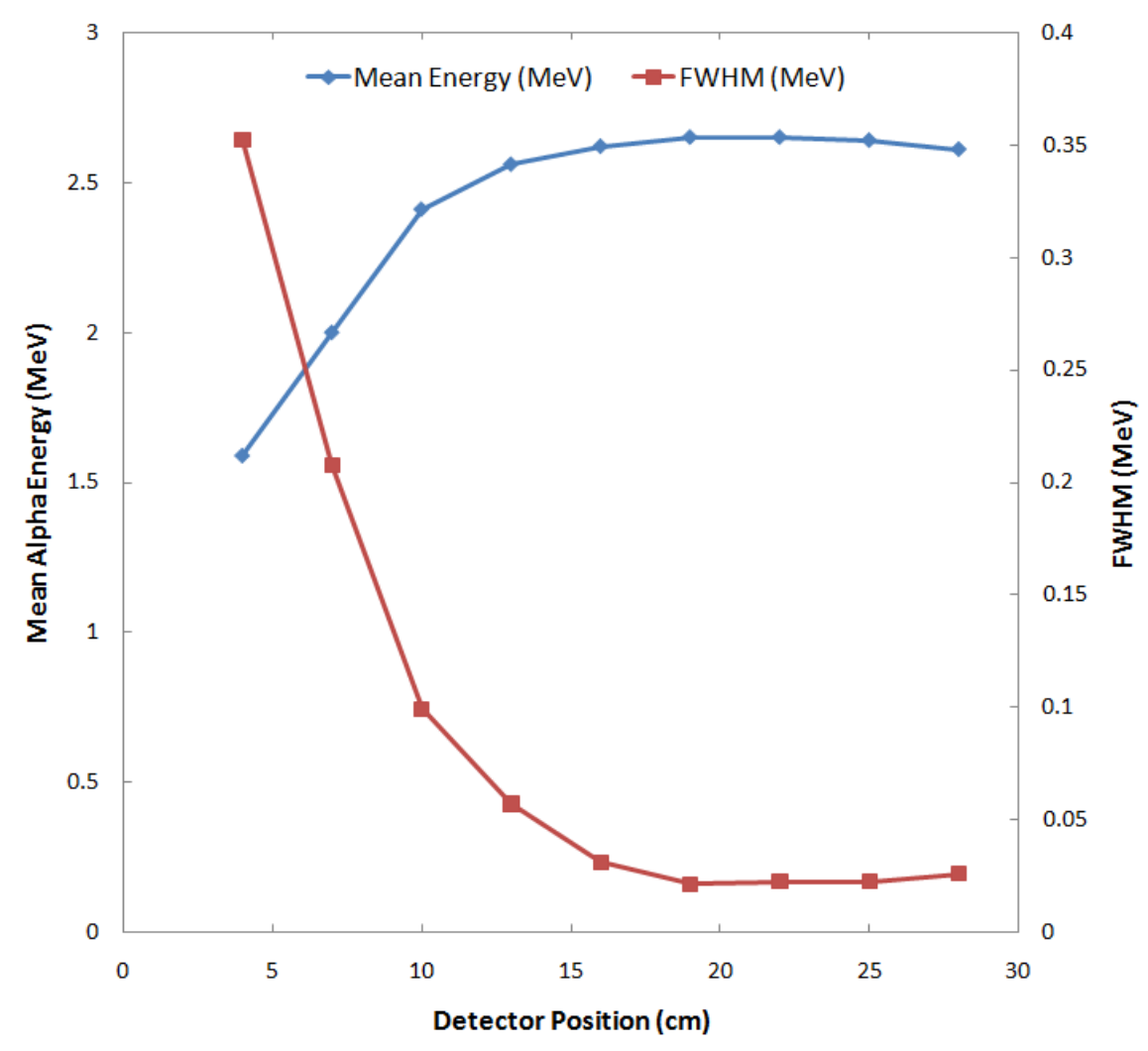

Figure 2-6. Calculated energy of the alpha particle as a function of the alpha-detector pixel position along the magnetic spectrometer axis. The energy resolution as a function of position is also shown.

A cutoff in the position of the detected alpha particles arises from the geometry of the Si detector array. The detectors are a cylindrical shell with an inner radius of $0.5 \mathrm{~cm}$ and an outer radius of $0.6 \mathrm{~cm}$. Thus, alpha particles emitted at shallow angles (e.g., $<15^{\circ}$ relative to the cylinder axis) will not be detected; they will follow a helical path down the axis without ever striking the $\mathrm{Si}$ detectors. Also, since the outer detector surface is at a finite radial distance from the axis, events will hit the surface of the detector at times slightly earlier than those predicted by naïve kinematic calculations based upon the spectrometer cyclotron period. This effect is more 
pronounced at smaller emission angles. This will create a small position-dependent bias in reconstruction of the neutron emission angle and the tagging time fiducial.

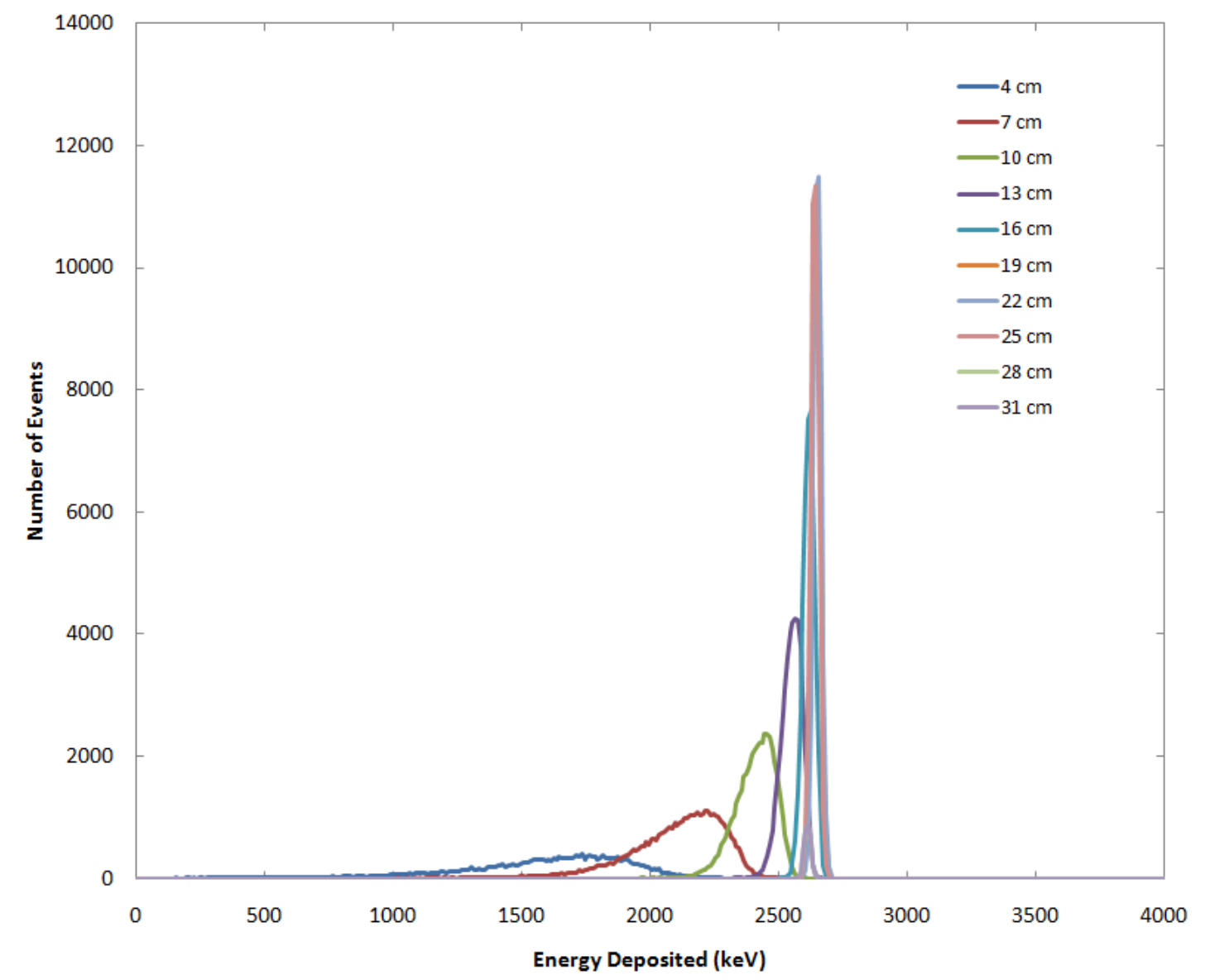

Figure 2-7. Alpha particle energy spectra from each Si detector, as a function of axial position from the target. The increase in dispersion of deposited energies at smaller positions (i.e. larger emission angles) is due to the distribution of alpha-particle energy losses in the target over the angular acceptance of the corresponding detector pixel. The pixel size for the spectra shown is $\mathbf{3 ~} \mathbf{~ m m}$.

Using the alpha particle hit position, the alpha particle emission angle can be calculated by determining the longitudinal and transverse velocity components for the alpha particle as emitted from the target foil. Once this emission angle is determined, the neutron emission angle can be reconstructed. Thus it is possible to reconstruct the neutron direction without measuring the alpha particle energy at the reaction vertex ${ }^{2}$. We simulated two different pixel geometries, $3 \mathrm{~mm}$ and $1 \mathrm{~mm}$ pixels, to evaluate the effect of pixel size on angular reconstruction. Each simulation case comprised $10^{6}$ (alpha-particle, neutron) emission events. The results of the neutron emission-angle reconstructions are displayed as functions of the pixel position in Figure 2-8. The figure shows that, based upon the detector geometry, it is possible to tag neutron emission angles in the range of $17-81^{\circ}$, which is approximately 4.5 steradians of angular reconstruction. For the 1

${ }^{2}$ Although, as noted above, a measurement of the alpha-particle deposited energy may improve the effective emission-angle resolution at large angles, by exploiting the correlation between this emission angle and energy loss in the target. We have not pursued this possibility in this work. 
mm pixel case, additional reconstruction is possible due to the larger number of pixels possible within the same geometric range. In this case, it is possible to reconstruct neutron emissions in nearly 5 steradians.

There is some bias in the reconstructed emission angle due to the fact that the surface of the alpha particle detector is $6 \mathrm{~mm}$ off the magnetic spectrometer axis. This bias is introduced in the current reconstruction algorithm due to the assumption that the alpha-particle transit time from target to detector equals $\mathrm{T}_{\text {cyc }}$, independent of axial position, for all detected alpha particles. However, the nominal $\mathrm{T}_{\text {сус }}$ calculated from the spectrometer magnetic field is the time at which particles returns to the axis of the cylinder. In our case, the particle impinges upon the detector surface slightly earlier than the nominal cycle time. We estimated the bias near the extremes of the detector axial position, 4 and $28 \mathrm{~cm}$ (to bound the bias), to be approximately $0.8^{\circ}$ and $0.4^{\circ}$, respectively. Additional algorithm reconstruction improvement will be necessary in order to eliminate this bias properly.

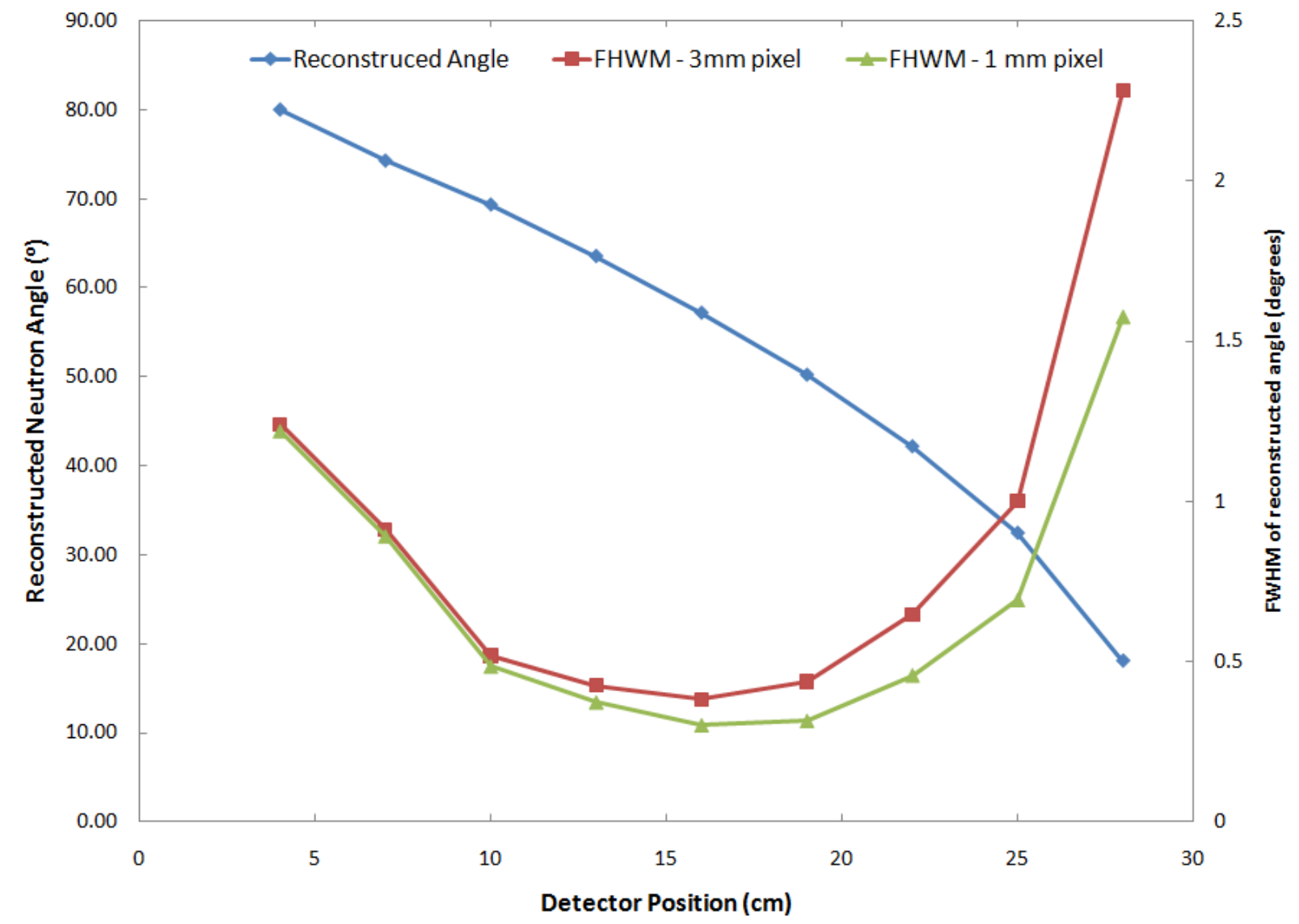

Figure 2-8. Simulation results for reconstructed neutron emission angle and associated angular resolution. Note that $2^{\circ}$ angular resolution or better can achieved over approximately $65^{\circ}$ of neutron polar angle.

As can be seen in Figure 2-8, the angular resolution of this reconstruction is quite narrow, with resolutions less than $1^{0}$ possible for a wide range of neutron emission angles. Significant degradation in the neutron angular resolution is evident at alpha particle axial positions smaller 
than about $5 \mathrm{~cm}$, where the combined effects of the pixel's angular acceptance and the finite beam spot size become important. Reducing the pixel size significantly improves the uncertainty in the neutron angular reconstruction. This is particularly true for neutron emission close to the spectrometer axis. Further improvement beyond the modeled cases is possible as well. If an order of magnitude decrease in the pixel size is assumed $(300 \mu \mathrm{m})$, the neutron angular uncertainty at $20^{\circ}$ is estimated to be improved to $0.9^{\circ}$ from $2.3^{\circ}$.

\subsection{Scenario Assessment: Application of nAPT}

As an example of the application of nAPT to container scanning applications, we modeled coincident detection of prompt gamma-ray emission from inelastic scattering of tagged neutrons in target samples inside a container. The interrogation technique modeled, pulsed fast neutron analysis (PFNA), is a neutron imaging technique that uses spectroscopy of the prompt gammaray as an indicator of the (isotopic) material content of the sampled voxel. The position of neutron scattering interactions leading to detection of a gamma-ray within a specified time window relative to the tagging fiducial is reconstructed from (1) the relative timing of the detected gamma-ray event in the gamma-ray detector with respect to the tagging time fiducial, and (2) the neutron emission angle as reconstructed from the information available from the neutron tagger. We refer to the gamma-ray detectors in the interrogation sensor system as the “X-arm” detectors as suggested by conventional usage in photon tagging facilities [Vogt 1993].

The objective of the simplified-geometry calculations reported here was to provide an initial estimate of the spatial reconstruction precision likely to be achievable with a tagged neutron generator featuring the angular emission and angular resolution properties modeled in sections 2.1 to 2.3 above. The possibility of making gross distinctions in target material atomic number and density is suggested in these initial calculations, which are not intended to fully exploit or represent the potential capability of an optimized PFNA interrogation approach for material discrimination and/or classification. We modeled the response of two arrays of NaI detectors, viewing two sides of a square container box subjected to inspection. We assumed only two detector arrays to represent a relatively portable sensor system, as opposed to three or four X-arm detector arrays, as would likely be instrumented in a facility-based, fixed-installation application. (There is no change in the energy resolution between the two cases, but the absolute detection efficiency for gamma-rays will decrease with the smaller number of detectors.) Figure 2-9 suggests the simulation geometry and typical results.

The position resolution of the interrogation method represents a tradeoff between two main measurement effects that exhibit opposite dependencies with respect to the distance of the inspected voxel from the neutron source. Finite resolution in the measured time of flight (TOF) and angular resolution of the tagged neutron both play roles in limiting the spatial resolution of the reconstructed interaction vertex. We assume that the TOF timing uncertainty includes contributions from the dispersion of tagged-neutron timing fiducial, the gamma-ray scintillation detector's output photosensor signal timing, and the intrinsic timing precision of the time-todigital converters (TDCs) used in the data acquisition. As a first approximation, these contributions will be constant over the TOF region relevant to the interrogation of the containerized samples. At larger total path lengths (neutron flight path plus prompt gamma-ray 
flight path), the TOF increases and the associated fractional error (equal to the ratio of the approximately constant TOF measurement error to the TOF) thus decreases. However, at larger distances of the sampled region from the neutron source, the importance of the finite taggedneutron angular resolution increases. Our simulations indicate that the neutron angular resolution dominates the spatial resolution for reconstructed positions on the far side of the container (relative to the neutron generator position). Finite timing resolution similarly dominates the position resolution at the near side of the container. On average, the spatial resolution for this simulation scenario is approximately $12 \mathrm{~cm}$ with reasonable timing resolution ( 2 ns assumed) and neutron angular resolutions (as detailed in section 2.3 above).

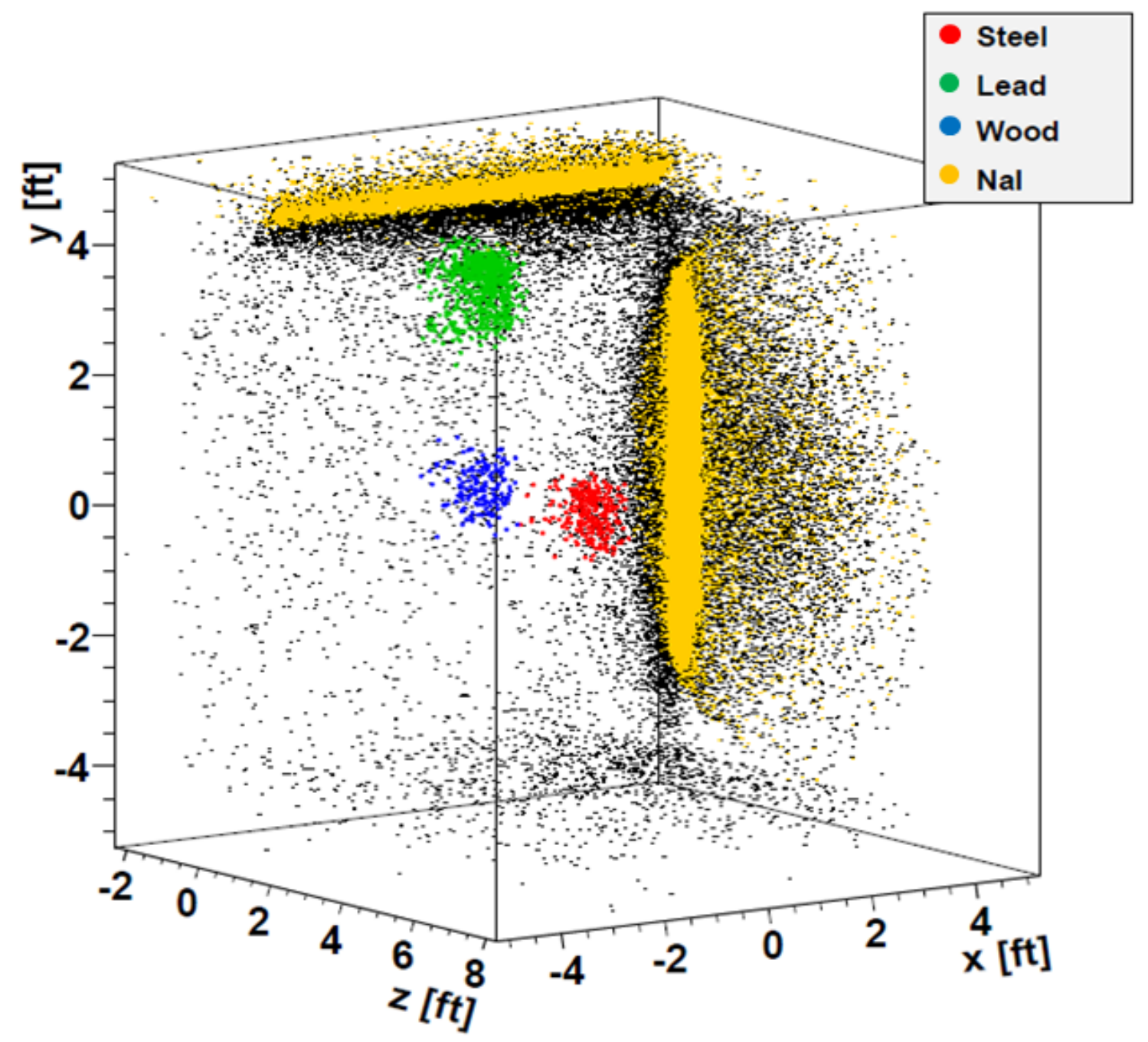

Figure 2-9. Simulated example application of tagged neutrons for cargo scanning. The three objects are color enhanced for improved visualization. Black dots correspond to scattering events from the cargo container wall.

We also assessed several count-rate aspects of the tagger electronics in this deployment scenario. Each run of the simulation code consists of $10^{8}$ total probe neutrons emitted into a $\sim 70^{\circ}$ cone. If we assume that the neutron generator produces $10^{7} \mathrm{n} / \mathrm{s}$ (emitted into $4 \pi$ steradians), then this 
tagged emission cone, approximately $33 \%$ of the full $4 \pi$ steradians, implies a neutron tagging rate of $3.3 \mathrm{MHz}$. (Note that this value is based on random tagging in time, and not a pulsed neutron generator.) Assuming this $3.3 \mathrm{MHz}$ tagging rate is distributed evenly over the 26-cm axial length alpha-detector pixel array assumed for the model of section 2.2, this amounts to a mean rate of roughly $30 \mathrm{kHz}$ per pixel at $3 \mathrm{~mm}$ pitch, and $10 \mathrm{kHz}$ per pixel at $1 \mathrm{~mm}$ pitch. A tagged rate of $3.3 \mathrm{MHz}$ also implies that the $10^{8}$ neutrons per simulation corresponds to about 30 seconds of collection time. Gamma rays above $1 \mathrm{MeV}$ generated by this tagged-neutron cone are detected in the simulated $\mathrm{NaI}$ bars at a rate of about $5.4 \mathrm{kHz}$ under these assumed neutron generator emission conditions.

We examined the impact of accidental coincidences between the gamma-ray detector and the neutron generator by modeling the time-of-flight distribution. We assumed the use of the $\mathrm{NaI}$ as the trigger (i.e., timing fiducial for the coincidence-time counting window) and feed the delayed neutron tagger timing into a multihit TDC. We assumed a time-of-flight window of $200 \mathrm{~ns}$. Thus, $200 \mathrm{~ns} \times 3.3 \mathrm{MHz}=0.66$, i.e., we expect about 2/3 of the time to have another neutron tag inside our (neutron, gamma-ray) time-of-flight window. These accidental coincidences will contribute a smoothly-distributed (roughly "flat") background between the true (neutron, gamma-ray) coincidence timing peak. Reducing the time-of-flight window to less than $100 \mathrm{~ns}$ should limit the accidental neutron hits/tags to an acceptable fraction.

\subsection{Neutron Generator Modeling: Conclusions and Future Work}

The use of a magnetic spectrometer design for neutron associated particle tagging has the potential to significantly increase the tagging rate and angular emittance for API neutron generators, potentially achieving approximately 4.5 steradians (versus $\sim 1$ steradian for current neutron tagging systems). Although not explicitly demonstrated in the model adopted for this preliminary design work, optimization of the detector geometry may further improve the taggedneutron emission solid angle to close to 5 or 6 steradians. These emission-angle increases should help facilitate development of advanced neutron tagging systems for relatively large-scale inspection applications, such as cargo screening. The use of currently available Si detector geometers with modest position resolutions will allow accurate reconstruction of the neutron emission angle, with resolutions less than $<2^{\circ}$ easily achievable, and less than $<1^{\circ}$ resolution possible for a wide range of emission angles. Many of the parameters used in this feasibility study are similar to those used in current neutron generators and magnetic solenoids, making this magnetic spectrometer concept feasible to construct with current technology. Finally, we performed basic simulations on the neutron magnetic spectrometer concept with NaI X-arm detectors in a cargo screening system and quantified potential position resolutions for materialspecific object identification within a cargo container using the design presented. Depending upon the depth of the target sample within the neutron generator's field-of-view, the largest influence in system position resolution is not the angular resolution of the neutron emission reconstruction, but the timing resolution of the $\mathrm{NaI}$ detectors and (neutron, gamma-ray) coincidence timing (assumed to be $\sim 2 \mathrm{~ns}$ ).

While we showed the potential of the magnetic spectrometer system in neutron associated particle tagging, additional studies will be required in order to better understand the potential of this device to serve in cargo screening and national security applications. The models only 
included the essential physical ingredients of the system, and additional detail (including more structural detail) will be required in order to better demonstrate the limits of the design. Current models only considered a homogenous axial magnetic field (i.e., solenoidal field) throughout the spectrometer interior. Additional studies are necessary to understand a variety of realistic magnetic field inhomogeneities and perturbations, in particular perturbations arising from structural materials immersed in the field. This is necessary in order to investigate the detailed alpha particle path within the solenoid. A detailed assessment of the impact of the magnetic field on the solid-state detector operation is also advisable, given anecdotal evidence from the magnetic spectroscopy community that differences in Si detector response and performance as a function of orientation of the detector surface with respect to an $O(1 \mathrm{~T})$ magnetic field may be anticipated. Finally, improved application modeling is necessary in order to better analyze the cargo screening applications and limitations as a function of magnetic spectrometer design. This would include incorporating more realistic cargo loadings and potential threat materials in a variety of shielding configurations. 


\section{Neutron Generator: Conceptual Design}

Associated particle imaging (API) using an electronic neutron generator (ENG) can serve as a very useful measurement technique for nuclear nonproliferation, nuclear security, and treaty verification. One implementation of the API technique uses gamma-ray spectrometry, measuring characteristic gamma rays produced through fast-neutron inelastic scattering to locate the location and spatial density of isotopes in an inspection region. Early work in the 1990s involving transportable API ENGs was spearheaded by researchers at Los Alamos National Laboratory and Argonne National Laboratory for nuclear and chemical treaty verification measurements [Hollas 1990, Morris 1990, Rhodes 1992, 1995]. Researchers at the Special Technologies Laboratory followed with important research improving the instrumentation, innovating many of the important developments needed for field-portable API instruments [Gordon 1990, Chichester 2005, Nebbia 2004]. Their early work focused mostly on performing inelastic-scatter prompt-gamma neutron activation analysis (PGNAA) using API-ENGs for detecting and locating high explosives, narcotics, and special nuclear material for hold-up ${ }^{3}$ assay applications [API 1998].

Others have followed in this area, mostly focusing on methods to perform stoichiometry in small-sized containers for the detection and localization of the COHN (carbon, oxygen, hydrogen, and nitrogen) elements found in explosives and narcotics [Blagus 2004]. For COHN analysis API instruments have also been developed for buried landmine detection, with general success under controlled conditions and a few cases showing success under real-world testing [Kuznetsov 2004, Lunardon 2004]. Interesting gamma-ray spectroscopy API applications outside of the security realm include whole-body elemental analysis and carbon analysis in soil for carbon sequestration studies [Mitra 1995, Ellis 2008, Wielopolski 2008]. API-related gamma-ray measurements have also been proposed that use API-ENGs in a time-of-flight configuration for high-resolution imaging, using a deuteron-deuteron (DD) neutron source rather than a deuteron-triton (DT) source, with high-resolving timing electronics that trigger events using inelastic-scatter gamma rays from materials such as aluminum (having reasonably lowerenergy, fast-neutron inelastic scattering reactions) [Evans 2002]. This approach allows onesided radiography for image reconstruction.

\subsection{Background on API Sensors}

Challenges exist with all current generation associated-particle neutron generators in relation to the alpha-particle detector. In some instances scintillators are placed inside the vacuum boundary of the sealed neutron tube in the ENG. These systems are often capable of delivering very high spatial resolution measurements for determining the transit vector of the alpha particles from the neutron-production target. However, these systems require the inclusion of a light transmitting window in the tube's vacuum boundary. These windows can be difficult to manufacture and often add considerable cost to the instruments. Further, the seals around these windows, separating the inner vacuum space and the outside world, represent a potential

\footnotetext{
3 "Hold-up" refers to the nuclear material deposited in the equipment, transfer lines, and ventilation systems of a processing facility.
} 
weakness in the systems overall ruggedness. In other implementations solid-state neutron particle detectors have been included inside the vacuum boundary of associated particle neutron tubes. Eliminating the use of large-area glass-to-metal seals these devices are inherently more ruggedized than the scintillator-based systems. However, the spatial resolution of these systems is considerably less acute than for the scintillator systems, and long term degradation in the detectors due to radiation damage may occur, which has the potential to degrade their performance over time. Further, with their inclusion inside the vacuum boundary, these detectors must be capable of withstanding the high-temperature associated with the neutron tube manufacturing process, which can introduce other challenges in their construction and manufacture.

The development of innovative alpha-sensor concepts has the potential of making API-ENG technology more suitable for the rugged trials of field-use.

\subsection{Conceptual Layout of the PNNL API ENG}

Reviewing the constraints of the modeling for the new-concept helical-path alpha-particle sensor API-ENG, a conceptual design layout (see Figure 3-1) has been developed for how these ideas might be incorporated into a practical sealed-tube electronic neutron generator. This concept envisions the use of a cold-cathode (Penning-type) ion source in a general API-ENG arrangement similar in some ways to previously developed instruments [Chichester 2005]. The external magnetic field would serve as the field inside the ion source, eliminating the need for a second magnet. The ion source would be maintained at positive high voltage, in the range of +90 to $+120 \mathrm{keV}$. Ions would exit the ion-source's exit cathode and then be accelerated across a small acceleration gap towards an outer sleeve, serving as a crude focusing electrode, with a small-diameter delimiting aperture on the beam axis. This aperture would help to reduce the beam diameter, limiting paths reaching the target, to ensure a small beam-spot at the target location. This is an inefficient approach for beam focusing but is simple to implement and avoids the complexity of using active electro-focusing components. More sophisticated ion source technology and beam focusing could be used in follow-on designs if needed.

The outer sleeve would also protect the solid-state alpha detector, located on its outer side, from the high electric-field region of the ion source. This is important to ensure a field-free region to avoid perturbations to the alpha particle trajectories due to varying user-selectable high-voltage changes. The generator would use standard hydride target materials and most-likely operate in a drive-in mode ${ }^{4}$ for initial deuterium-tritium loading. Electron suppression would be achieved through moderate target biasing, as illustrated in existing instruments [Chichester 2005]. The outer sleeve would be attached to an outer vacuum boundary casing made of a non-magnetic

\footnotetext{
${ }^{4}$ Drive-in mode refers to the manufacturing technique for the neutron tube using a bare/unloaded thin film of titanium. The gas reservoir is loaded with a 50\%-50\% mixture of both deuterium and tritium. Once the tube is fabricated and assembled into the system, it is then operated such that the D and T ions would be accelerated into the titanium thin film, where they would be implanted (similar to ion implanting for the semiconductor industry.) Over the course of 12-24 hours, sufficient D and T atoms have been implanted to reach equilibrium. Over the 12-24 hour period the generator slowly starts to produce more neutrons. Once equilibrium is reached, the generator then yields an essentially constant rate of neutrons and will do so for hundreds of hours to thousands of hours, depending upon the original thickness of the titanium film, which is slowly sputtered away during operation.
} 
steel such as 304SS. The vacuum boundary casing would be welded to a base flange which would then also attach to a high-voltage vacuum feed-through on the inside of the tube. This high-voltage feedthrough would be the main support for the inner ion-source column. Outside of the vacuum area, behind the feed through, would be a high-pressure area where a high-Voltage, Cockcroft-Walton type voltage multiplier would be located. To avoid high-voltage electrical discharges this section of the ENG would be filled with sulfur hexafluoride gas, to a nominal pressure of 80 to 120 psi. Standard electrical feedthroughs would be used to get control signals into the device. A gas-regulating control getter would need to be incorporated into the vacuum region of the tube; a logical place for locating this component would be near the main support flange, towards the down-stream end of the alpha sensor.

The base support flange would also include external mounting brackets to hold the high-strength electro magnet needed to constrain the alpha particles to helical trajectories inside the neutron tube. Figures 3-1 through 3-5 show various conceptual sketches of the ideas presented above.

The current project's notional design task has not addressed in detail the magnetic element requirements, which represent a substantial technical challenge even for a fixed-installation (as opposed to field-deployable) instrument. A large-bore, 5-Tesla solenoid would be expensive to buy and expensive to operate, either for electrical power and cooling of conventional magnet windings, or for cryogenics if superconductors are to be used. For example, assuming the resistivity of high purity copper at $20^{\circ} \mathrm{C}$ and an inside radius of $15 \mathrm{~cm}$, power consumption would be approximately $0.75 \mathrm{MW}$, and conventional water-cooling of the windings would be problematic. Space considerations for the windings themselves are also important. With a $15 \mathrm{~cm}$ inside radius and a $30 \mathrm{~cm}$ length, about 120,000 turns and 10 A would be required to produce a $5 \mathrm{~T}$ field. To fit in this volume, the wire cross sectional area would need to be $0.25 \mathrm{~mm}^{2}$, for a total resistance of $7.5 \mathrm{kohms}$. This simple estimate does not include the increase in length per turn as the coil diameter increases. In addition to power consumption and magnet size, magnetic field uniformity is another significant design issue. The magnet field fringes at both ends of a finite-length solenoid over a distance that is comparable to the solenoid diameter. Thus the magnetic field of a solenoidal winding configuration in which windings do not extend significantly beyond the spectrometer itself may not be sufficiently uniform to maintain a constant axial field (and thus, constant cyclotron period) over the length of the alpha spectrometer. On the other hand, extending the solenoid windings in the axial direction enough to give a uniform magnetic field may place a substantial amount of copper in the paths of the neutrons that are at large emission angles with respect to the axis, potentially reducing significantly the solid angle of the tagged neutrons. We note these implementation challenges here as issues of concern for a full engineering design and associated cost/benefit analysis. 


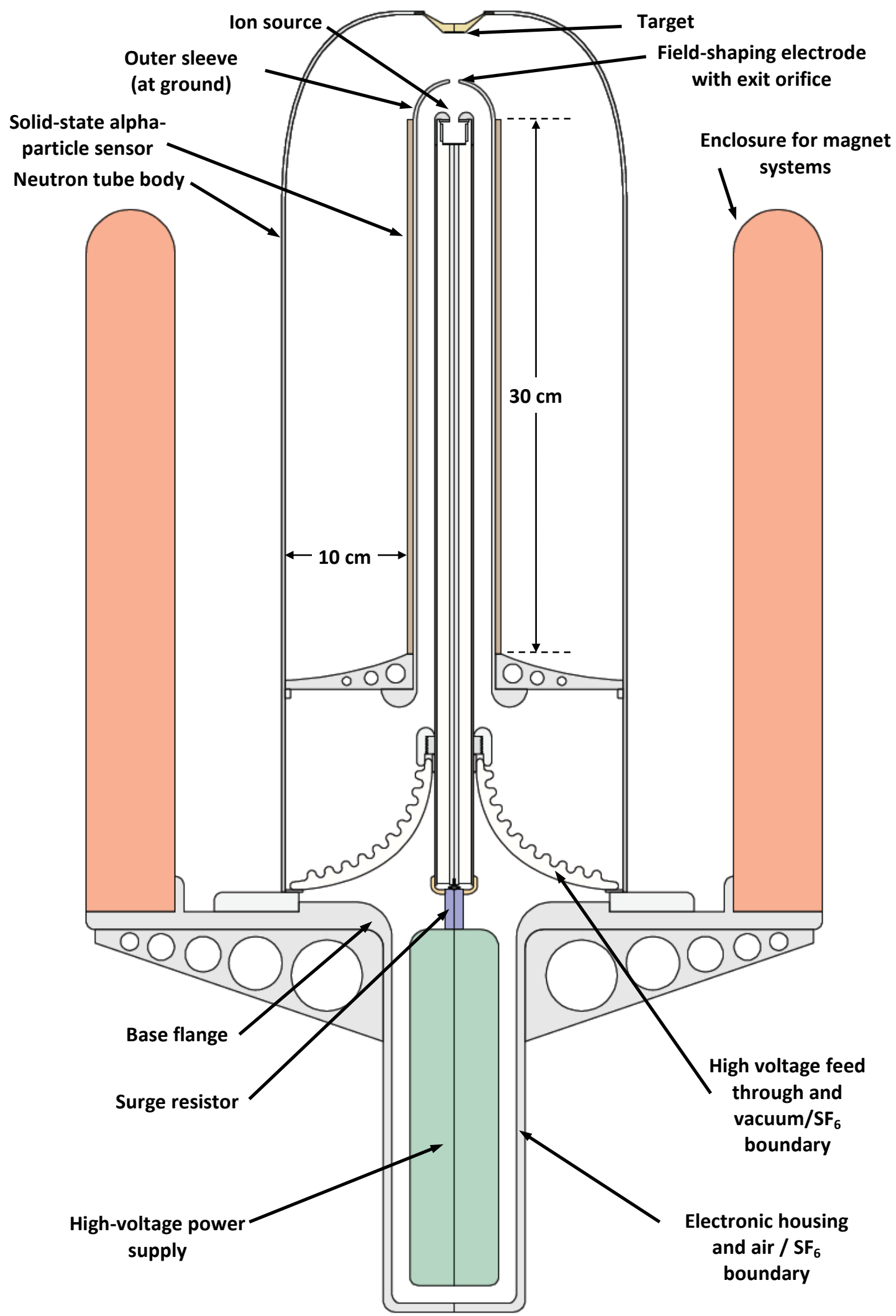

Figure 3-1 Conceptual cut-away drawing of a proposed layout for the PNNL API ENG. 


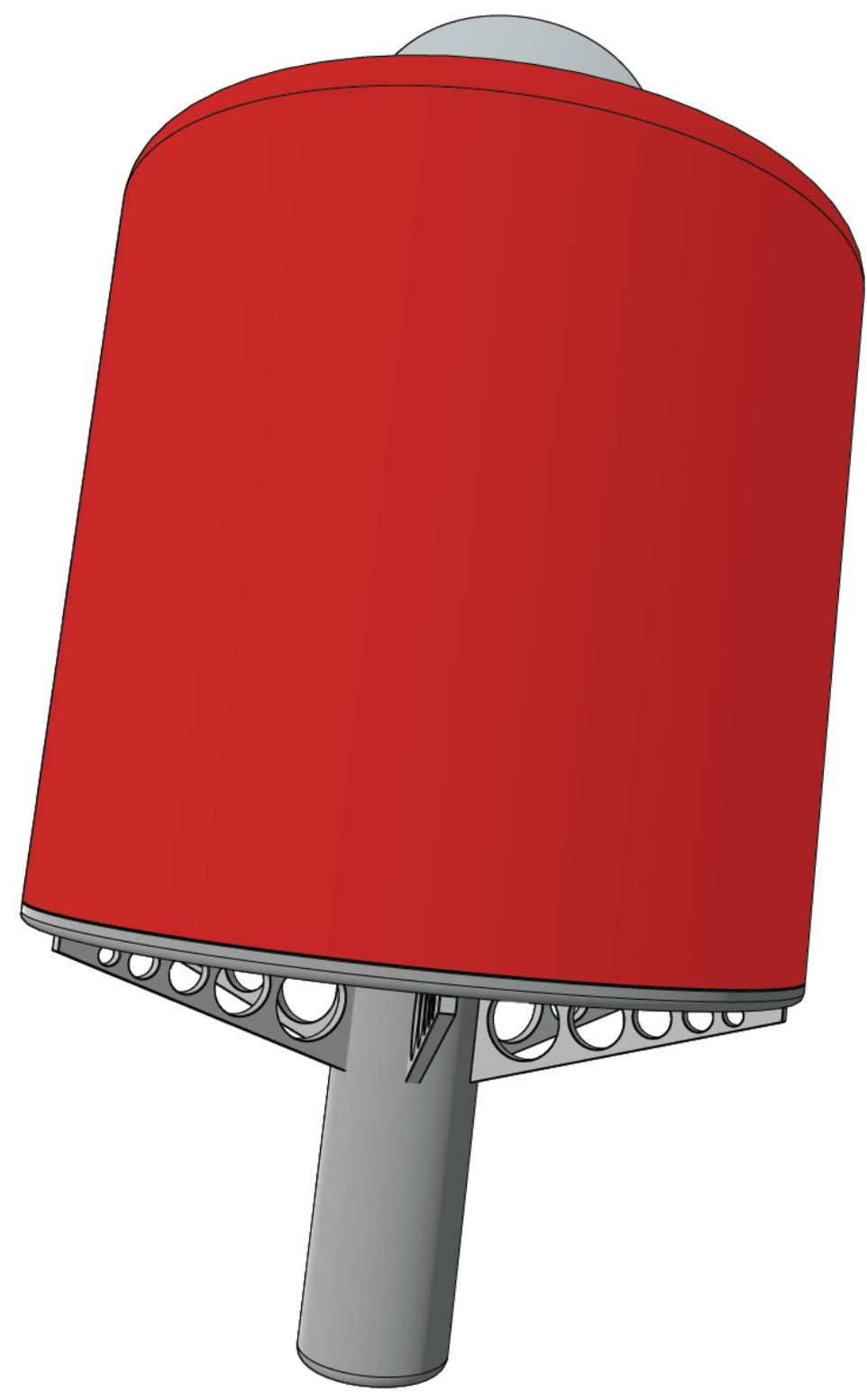

Figure 3-2 External view of a conceptual layout for an API ENG using a helical alpha trajectory sensor. 


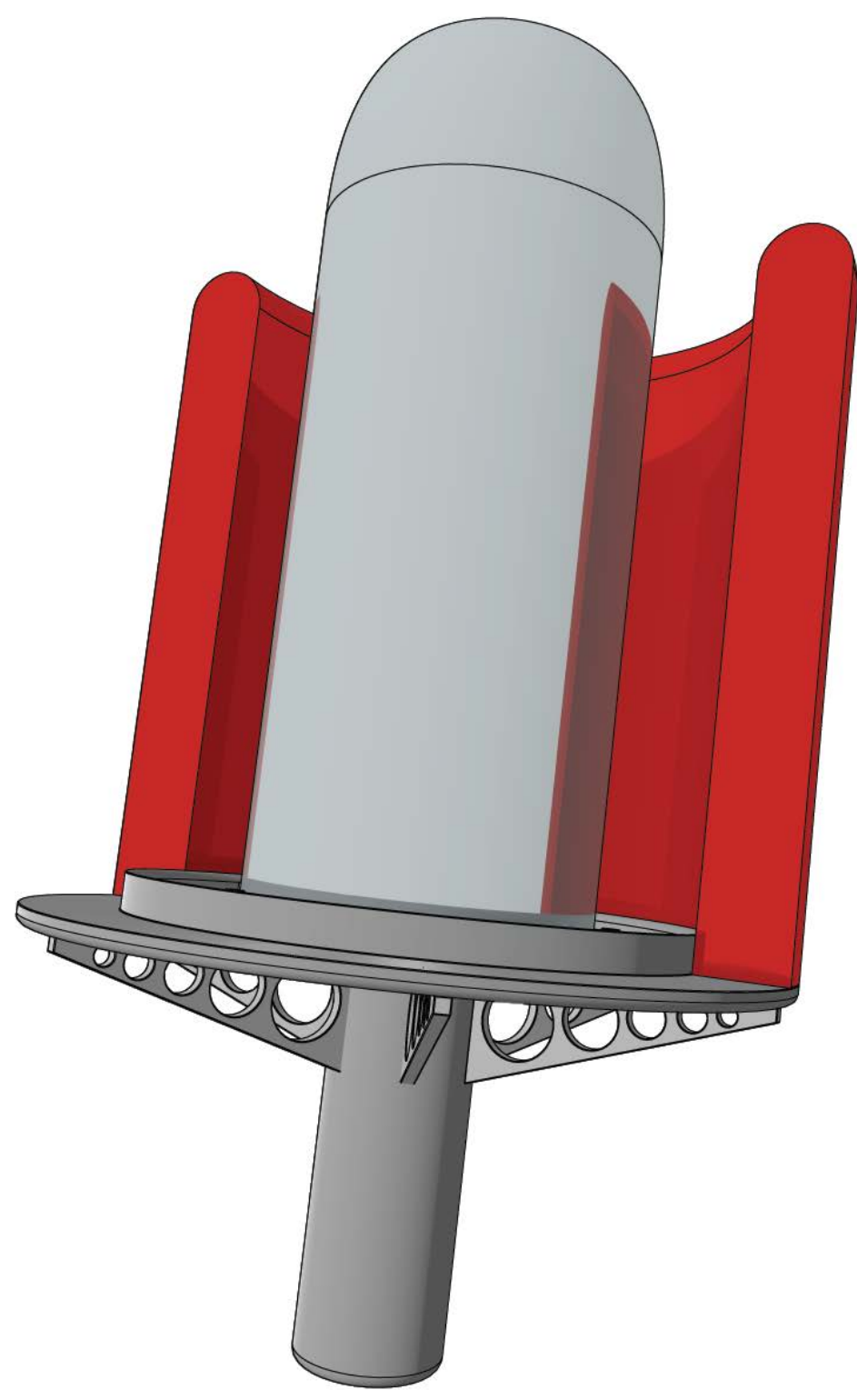

Figure 3-3 Cutaway view of a conceptual layout for an API ENG using a helical alpha trajectory sensor, showing the neutron tube. 


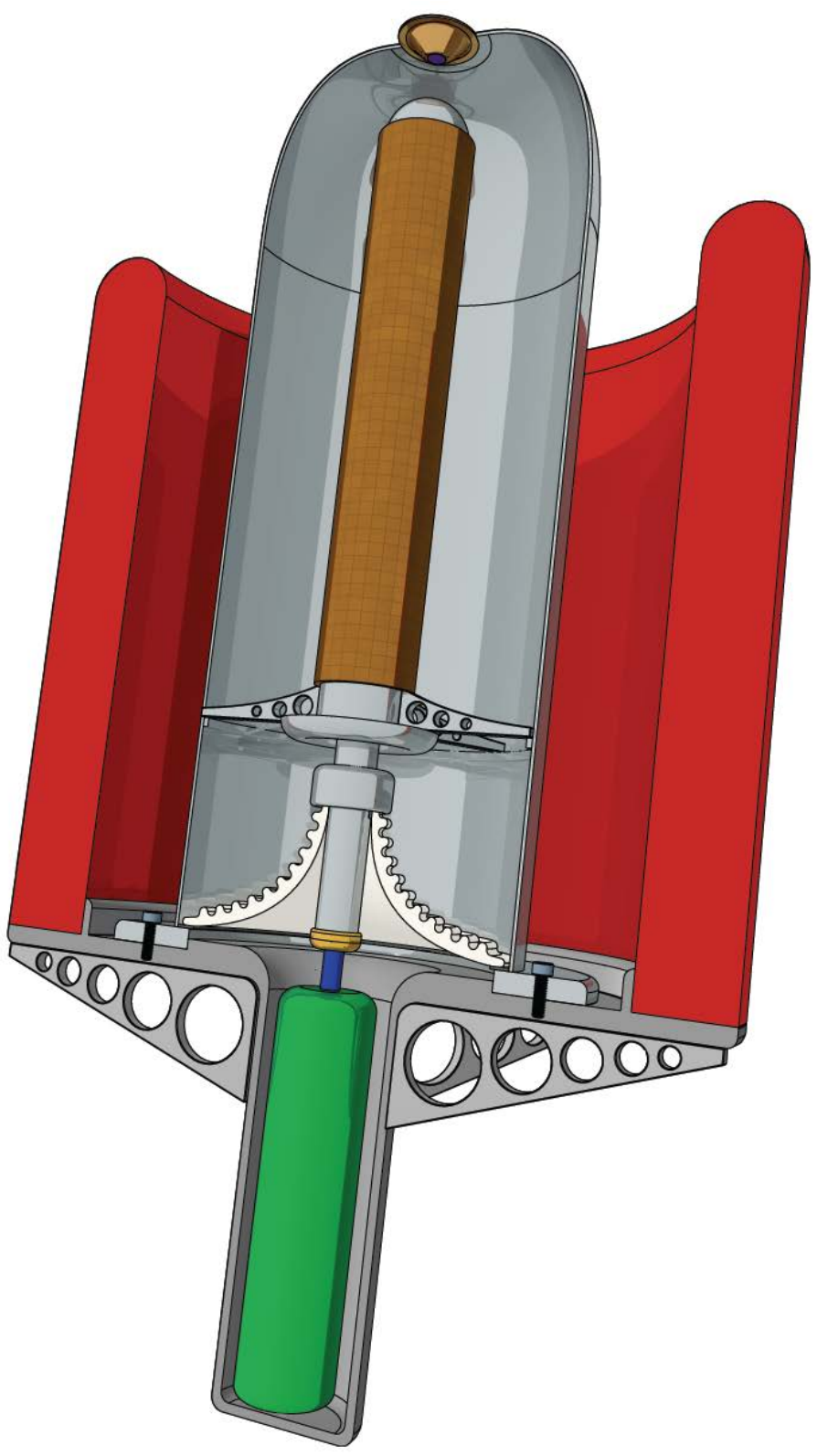

Figure 3-4 Cutaway view of a conceptual layout for an API ENG using a helical alpha trajectory sensor, viewing inside the neutron tube to see alpha-particle sensor. 


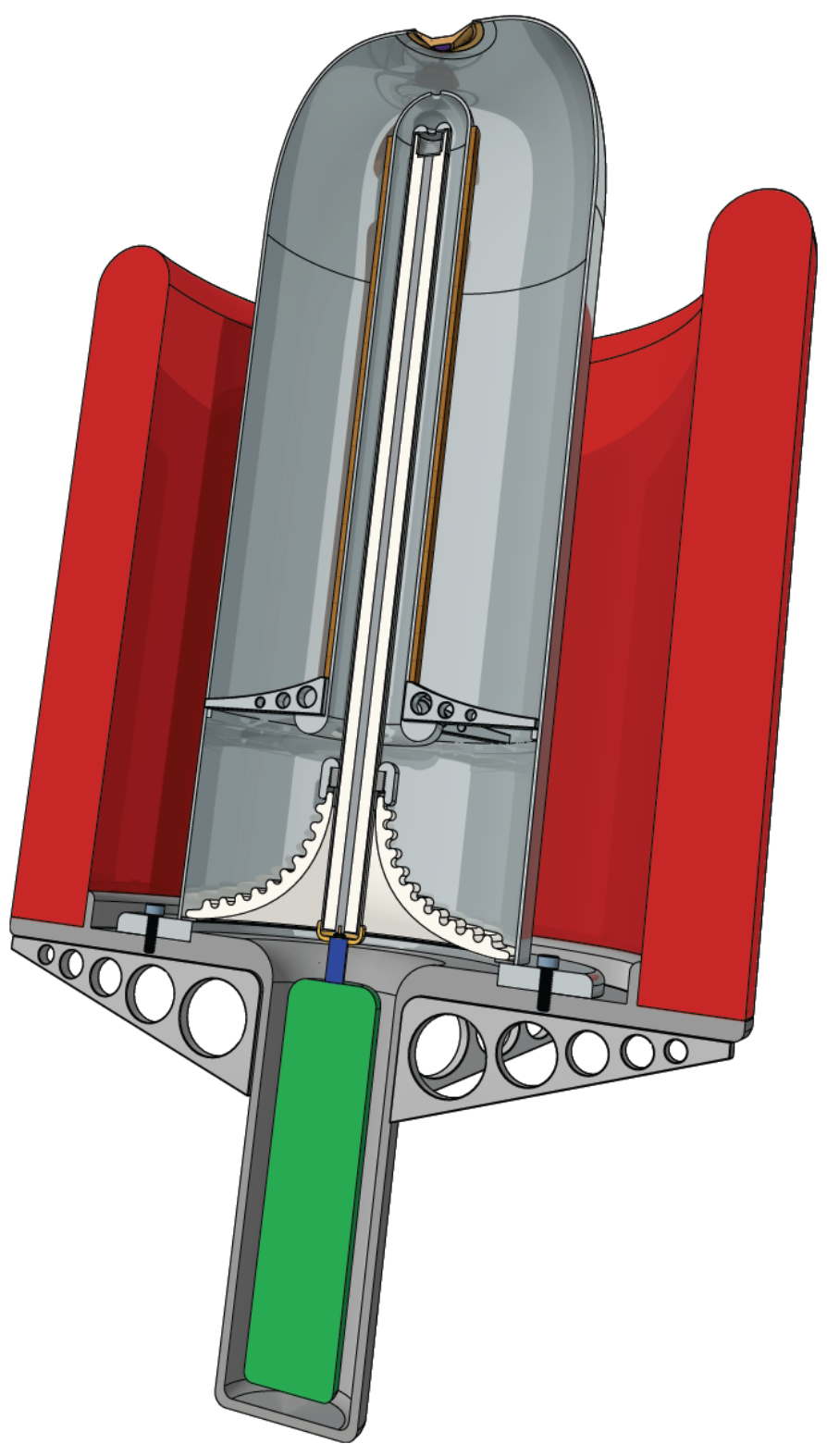

Figure 3-5 Cutaway view of internal components in a conceptual layout for an API ENG using a helical alpha trajectory sensor. 


\section{Photon Tagging: Modeling}

Previous sections of this report address the design and application of a tagged-neutron generator as a next-generation refinement and augmentation of the associated particle imaging technique. The remainder of this report addresses the application of tagged photon methods to active interrogation. In the neutron generator case, the primary purpose of the tagging technique (i.e. coincident detection of a particle associated with the production of the probe beam particle) is to extract information on the direction and timing of the emitted neutron. The "value-added" of this kinematic information is clear for imaging applications and background mitigation strategies, in which isolating the physical location of the interrogated sample by "electronic collimation" of the neutron beam offers potentially useful advantages. Demonstrating and quantifying "value added" of the application of an energy-tagged photon beam to active interrogation, however, represents a qualitatively different challenge. In this case, the utility of the event-by-event energy information available arises (potentially) from the ability to distinguish the materialdependent responses, as functions of interrogating energy, to the tagged photon probe. The aim is to optimize the selectivity of the interrogation method in distinguishing threat material (e.g. fissile materials) from benign materials, and to do so in a way that most efficiently uses the information content of the measured, energy-dependent response. This efficiency of information content analysis is essential because the tagged photon flux available in even the highest-rate, conceivable photon tagging designs will be several orders of magnitude lower than that available from conventional, broad-beam bremsstrahlung sources.

Given these considerations, the modeling task in this research project explores a potentially promising active interrogation approach, energy-tagged photoneutron emission, in which the known energy (within a specified, and potentially energy-dependent, resolution) of the tagged photon provides a means of mapping the energy-dependent neutron yield of the interrogated target. The energy of the (fast) photoneutron may also be measured in this technique, or the integrated yield over a specified neutron-response threshold may be recorded. In either case, however, the key observable of potential utility for discriminating the material response is the energy dependence of the photoneutron emission rate.

The concept explored in this modeling study exploits the fact that the photoneutron cross section varies significantly as a function of incident photon energy, especially in the 6 to $20 \mathrm{MeV}$ region. Figure 4-1 shows the photoneutron yield for six different materials chosen to represent a wide mass range and a reasonably representative sampling of materials of interest. Certainly within this limited set, the variation of yield with energy is qualitatively sufficient to identify each material. A tagged bremsstrahlung beam is capable of generating all of the different gamma-ray energies "simultaneously," i.e. within the same exposure of the sample to the interrogating beam. Photon tagging requires a detector which can measure the energy of the post-bremsstrahlung electron, thus giving the energy of the photon that was generated. A photon tagger uses a magnetic spectrometer to achieve this requirement by momentum analyzing the scattered electrons. 


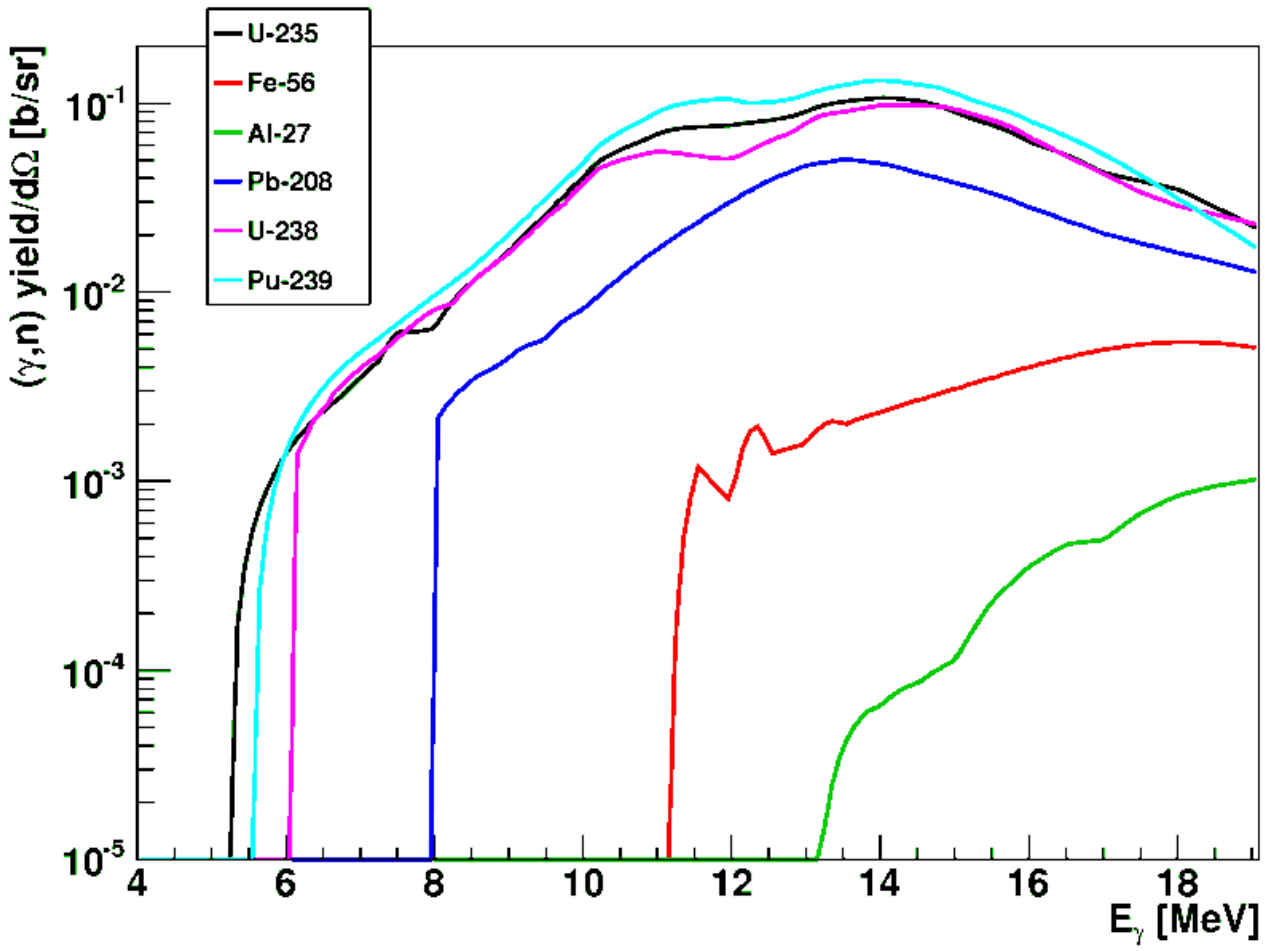

Figure 4-1: Photoneutron yield versus incident gamma-ray energy for six materials which represent a wide mass range.

For this study, we assumed the photon tagger to have the following properties:

- Electron beam

o Energy: $18 \mathrm{MeV}$

o Current: $20 \mathrm{nA}$

- $150 \mu \mathrm{m}$ aluminum radiator

- Flux from 6 to $18 \mathrm{MeV}$ of about $1.3 \times 10^{8} \mathrm{\gamma} / \mathrm{s}$

- Tagger channel energy width: varied from 100 to $500 \mathrm{keV}$

On the electronics side, the only assumption critical to the design is the use of multihit time-todigital converters (TDCs) which are started by the neutron arm and stopped by the (delayed) individual tagger channels. ${ }^{5}$ The multihit property is important since the rates will be rather high and the effect of accidentals becomes large, especially those of "stolen" true events when an accidental coincidence hit already in the delay pipeline stops the TDC before the true is able to stop it. In a single-hit TDC, that event is lost and must be corrected for. It is also possible that

\footnotetext{
${ }^{5}$ More explicitly, the time sequence of events is that a high energy electron scatters from the radiator, producing a photon. The photon travels to the interrogation target and produces a neutron via a photoneutron or photofission reaction. The neutron travels to the neutron detector, while the scattered high energy electron (following emission of the bremsstrahlung photon in the radiator) travels through a spectrometer and is momentum-analyzed. In practice, the neutron detector signal often provides the start to the TDC, while the tagger channels are delayed and fed to the stop inputs of the TDC.
} 
digital shape processing will be fast and cheap enough to use as well, in which case a TDC is not required but many channels of digitizer would be.

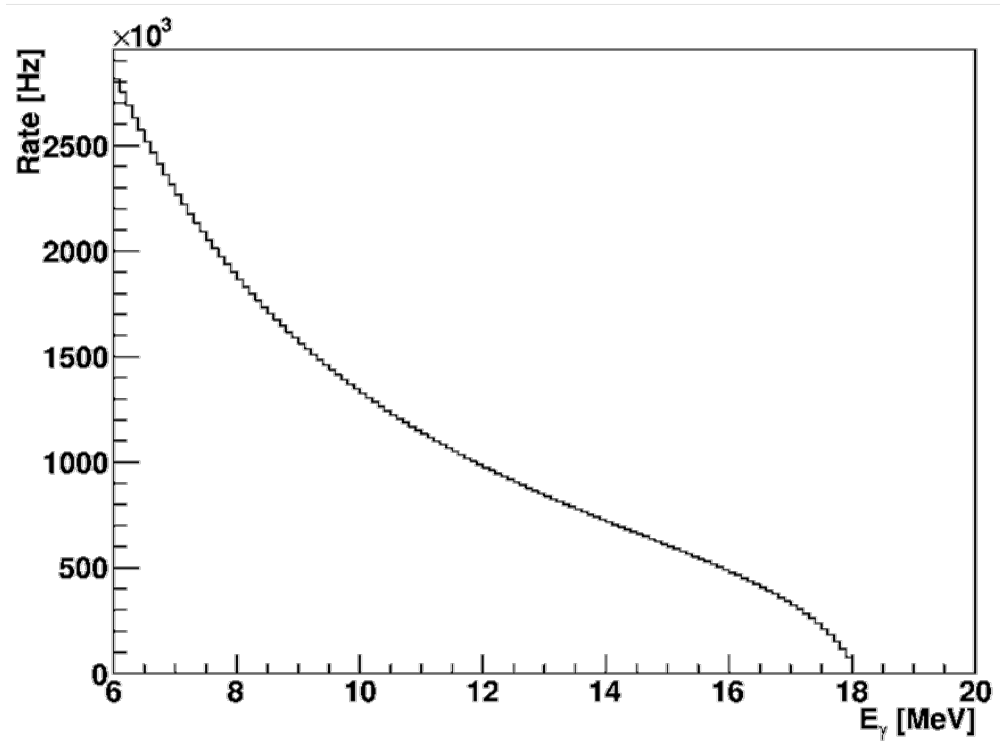

Figure 4-2: Tagged photon rate for $100 \mathrm{keV}$ wide tagger channels assuming the beam properties described in the text.

Figure 4-2 shows the tagged photon rate for $100-\mathrm{keV}$ wide tagger channels using the assumed properties of the electron beam and the radiator. With $100 \mathrm{keV}$ wide channels, the lower energy channels are already triggering at more than $2.5 \mathrm{MHz}$, leaving just $400 \mathrm{~ns}$ between pulses on average. Wider energy bins (corresponding to wider tagger focal plane detectors) will start to pose a rate problem at the lower energies as the rates increase. The exact upper limit will be a function of the electronics and beam conditions, but taggers are typically operated with individual channel rates of about $1 \mathrm{MHz}$. If a typical fast phototube has a signal width of $15 \mathrm{ns,}$ at $1 \mathrm{MHz}$ the dead time is $1.5 \%$. Past $10 \mathrm{MHz}$, the dead time is greater than $15 \%$ and becomes more difficult to correct accurately.

Figure 4-3 displays a schematic illustration of the scenario investigated in this report. Postbremsstrahlung electrons traverse the magnetic field and are detected by plastic scintillators (or scintillating fibers) arrayed along the focal plane of the magnet. The gamma-ray beam passes through the magnet to the active interrogation target. There is no collimation of the gamma-ray beam in the model scenario. Neutrons are then emitted from the inspected item via $(\gamma, n),(\gamma, 2 n)$, and $(\gamma, f)$ reactions and travel to a liquid scintillator detector. The neutron detector type was chosen based upon its ability to discriminate between gamma rays and neutrons on an event-byevent basis, while also providing a time-of-flight signal with minimal timing spread. 


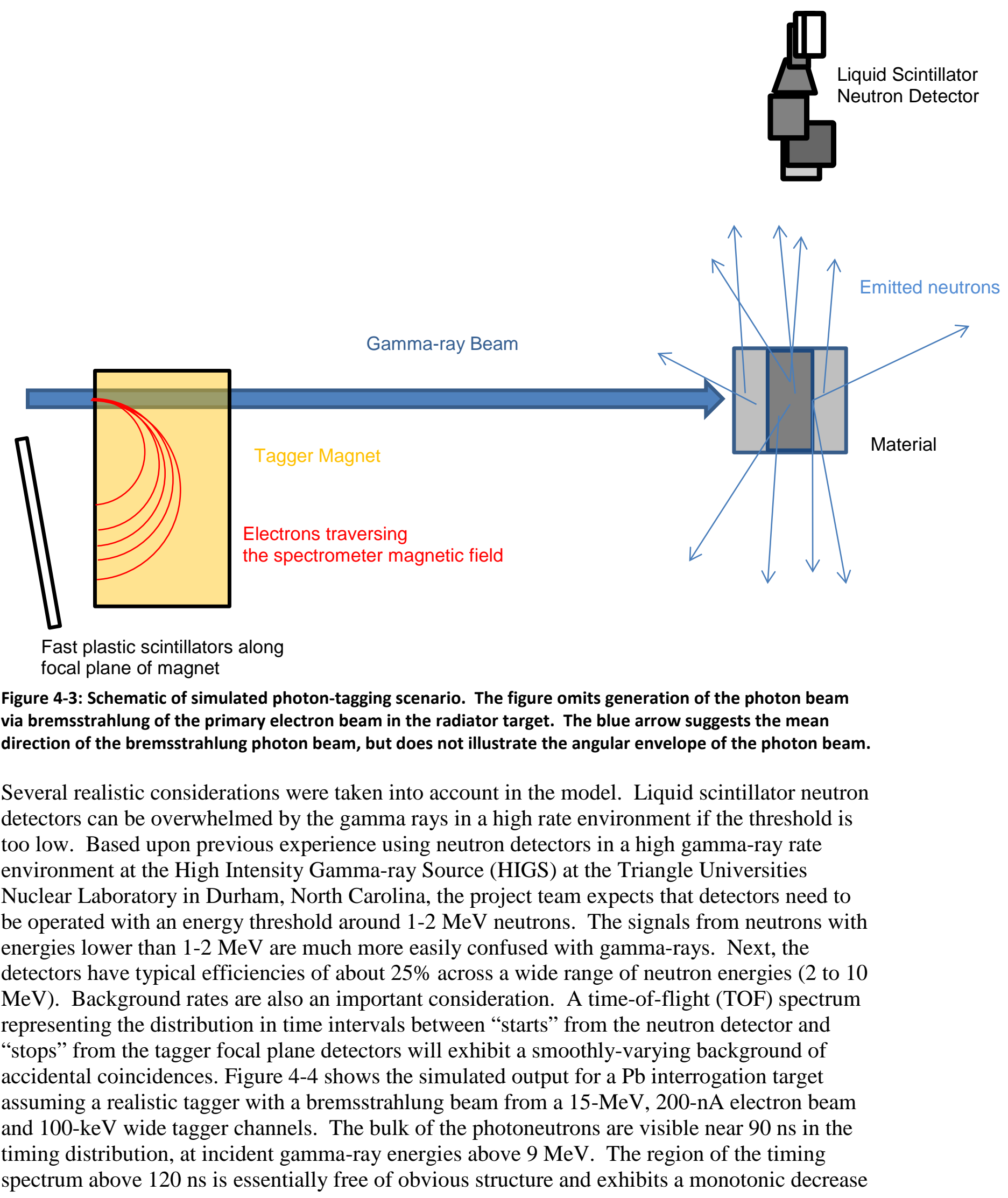


in counts with increasing gamma-ray energy. This is expected from the energy distribution of the incident bremsstrahlung beam (see Figure 4-2).

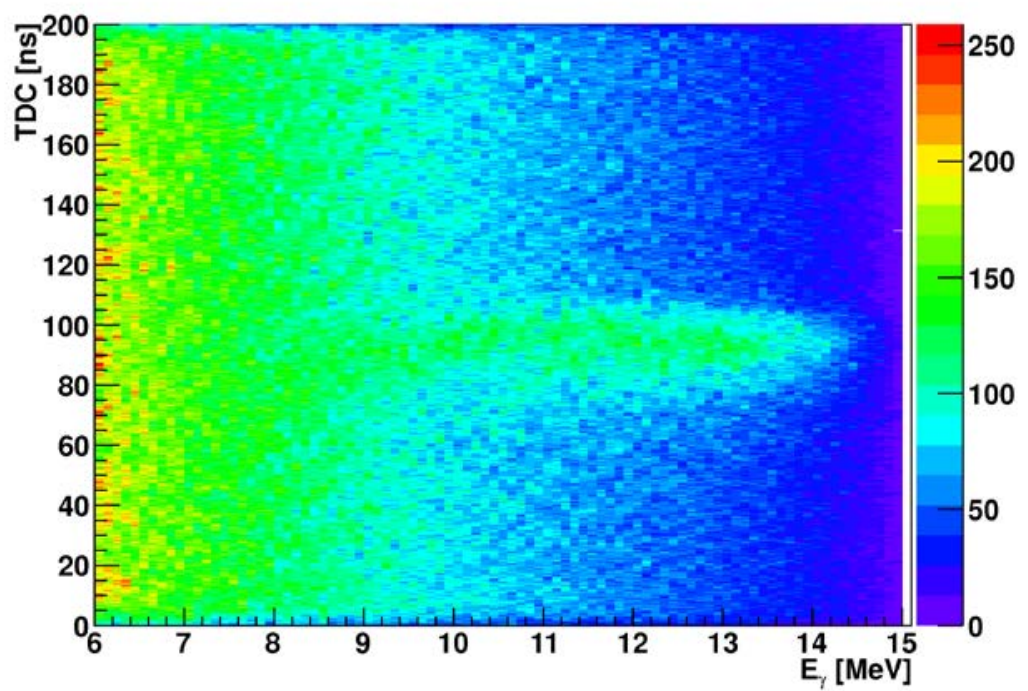

Figure 4-4: Simulated time-of-flight spectrum versus the tagged photon energy, $\mathrm{E}_{\gamma}$, for a $\mathrm{Pb}$ interrogation target. Photoneutrons are visible as a broad feature at about $90 \mathrm{~ns}$ in the timing spectrum and at incident photon energies above $9 \mathrm{MeV}$.

We simulated the time distribution of the detected gamma rays and neutrons in order to investigate the effect of the accidental background. Without accounting for this background, the apparent active interrogation signal registered in the photoneutron spectral region of interest will be too high (see e.g. Figure 4-5). While the background is significant, the out-of-time part (i.e. the portion of the distribution outside the neutron-gamma coincident window) can be used to subtract the background from the signature region. This then allows for a precise determination of the number of counts above background, which in turn allows for the precise determination of photoneutron yield (see Figure 4-6). 


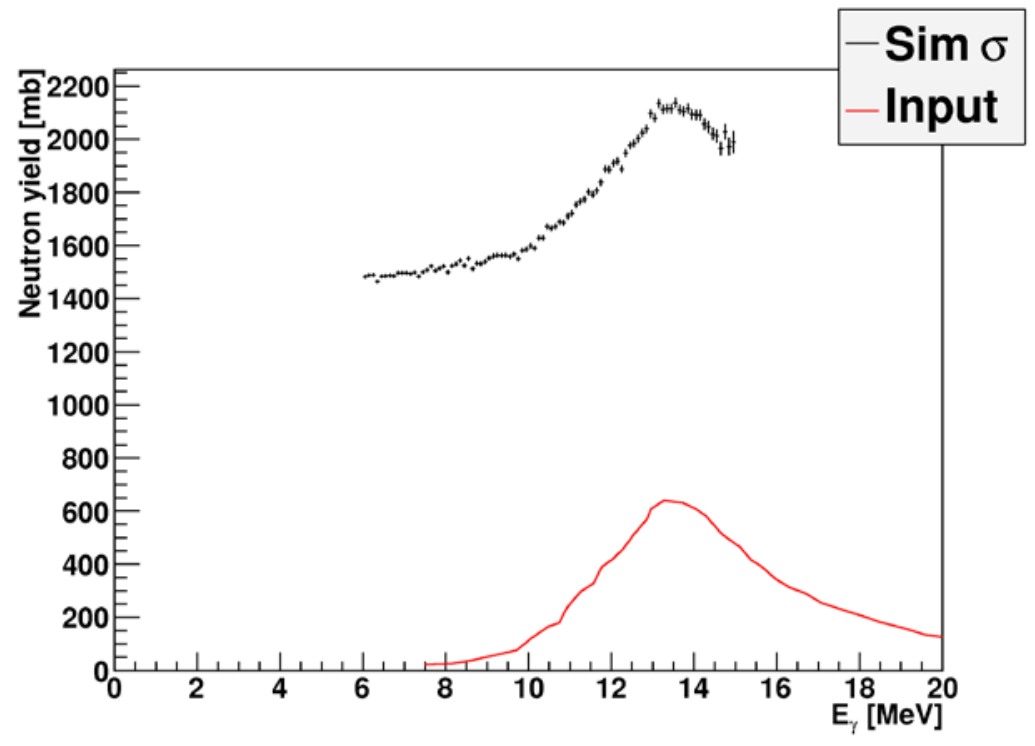

Figure 4-5: Simulation of the importance of accounting for accidental background in a photon tagging system. The black curve illustrates the apparent photoneutron yield (black curve) reconstructed from bombardment of a $\mathbf{P b}$ target without applying an appropriate background subtraction. The actual neutron yield distribution supplied as input to the simulation is plotted in red.

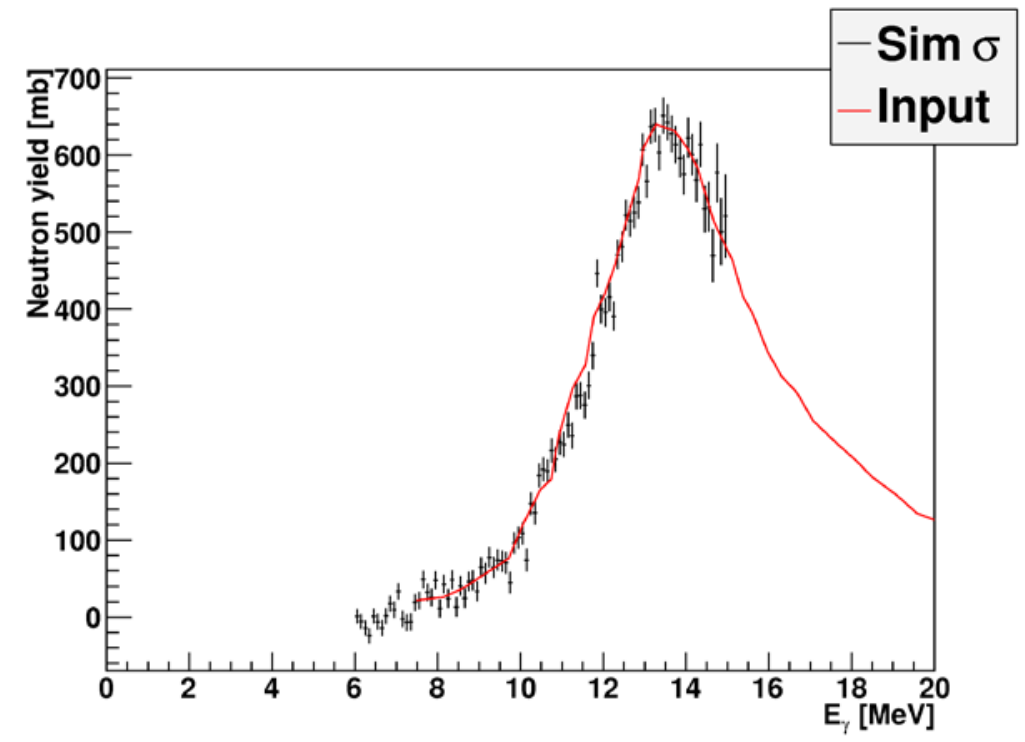

Figure 4-6: Reconstructed neutron yield on $\mathrm{Pb}$ after applying the out-of-time background subtraction. Note that in this simulation, the beam end point energy was $15 \mathrm{MeV}$.

As mentioned above, the photoneutron yield varies considerably with energy for different isotopes. In addition, the distribution of the outgoing neutrons varies as a function of neutron energy. The differences are clearly seen in Figure 4-7 where the two-fold photoneutron differential cross sections for ${ }^{56} \mathrm{Fe}$ and ${ }^{235} \mathrm{U}$ are compared. The cross section can be written as:

$$
\frac{d^{2} \sigma}{d E d \Omega}
$$


and is a function of incident photon energy as well as outgoing neutron energy. There is also a dependence on the polar angle which will be ignored in this analysis.

The reasons for the differences between the differential cross section shapes lies in the binding energy of the initial and residual nucleus as well as the dynamical properties of reaction. For instance, in ${ }^{56} \mathrm{Fe}(\gamma, \mathrm{n})^{55} \mathrm{Fe}$, for a given energy of the incident photon, there is a maximum energy that the outgoing neutron can have given the mass difference between ${ }^{56} \mathrm{Fe}$ and ${ }^{55} \mathrm{Fe}+\mathrm{n}$. That boundary forms the diagonal upper energy limit line. The peaks observed in the distribution are the result of different states in which the ${ }^{55}$ Fe residual nucleus can be left. In addition, $(\gamma, 2 \mathrm{n})$ processes lead to more complicated ways in which the energy can be shared between two outgoing neutrons. All of these processes occur in ${ }^{235} \mathrm{U}$, but in that nucleus, the fission process also occurs. The neutron energy spectrum from fission typically follows a Watt distribution which peaks around $700 \mathrm{keV}$ and rapidly falls off at higher energies. Importantly, there is no hard edge to the neutron yield distribution as in the $(\gamma, n)$ process.
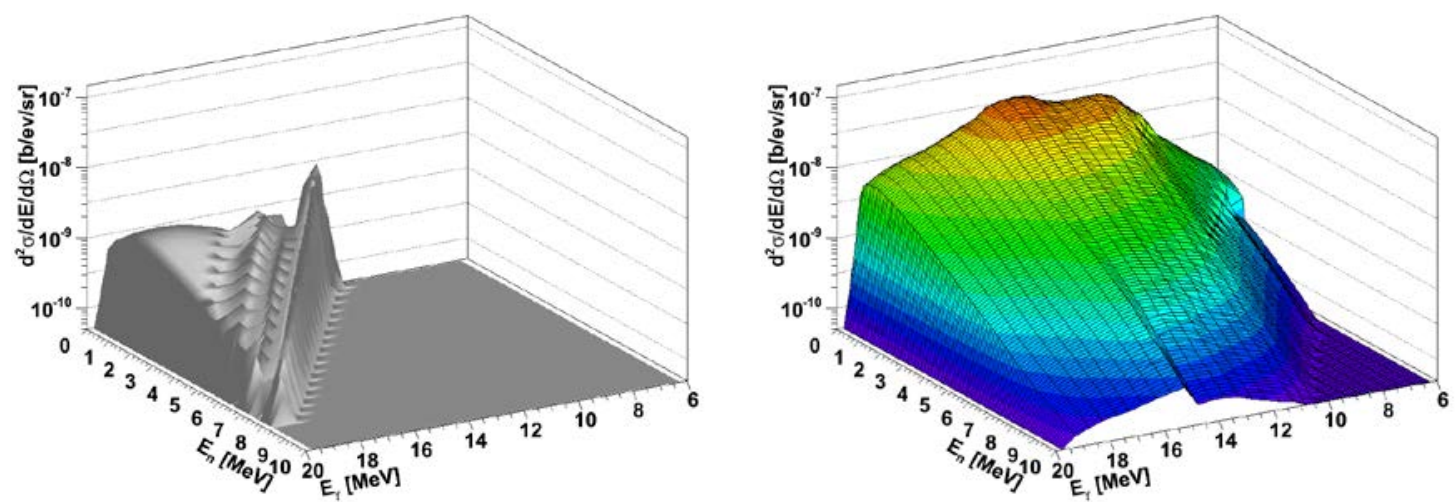

Figure 4-7: Comparison of the two-fold differential photoneutron cross section for Fe-56 (left) and U-235 (right). Data are from [Chadwick 2006].

The data plotted in Figure 4-7 are actually based upon model calculations since the data for these distributions are fairly limited. However, there are some unpublished neutron energy distributions from TUNL/HIGS for ${ }^{238} \mathrm{U}$ and $\mathrm{Pb}$ which show this same behavior.

The first pass at this study relied upon neutron spectra for ${ }^{238} \mathrm{U}$ and $\mathrm{Pb}$ generated with a polarized gamma-ray beam at the HIGS facility. However, additional experimental data on other targets were difficult to locate in the literature, thus limiting our initial study to only two materials. A concern was that such a limited database would allow a fitting routine to converge much more rapidly and precisely than a realistically sized one. We thus decided to expand the cross section data sources to include calculated cross sections. A study was commissioned by the IAEA [Oblozinsky 2000] to collect all of the photoneutron data available. The study identified 43 elements with 164 isotopes of interest, divided into categories as follows:

1. Structural, shielding, and bremsstrahlung target materials

2. Biological materials

3. Fissionable materials

4. Other materials. 
The authors of the study included recommended cross sections for each of the materials. In the intervening years, the National Nuclear Data Center (NNDC) database was updated and the lead author of the IAEA study has contributed to the ENDF database. While much of the data still need experimental verification, it is as good as the current models allow. As an initial test of a larger materials database (i.e. more than two items), ENDF data for ${ }^{27} \mathrm{Al},{ }^{56} \mathrm{Fe},{ }^{208} \mathrm{~Pb},{ }^{235} \mathrm{U},{ }^{238} \mathrm{U}$ and ${ }^{239} \mathrm{Pu}$ were retrieved from the NNDC database [Chadwick 2006]. Finding a common format was a challenge but eventually, it was determined that the ENDF MF=6, MT=5 format would give the information needed. That particular quantity is the double differential cross section,

$$
\frac{d^{2} \sigma}{d E d \Omega} \text {, }
$$

and is given in units of barns/eV/steradian. The plotting capability of the ENDF database reports the differential cross section as a function of incident gamma-ray energy in the laboratory, emitted laboratory neutron energy and neutron angle. The gamma-ray energies varied depending on the target, but the emission angles were always $10^{\circ}, 60^{\circ}$ and $150^{\circ}$. While the tabulated differential cross sections exhibit some anisotropy, this is ignored in the rest of the present study. We assume the neutron detectors will be placed at $90^{\circ}$ degrees, and thus use only the cross sections tabulated at the closest angle, $60^{\circ}$.

We then generated a two dimensional set of differential cross section versus incident gamma-ray energy $\left(E_{\gamma}\right)$ and neutron energy $\left(E_{n}\right)$ for each isotope in the database, for the $60^{\circ}$ neutron data only. Interpolation on this grid is required in order to evaluate the cross section at arbitrary $\mathrm{E}_{\gamma}$ and $E_{n}$. However, standard Cartesian or Delaunay interpolation is insufficient for this type of data. That interpolation does not account for the structure imposed by the underlying physics. For these reactions, as the $\mathrm{E}_{\gamma}$ increases, $\mathrm{E}_{\mathrm{n}}$ also increases. The ENDF-6 format manual [Oblozinsky 2009] suggests the use of an interpolation routine called unit-base transform (UBT) interpolation. This interpolation routine takes the range of neutron energies into account at each gamma-ray energy and generates a much smoother (and more physical) distribution. Figure 4-8 shows a comparison of the results using the two interpolation methods on the ENDF data for ${ }^{208} \mathrm{~Pb}$. The plot on the right is much smoother and exhibits more fidelity to the expected physical variations, especially for the finer structures at higher neutron energies.
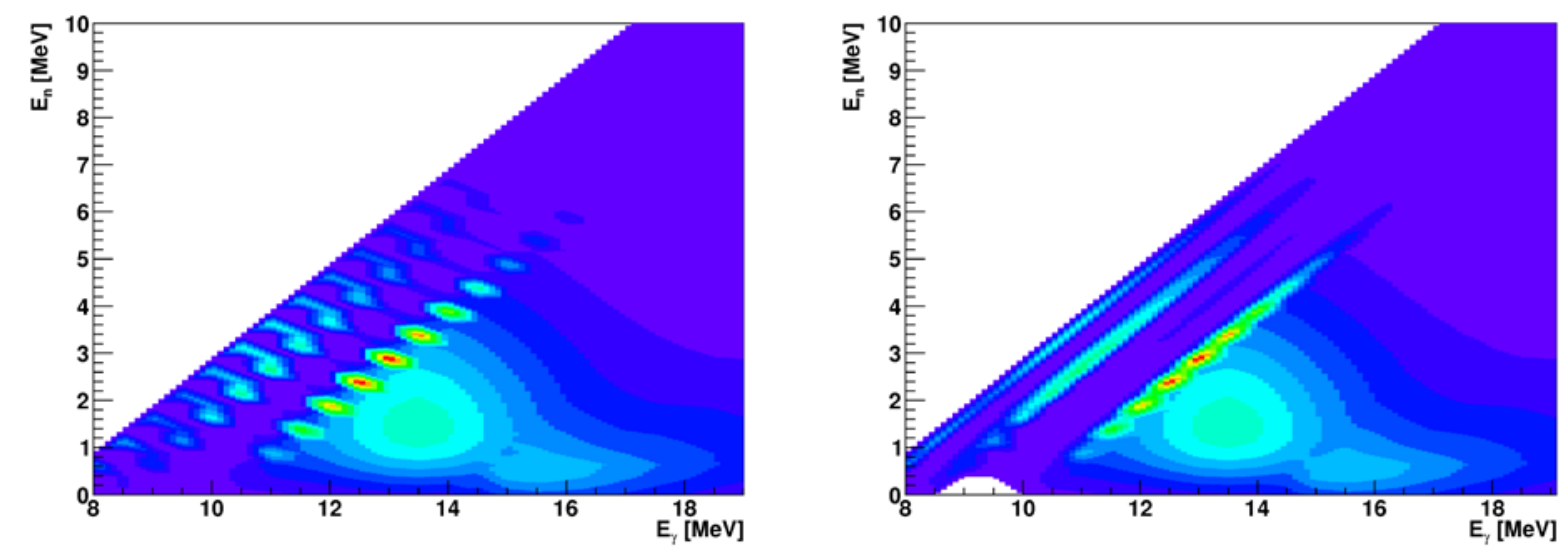

Figure 4-8: $E_{n}$ versus $E_{\gamma}$ photoneutron yield distributions for ${ }^{208} \mathrm{~Pb}$. On the left the tabulated cross section data are interpolated using the Delaunay method. On the right, the unit-base transform method is used. The yields 
are plotted in arbitrary units; the purpose of this comparison is to illustrate the qualitative difference in structure of the yields for the two interpolation methods.

To test the effect of using only the $60^{\circ}$ data, we calculated the total cross section from the ENDF differential cross section assuming isotropy and then compared with the neutron yield as given in Dietrich and Berman [Dietrich 1988]. The results are plotted in Figure 4-9. As is clear in the plots, the agreement is fairly good ( $20 \%$ maximum discrepancies) over the entire set of isotopes and quite good (a few percent) for some isotopes. There are also no obvious trends in this relatively limited data set indicating systematic deviations of the ENDF data, either high or low, relative to the Dietrich and Berman yields. It also appears that the neutron multiplicity is taken into account in the tabulated data, implying that no additional multiplicity information is required as input to our simulations. These data are appropriate for our simulations of a realistic tagger, since detected neutron events will in principle include emission from photo-reactions generating neutrons with any physically possible multiplicity. Thus generating simulated photoneutron emission according to these multiplicity-summed distributions should not introduce an appreciable bias in our modeled tagged-event rates, computed as a function of tagged photon and emitted neutron energies. 

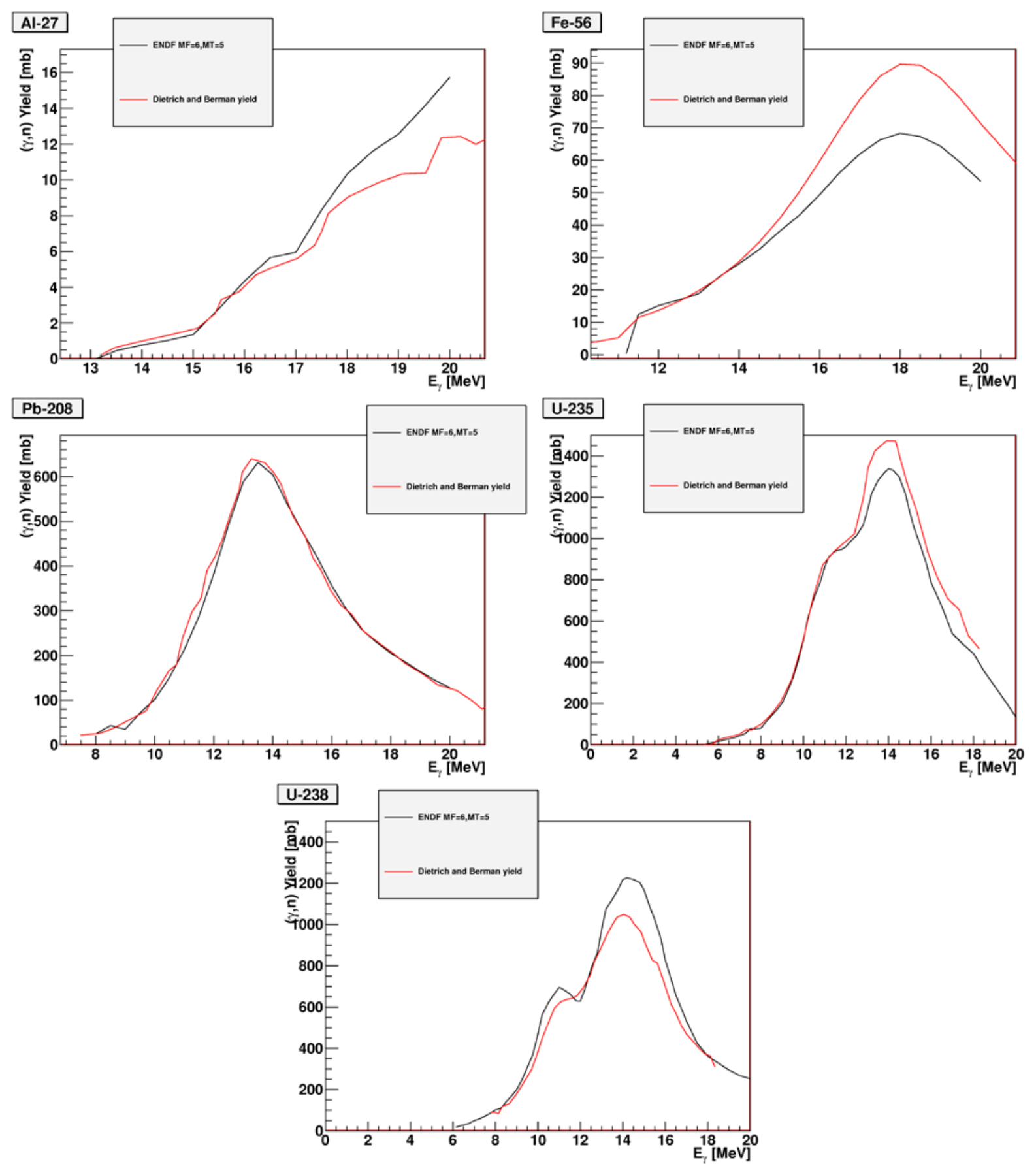

Figure 4-9: Comparison of ENDF evaluated photoneutron yield data with the yield from [Dietrich 1988]. In order to make this comparison, the ENDF cross section tabulations have been integrated over neutron angle from the $60^{\circ}$ data assuming isotropy.

We model the potential materials-discrimination capability of the tagged photoneutron approach as follows. For the two dimensional simulation (i.e. neutron yield dependence upon both bombarding photon and outgoing neutron energies taken into account), we load the ENDF database from files, smoothly interpolate with the UBT method, and weight the neutron yield by the theoretical bremsstrahlung distribution. We then process the resulting photoneutron yield to 
take into account smearing of the apparent, measured neutron energy stemming from the finite timing resolution of the tagging system. The energy resolution for any given neutron energy bin can be calculated given the $50 \mathrm{~cm}$ distance to the detectors and an assumed timing resolution of 1 ns $(1-\sigma)$. We generate one of these interpolated and energy-smeared yield vs. $\left(E_{\gamma}, E_{n}\right)$ distributions for each isotope included in the simulation. We then use these yield distributions to calculate the number of neutrons with energies greater than $2 \mathrm{MeV}$ as detected by a fast neutron detector with $25 \%$ intrinsic efficiency and diameter $12.7 \mathrm{~cm}$ (5 inches) located at a standoff of 50 $\mathrm{cm}$ from the target. The mean detected counts are then repeatedly randomized assuming Poisson counting statistics in order to produce simulated instances of actual finite-time measurements. The simulated target can be composed of an arbitrary amount of each of the materials in the database. In order to interpret the synthetic neutron yield in terms of target composition, we pass the synthetic data to a fitting routine. The routine finds the combination of database materials which minimizes the difference between the calculated and observed counts in the synthetic spectrum. The Monte Carlo calculation repeats the synthetic spectrum generation and fitting procedure many times $(O(100)-O(1000)$ trials) using different random counts each time to build up a history of fitted target composition parameters. The mean and root-mean square (RMS) of all of these fitting parameter results are then plotted and compared against the input parameters. For this study, the six isotopes mentioned above were included in the database: ${ }^{27} \mathrm{Al},{ }^{56} \mathrm{Fe},{ }^{208} \mathrm{~Pb}$, ${ }^{235} \mathrm{U},{ }^{238} \mathrm{U}$, and ${ }^{239} \mathrm{Pu}$.

Figure 4-10 shows the fitting results for a scenario with a target of $6.0 \mathrm{~cm}$ of $\mathrm{Pb}$ and $7.2 \mathrm{~cm}$ of ${ }^{238} \mathrm{U}$. This corresponds to an approximately $7-\mathrm{kg}$ cube of ${ }^{238} \mathrm{U}$ with $3 \mathrm{~cm}$ of $\mathrm{Pb}$ on either side. No attenuation of the incident gamma-ray beam is assumed, nor is any secondary scattering of the outgoing neutrons taken into account. All of the error bars are the 1- $\sigma$ uncertainties arising from counting statistics and derived from the minimization routine. In both the 1D (i.e. energyintegrated neutron yield cross section as a function of photon energy taken into account) and 2D (i.e. neutron yield cross section as a function of both photon energy and neutron energy taken into account) fitting approaches, the material composition reconstruction algorithm determines the correct amounts of the two materials within four minutes of simulated measurement time. The 2D approach exhibits a larger systematic bias for smaller count times than the 1D approach, although the reconstruction parameter precisions for any given dwell time are smaller in the 2D approach. Both methods are able to determine that the amounts of the other database materials in the target are consistent with zero. The large uncertainties on the aluminum thickness arise from its much smaller cross section for photoneutron yield. The fitting routine is able to vary the amount of aluminum significantly without a significant effect on the quality of the fit due to the predominance of the yields from higher-Z materials. The source of the bias in reconstructed ${ }^{238} \mathrm{U}$ content at low exposure time in the 2D approach has not been thoroughly investigated. It does appear qualitatively that the two dimensional approach is better able to constrain the values of the target composition. In any case, with sufficient counting time the uncertainties can be diminished further. For instance, at 900 seconds dwell time and $500 \mathrm{keV} /$ channel photontagging resolution, the $2 \mathrm{D}$ approach determines the aluminum thickness of the simulated target to be $0.18 \pm 0.33 \mathrm{~cm}$. 
Tagger $\Delta \mathrm{E}=500.0 \mathrm{keV} / \mathrm{chan}$

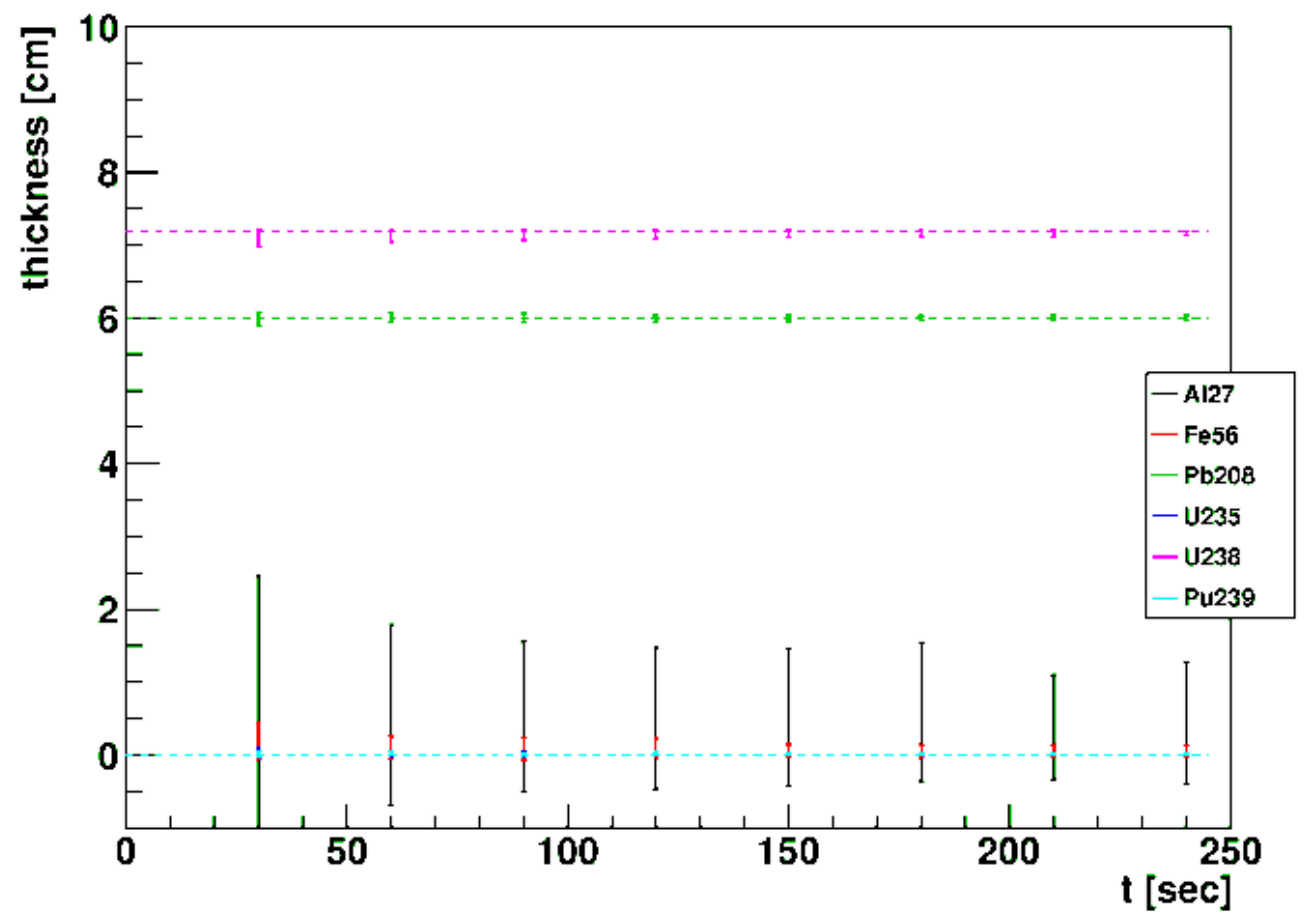

\section{Tagger $\Delta \mathrm{E}=500.0 \mathrm{keV} / \mathrm{chan}$}

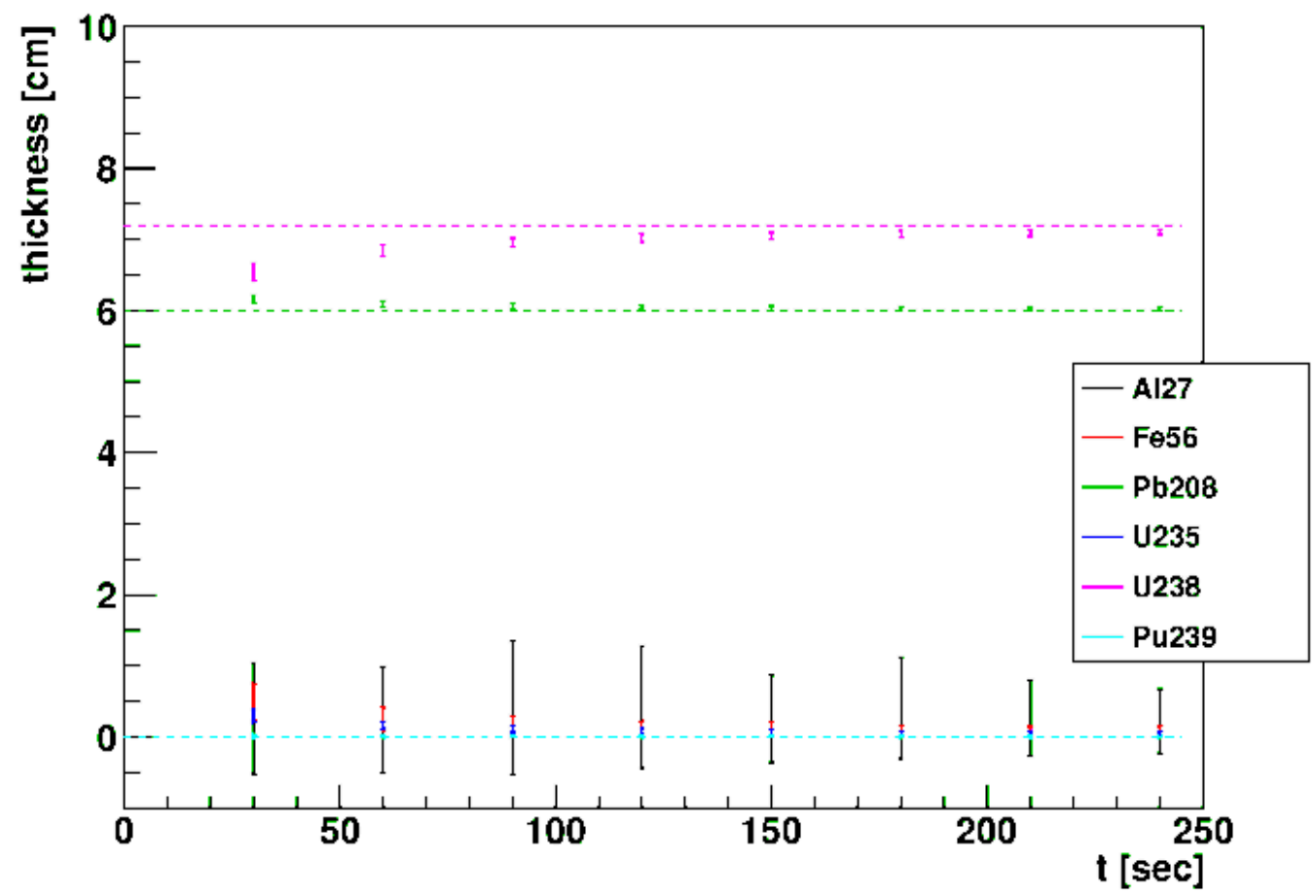

Figure 4-10: Results of the target composition reconstruction simulation using the shape of the photoneutron cross section as a function of gamma-ray energy and integrating the neutrons from $2 \mathrm{MeV}$ and up, with $500 \mathrm{keV}$ per tagger channel. The top figure uses the cross section shape as a function of gamma-ray energy (1D approach) only, whereas the bottom figure includes the variation of the cross section with outgoing neutron energy as well (2D approach). 
We examined the effect of the photon tagging resolution (i.e. the number of tagging spectrometer focal plane channels) on the target composition reconstruction capability. In the simple limiting case of very poor tagging resolution, if the number of tagger channels is smaller than the number of materials in the database, then the one dimensional fits using neutron yield versus $E_{\gamma}$ cannot converge. There is no unique solution. There is also the practical issue of rate in individual tagger channels that requires the use of fairly small channels (100 to $500 \mathrm{keV}$ width in tagged energy per channel). As the number of channels increases, the amount of information available in principle also increases, but at a statistical cost given the reduction in counts detected in any given channel. An optimum balance should be struck among several factors, including the potential information content related to the fineness of the energy bins, the tagging rate per channel, and the counting statistics in any given bin. To begin studying this, we varied the size of the tagger channels. The results with $200 \mathrm{keV}$ wide energy channels spanning a tagged photon range from 6 to $18 \mathrm{MeV}$ are shown in Figure 4-11. There is not much difference between this case and the $500-\mathrm{keV}$ channel width case, except that the fitting routine has a harder time converging on the correct target thickness composition at shorter dwell times. Presumably, though, as more materials are added to the database, the additional information available from smaller gamma-ray energy bins, and hence a smoother variation in the measured yields, will allow for more precise determination of the target composition. 
Tagger $\Delta \mathrm{E}=200.0 \mathrm{keV} / \mathrm{chan}$

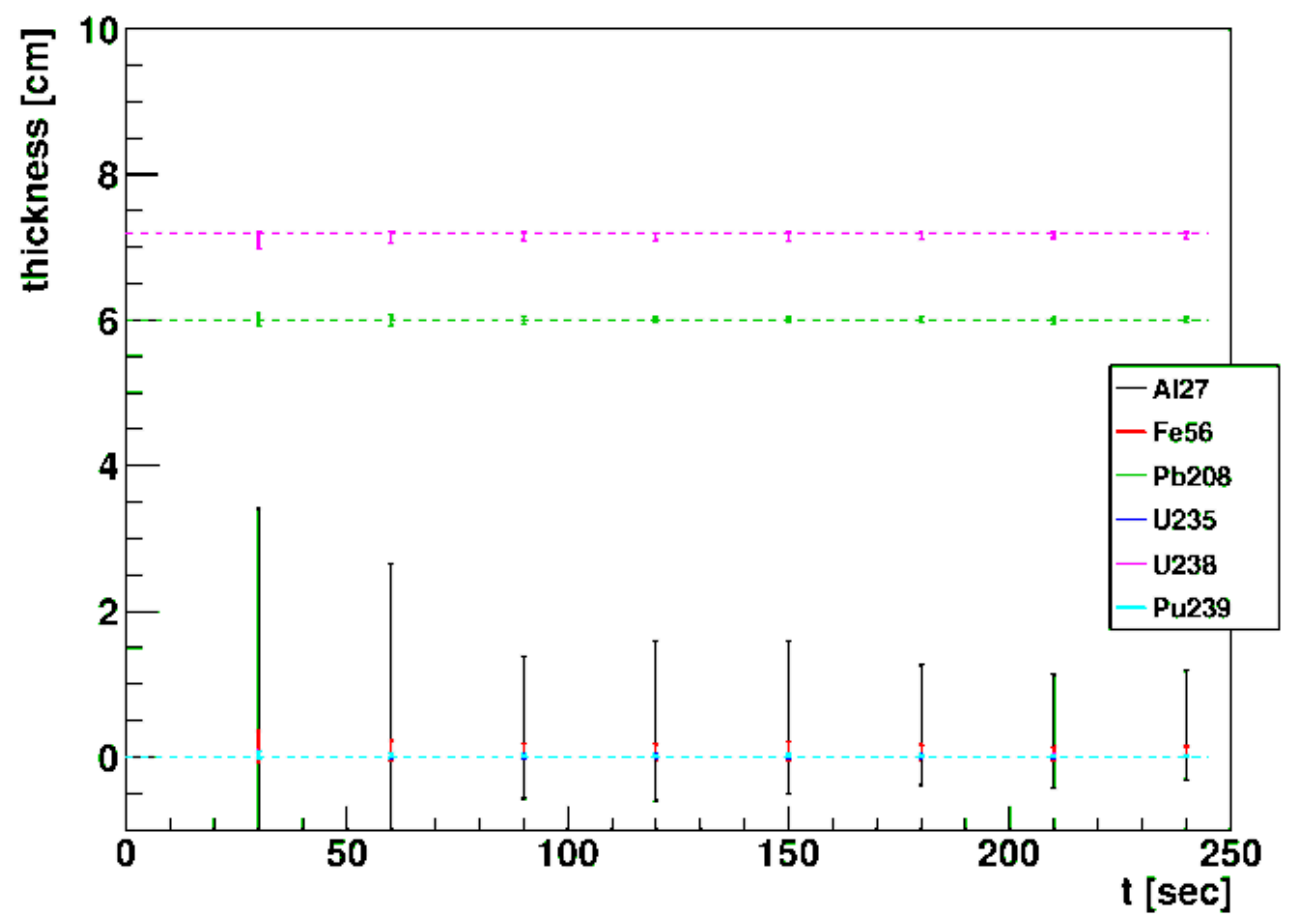

Tagger $\Delta \mathrm{E}=200.0 \mathrm{keV} / \mathrm{chan}$

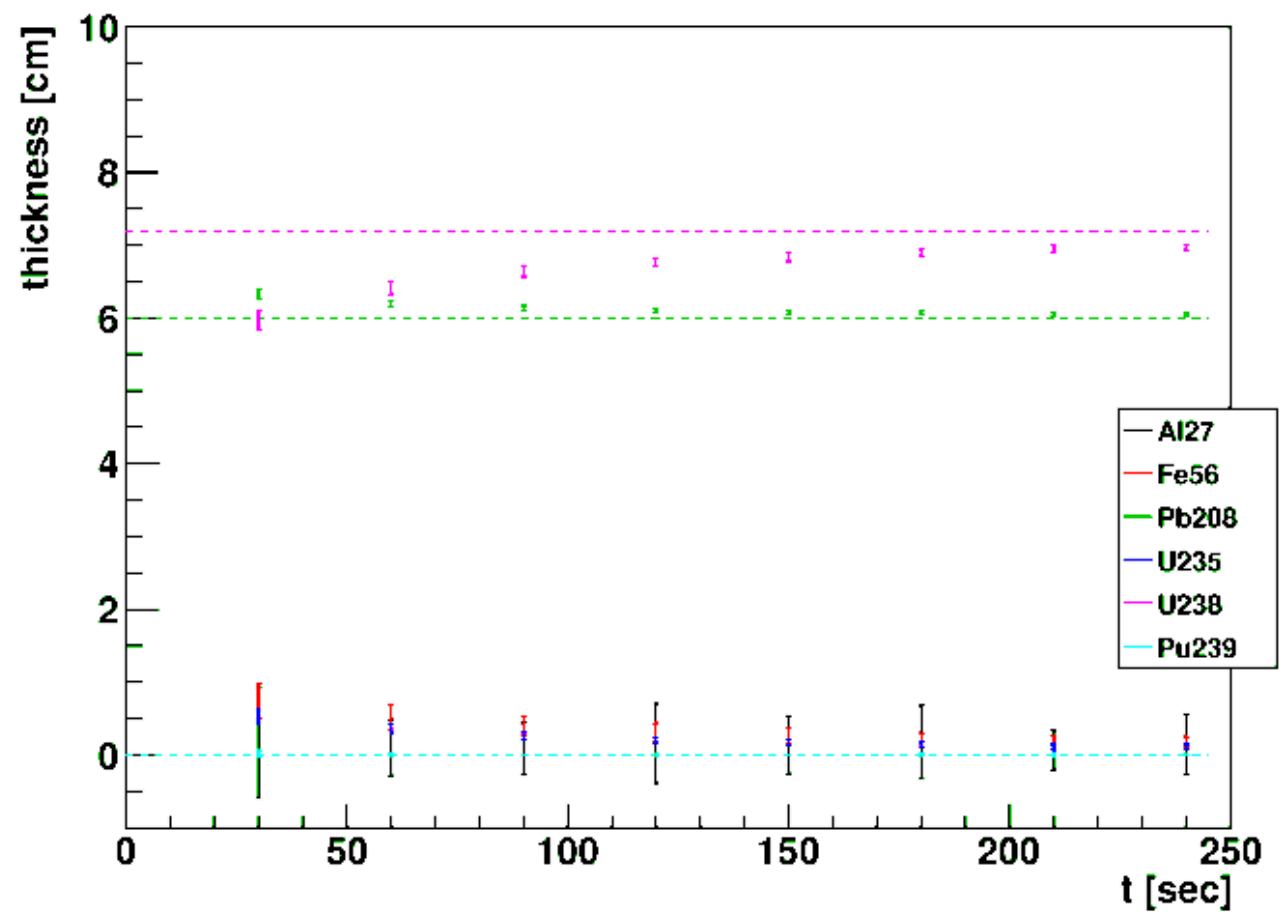

Figure 4-11: Same as Figure 4-10, but assuming a tagged-photon energy width per channel of 200 keV rather than $500 \mathrm{keV}$. 


\section{Photon Tagging: Beamline Facility Infrastructure}

\subsection{Overview}

The photon tagging technique has been used routinely in its various forms to provide quasimonochromatic photons for absolute photonuclear cross section measurements. Various implementations have involved the detection of Compton electrons from a backscattered electron beam [Pascale 1985], the tagging of positron annihilation in flight [Argan 1984], and the tagging of bremsstrahlung beams [Detemple 1992, Terasawa 1986, Bianchi 1992, Anthony 1991, Sober 2000]. This section describes a notional system utilizing tagged bremsstrahlung beams to produce tagged photons in the 10's of MeV range. We describe the basic bremsstrahlung tagging technique, discuss the methods by which one can make very high precision cross section measurements, provide an example of how such a facility will greatly improve a particular measurement, and discuss the design of an accelerator which would enable such a facility.

\subsection{Measurement of absolute photonuclear cross sections with the photon tagging technique}

Here, we discuss in general terms the procedures for performing high precision measurements of photonuclear reaction in the 10 's of $\mathrm{MeV}$ photon energy range where the giant dipole resonance is the dominant excitation mechanism in most nuclei. The motivation for this discussion is to examine experimental considerations relevant both to photonuclear active interrogation (where the range of energies from 5 to $20 \mathrm{MeV}$ may be useful for discriminating fissile from benign cargo materials) and to collection of nuclear data supporting future threat reduction modeling. We note that much of this discussion, and many of the illustrative examples, come from the experience of the Jefferson Laboratory PrimEx Collaboration, which successfully measured an absolute photonuclear cross section with a $1 \%$ contribution of the photon flux to the overall error bar [Teymurazyan 2006]. The primary equipment in such a system includes: (1) a high duty factor (10\% or greater) electron accelerator in the 10 's of $\mathrm{MeV}$ energy range, (2) a photon tagging system; (3) the physics target of interest; (4) a pair spectrometer for online monitoring of photon flux; (5) the experiment specific detection equipment - detectors for neutrons, photons, fission fragments, etc.

We propose to utilize the mature bremsstrahlung photon tagging technique to measure the energy and time information of incident photons in real photon induced reactions. The electron beam of initial energy $E_{0}$ is incident upon a thin (e.g. $10^{-3}$ radation length) bremsstrahlung radiator foil. The electron loses energy in the electromagnetic field of the nucleus and in the process emits an energetic photon. The number of photons with energies in the interval $k-k+d k$ is proportional to the $\mathrm{Z}^{2}$ of the radiator and is inversely proportional to the energy $k$ of the photons [Bethe 1934]. Due to the relatively small mass of the electron, the recoil energy transferred to the nucleus is negligible, and the bremsstrahlung photon energy can be written as:

$$
E_{\gamma}=E_{0}-E_{e}
$$

where $E_{\gamma}$ is the energy of the bremsstrahlung photon and $E_{e}$ is the energy of the secondary

electron. Since the energy $E_{0}$ of the electron incident on the radiator is determined by the 
accelerator, one can determine the energy of the photon by measuring the energy of the post-bremsstrahlung electron.

The main components of a photon tagger are a thin bremsstrahlung radiator, a dipole magnet, and plastic scintillator detectors to measure post bremsstrahlung electrons. In an optimal design such as that of the Jefferson Laboratory Hall B photon tagger [Sober 2000], the post bremsstrahlung detectors on the focal plane of the tagger consist of two rows of plastic scintillator hodoscopes, "E" - and "T" - counters (energy and timing counters). If we assume one wishes to bend a $50 \mathrm{MeV}$ electron beam down $45^{\circ}$ into the beam dump, a 0.12 Tesla $\bullet$ meter magnet is required. In the discussion that follows, it will be assumed that such a system is available.

The photons produced in the radiator continue essentially straight ahead through the tagger, toward the target further downstream in the experimental hall. Depending on the particular experiment, a collimator in conjunction with a sweep magnet can be positioned just downstream of the tagger and centered on the photon beam line in order to further define the photon beam. Post bremsstrahlung electrons are separated from the photons by the tagger dipole magnet. The field setting of the magnet is adjusted to the incident beam energy to allow full energy electrons which do not interact in the radiator to be transported into a shielded beam dump which is typically below the floor of the experimental hall for shielding purposes.

The energy-degraded electrons are detected in the E- and T-counters that lie along a focal plane downstream from the straight edge of the magnet. For continuous energy coverage, the E counters optimally make up a plane of overlapping scintillators and can be designed to have a variable width to cover approximately constant energy bins. Using the overlapping nature of the E-counters, the number of energy channels can exceed the number of detectors by implementing a software coincidence between adjacent E-counters.

In order to properly associate a tagged electron with a related downstream event, a row of T counters located directly under the row of E counters can provide timing resolutions of 300 ps or better. Such resolutions also enable high precision time of flight information. Each detector is a plastic scintillator read out, preferably with a photomultiplier tube at each end of each scintillator. The T counters should have overlapping acceptances to ensure that there are no inter-counter gaps. As in the case of the E counters, the overlapping design of the T-counters gives rise to multiple non-overlapping $\mathrm{T}$-channels which are defined through a software coincidence for two adjacent detectors. The widths of the individual T counters can be made variable to compensate for the $1 / k$ behavior of the bremsstrahlung cross section such that there is the same counting rate for each detector within the same group. The total tagging rate can be as high as 10's of MHz for the whole focal plane.

In general, the use of the photon tagging technique provides at least two significant advantages over experiments involving continuous spectrum bremsstrahlung beams. First, the quasimonochromatic nature of the tagged photon beam enables the unambiguous measurement of the energy dependence of processes of interest. Second, the precise determination of the tagged photon flux on the experimental target is also enabled by the tagger. This more accurate 
knowledge and control of the photon beam energy and the luminosity enables a greater control over systematic errors.

The experimental cross section for a photonuclear reaction (e.g. $(\gamma, X))$ is given by:

$$
\frac{d \sigma}{d \Omega}=\frac{d Y_{X}^{\text {tagged }}}{N_{\gamma}^{\text {tagged }} \cdot \varepsilon \cdot t \cdot d \Omega}
$$

where $d \Omega$ is the element of solid angle of the detector for particle $X, d Y_{X}{ }^{\text {tagged }}$ is the yield of tagged $X$ 's within solid angle $d \Omega, t$ is the target thickness, $\varepsilon$ is a factor accounting for geometrical acceptance and energy dependent detection efficiency, and $N_{\gamma}^{\text {tagged }}$ is the number of tagged photons on the target. As can be seen from Equation 2, the normalization of the cross section directly depends on knowing the tagged photon flux on the target. The number of tagged photons is not equal to the number of hits recorded by the tagging counters because of a number of effects:

1. events in which a bremsstrahlung photon is produced and then absorbed before reaching the target;

2. Møller scattering events in the bremsstrahlung radiator which produce an electron in the tagging counters without an accompanying photon;

3. extra hits registered in the tagging counters due to room background.

To minimize the absorption of photons before they reach the target, the bremsstrahlung beam is best designed so that it travels in vacuum as close as possible to the target. The Møller scattering events are known to affect the tagging rate at the level of a few percent. The impact of the room background on the tagging rates of runs with various electron beam intensities should be continuously monitored.

The combination of these effects can be measured in a calibration run by removing the target and placing a photon detector (typically a $\mathrm{NaI}(\mathrm{Tl})$ detector for photons of 10 's of $\mathrm{MeV}$ ) directly in the photon beam. Assuming that this detector is $100 \%$ efficient in detecting photons in the energy range relevant for the experiment, the ratio of Tagger- $\mathrm{NaI}(\mathrm{Tl})$ coincidences to the number of tagger hits, the so called absolute tagging ratio, is then recorded:

$$
R_{\text {absolute }}^{i}=\frac{N_{\gamma \cdot e^{i}}^{\mathrm{NaI}}}{N_{e}^{i}}
$$

where $N_{\gamma \cdot e^{i}}^{\mathrm{NaI}}$ is the number of photons registered by the $\mathrm{NaI}(\mathrm{Tl})$ detector in coincidence with a particular tagging counter and $N_{e}^{i}$ is the number of electrons registered in tagging counter $i$. Knowing this ratio, one can determine the tagged photon flux in the data taking run by counting the number of post bremsstrahlung electrons in the tagging counters:

$$
\left.N_{\gamma}^{\text {tagged }}\right|_{\text {meas }}=\left.N_{e}\right|_{\text {meas }} \times R_{\text {absolute }} \text {. }
$$

The use of the NaI detector to calibrate the number of tagged photons per electron in the tagger provides an absolute normalization of the tagged photon flux incident on the target. However, these measurements can be performed only at intervals interspersed with data collection. Also in 
the calibration run, the rate of the NaI detector is limited, and therefore, the tagging ratio can only be measured at a rate which is reduced by a factor of about one thousand as compared to the data taking run. Thus any rate and time dependence in the tagging efficiency must be carefully considered and corrected for in applying the NaI-based tagging efficiency normalization.

A pair production luminosity monitor would enable one to measure the relative tagged photon flux over a range of all relevant intensities, and operate continuously throughout the data collection. The pair spectrometer (a notional schematic design is shown in Figure 5-1) could use an appropriate target as a converter to measure the ratio of the number of $\gamma+A \rightarrow A+e^{+}+e^{-}$ reactions in coincidence with a tagging signal to the number of hits in the tagging counters.

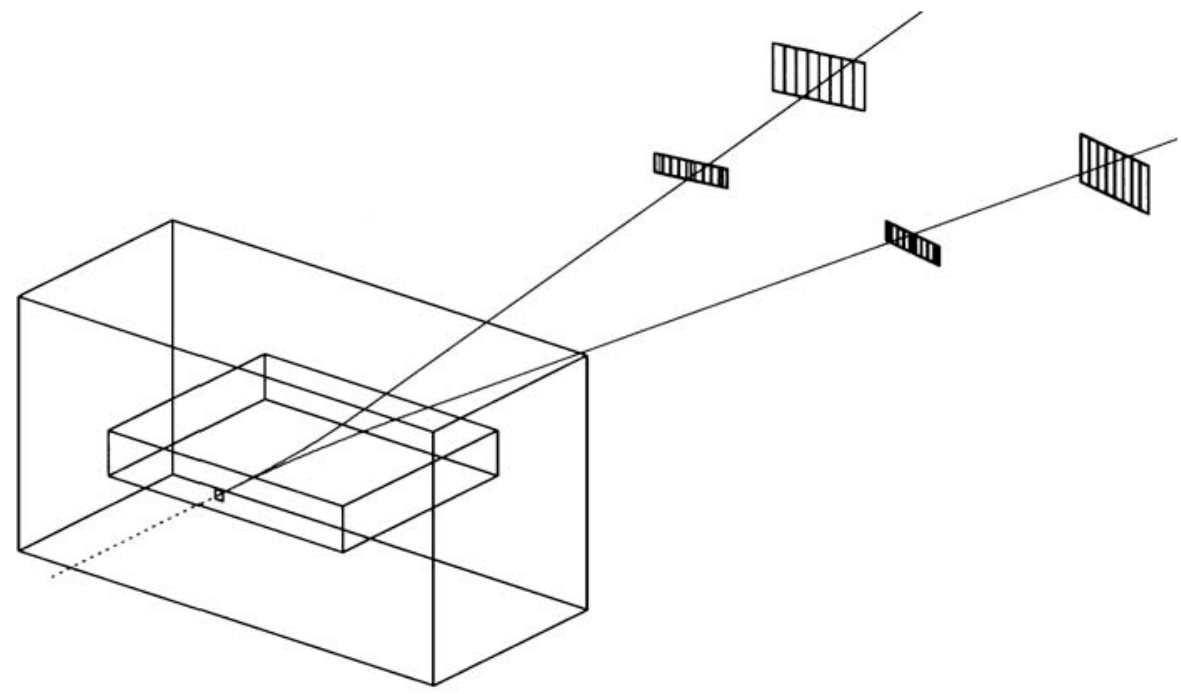

Figure 5-1. Schematic layout of a notional pair spectrometer for continuous relative flux normalization of tagged photons. Each arm consists of eight continuous plastic scintillator hodoscopes in each row. The tagged photon beam enters the spectrometer from the lower-left. Pair production yields secondary electrons and positrons which are split into spatially-separated beams by a magnetic field.

The pair spectrometer enables one to measure a relative tagging ratio:

$$
R_{\text {relative }}^{i}=\frac{N_{e^{+} e^{-} \bullet e^{i}}^{P S}}{N_{e}^{i}}
$$

where in analogy with Eq. 3, $N_{e^{+} e^{-} \bullet e^{i}}^{P S}$ is the number of electron-positron pairs in coincidence with a given tagging counter, $i$, and $N_{e}^{i}$ is the number of electrons registered in the $i$ 'th tagging counter. While this is a relative number, its absolute normalization can be determined with the NaI detector.

The advantages of the pair spectrometer are that it can operate over the entire range of intensities of both the flux calibration with the $\mathrm{NaI}$ detector and the production data taking runs, and has a smooth, relatively flat acceptance in $E_{\gamma}$ covering the entire tagging range. If the pair production target is the same as the physics target for a high-precision cross section measurement, the segmentation of the pair spectrometer detectors would be driven by the thickness of this target. 
Alternatively, in an interrogation scenario one may place the pair spectrometer upstream of the physics target and equip it with a separate, thin pair production target to have more flexibility. This is the preferred scenario for this notional facility. In general, the efficiency of the pair spectrometer for tagging photons depends upon the pair production cross section and the momentum acceptance of the electron and positron detectors. Efficiencies of a fraction of $1 \%$ are suitable for relative flux monitoring, so this target may be very thin.

To reduce the data acquisition rates, the primary trigger is best not induced by the tagged photons, but by the photonuclear detector ( $X$ detector in the above nomenclature), which means there are possible events in the data which are induced by untagged photons. These events are excluded from consideration. In the yield one counts only $X$ events which are tagged as true events. The $N^{\text {tagged }}$ in the denominator of Eq. 2 has to be counted consistently with the way $Y_{X}^{\text {tagged }}$ is determined. This means that if events are discarded from the yield calculation, they should not be considered when calculating the photon flux either, and vice versa. Further, triggering on the $X$ detector signal in data collection as opposed to the tagger has implications in the determination of the number of post-bremsstrahlung electrons in the tagger. This will be discussed below.

\subsection{Absolute Flux Normalization}

In the calibration runs designed to measure the absolute tagging ratios, the experimental target is retracted and a NaI detector is placed in the path of the photon beam. To avoid radiation damage to the NaI detector, the electron beam intensity is lowered considerably to 10's of pAmperes, and a thinner bremsstrahlung radiator may be used to reduce the photon rates. For such runs, the low intensity of calibration runs enables the use of the Tagger Master OR (MOR) signal as the data acquisition trigger. The MOR signal is formed from the logical OR of the pulses from all or any of the T counters. Using the MOR trigger enables one to directly count the number of electrons that hit the tagging counters. However, due to the reduced intensity of the primary beam, even a slight variation in room background can have a significant negative effect on the tagging ratios. Thus periodic measurements are necessary to ensure a stable, reproducible result.

Absolute tagging ratios are then defined for each of the T counters as in Eq. (3). A number of measurements have been performed to investigate the robustness of this procedure and are outlined in the following sections. To illustrate that $\sim 1 \%$ photonuclear cross section measurements are possible, we summarize the experiences of the Jefferson Laboratory PrimEx Collaboration in this regard.

\subsection{New techniques for high precision measurements}

The analysis of such experiments in the context of bremsstrahlung photon tagging was summarized in [Owens 1990]. Since then, a number of developments have made possible significant improvements in the implementation of this technique. Here, we describe the steps which can limit the systematic uncertainty in the absolute photon flux to $1 \%$. They include an absolute flux calibration at low intensity with a NaI detector, online relative flux monitoring with a pair spectrometer, and the use of multi-hit time to digital converters for post bremsstrahlung electron counting during production data runs. 


\subsubsection{Nal - Tagger coincidence in calibration runs}

In the tagging ratio measurements, because the tagger rates will be low, the time difference between the tagger and the NaI detector will form a coincidence peak with a flat background. Figure 5-2 shows a typical coincidence spectrum (again, example plots are from the PrimEx experiment). Note that the signal to background ratio is better than 10000:1, and thus the determination of the number of coincidences is quite insensitive to the accuracy of the background estimation procedure. While the background is not uniform on either side of the coincidence peak, due to the large signal to noise ratio in the plot, taking the background on the left side of the coincidence peak to be flat and uniform and using this to estimate the background introduces negligible error.

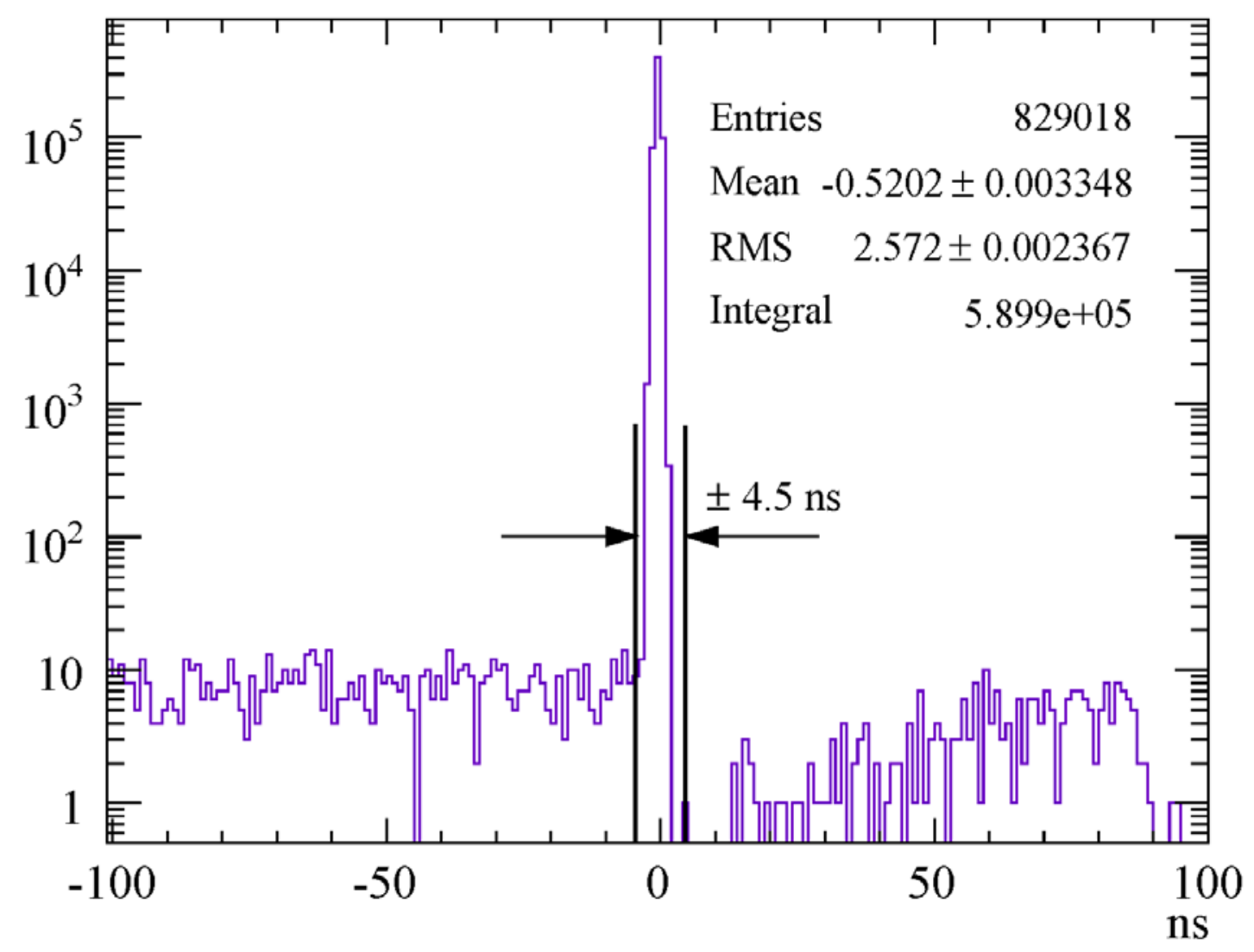

Figure 5-2. Distribution of time differences for events reconstructed for tagger and photon detector (in this case a lead glass detector) showing the \pm 4.5 -ns timing window for coincidence events. Data are from the Jefferson Laboratory PrimEx experiment.

\subsubsection{Stability of absolute tagging ratios}

As previously mentioned, technical limitations of the NaI detector will limit the absolute tagging ratio measurements to beam intensities which are $\sim 10^{3}$ times lower than the intensity of a regular data collection measurement. Since the flux calibration and data collection runs will be 
performed under different beam conditions, it is important to demonstrate that the tagging ratios obtained at beam intensities of $\sim 80 \mathrm{pA}$ are valid when applied to the data collected at the high beam intensities of about 80 to $130 \mathrm{nA}$. One means to investigate this involves normalization runs with various beam intensities which the NaI detector can accommodate (40 - $120 \mathrm{pA})$. While this study will involve only a limited range of intensities, a more complete answer to the question of intensity dependence of tagging ratios can be found by looking at relative tagging ratios where the beam intensity can be varied over a much larger range, from 0 to 100-150 nA. This will be discussed later.

In addition to these rate studies, in each measurement performed, NaI calibration runs can be performed throughout the data collection to ensure stability of the tagging ratio measurements.

\subsection{Normalization of the production data runs}

As indicated in Eq. 4, a key element in measuring the tagged photon flux is the counting of the post-bremsstrahlung electrons in the tagger. For most tagged photon experiments, photons are produced at a rate far greater than is practical for direct counting by the data acquisition system. The exception to this is the NaI calibration runs where, as mentioned above, the rates are lower by a factor of one thousand.

The traditional technique to measure normalization involves hardware scalers that are used to count the number of hits in a particular tagging counter. Scalers have the advantage of being able to count virtually all the hits from a detector. Also, using scalers to measure the detector rates can automatically account for beam-trips, i.e. uncontrolled beam intensity drops or spikes, provided that the scalers also count signals from a beam-related source. However, the triggering scheme proposed here makes the hardware scaler method unattractive exactly due to the fact that scalers would count all the hits in the tagging counters.

The primary trigger for the notional data collection scheme is formed by a coincidence of signals from the $X$ detector and the tagger. When the appropriate signal appears in the $X$ detector, and there is a signal available from the tagger, a trigger signal will be formed which instructs the data acquisition system to read out all the channels that have non-zero information. It is more efficient to use the $X$ detector-tagger coincidence as a primary physics trigger since using the tagger signal alone would flood the data acquisition due to the high rate of tagging counter signals, most of which represent photons which passed through the target without producing an event of interest.

Our notional design incorporates a data acquisition system based upon multi-hit time to digital converters (TDCs). The LeCroy LRS1877 TDC has been successfully implemented in the past. It has a maximum range of $32 \mu \mathrm{s}$, double pulse resolution of $\sim 20 \mathrm{~ns}$, and the capability of storing up to 16 hits per trigger event per channel in a LIFO (Last In First Out) mode. The range of the TDC and the LIFO limit are programmable. The capabilities of these TDCs allows for significant improvement in the analysis techniques of tagged photon measurements, e.g. those described in [Owens 1990], which considered the analysis of tagged photon experiments using single hit TDC's. 
Since only a timing coincidence is required between the $X$ detector and tagger MOR (OR of T counters) signals to form a trigger, there are three scenarios for losing tagged event yield due to the TDC dead-time -- (double pulse resolution) and LIFO limit:

1. An entire event is lost due to TDC dead-time, i.e., there was no signal from tagging counters to form a coincidence with the $\mathrm{X}$ detector signal but the data acquisition system is ready to take data. From the standpoint of photon flux calculation, this case is very similar to the situation where the data acquisition system is busy reading out data and is not accepting any triggers.

2. A photon is lost due to TDC dead-time but a coincidence of another photon from a different tagging counter with a $\mathrm{X}$ signal forms a trigger and an event is read out.

3. A photon producing an $\mathrm{X}$ event may be lost due to the LIFO limit but the triggering condition might be satisfied by a signal from another tagging counter. As in the previous case, such events will not contribute to the tagged yield.

Consideration of the three situations presented above leads to the conclusion that the photons that are untagged due to TDC dead-time or LIFO limit cannot produce a tagged $X$ event.

For purposes of normalizing a tagged-photon measurement, one is interested only in the number of tagged photons on the target which have the potential to produce a tagged event. The tagged $X$ yield automatically includes the effects of TDC intrinsic dead-time, LIFO limit and the data acquisition readout/dead-time. Hence, it is not necessary to correct for the number of tagged photons lost due to these effects.

The rate of tagged photons can be determined from the timing information, recorded by tagging counters, via sampling of the number of hits for a small fraction of the time. An assumption is then made that these samples are representative of the detectors' rates for the times when no data are recorded. This can be used to extrapolate to all times in order to determine the total number of tagged photons represented by a given data sample. Since one is interested in the number of tagged photons that have the potential to produce a tagged $X$, the timing information from only fully reconstructed hits in the tagger need to be considered. A fully reconstructed hit would require a timing coincidence between the left and right PMTs of a T-counter that are simultaneously in time with a hit in an E-counter. The coincidences between E- and T-counters would also be subject to a geometric matching where the two counters are required to be on an electron trajectory which is consistent with the magnetic optics of the tagger.

The LeCroy 1877 TDCs in this scenario can be operated in common stop mode. A T-counter signal passes through a constant fraction discriminator and would be split into two signals. One signal would start the TDC and the other signal would pass through E-T coincidence/MOR module. Assuming a coincidence between the left and right T counter PMTs and an E counter has occurred, the MOR module would send a signal to the trigger supervisor when any E-T coincidence is obtained. If a signal from the $X$ detector is in coincidence with the MOR signal, the trigger supervisor would send a common stop trigger signal to all electronics involved in the data acquisition. Figure 5-3 and Figure 5-4 show an example of a timing spectrum of hits reconstructed for a single T-counter in the tagger. Note that the abscissa in Figure 5-3 is presented on a log scale. The peak in the timing spectrum at around $100 \mathrm{~ns}$ corresponds to the 
time difference between the two split signals from a single T-counter, i.e., it is associated with the events when this particular T-counter was involved in the trigger. The flat accidental background comes from signals that were not involved in the trigger but were accidental hits recorded due to the common stop/multihit nature of T-counter TDCs.

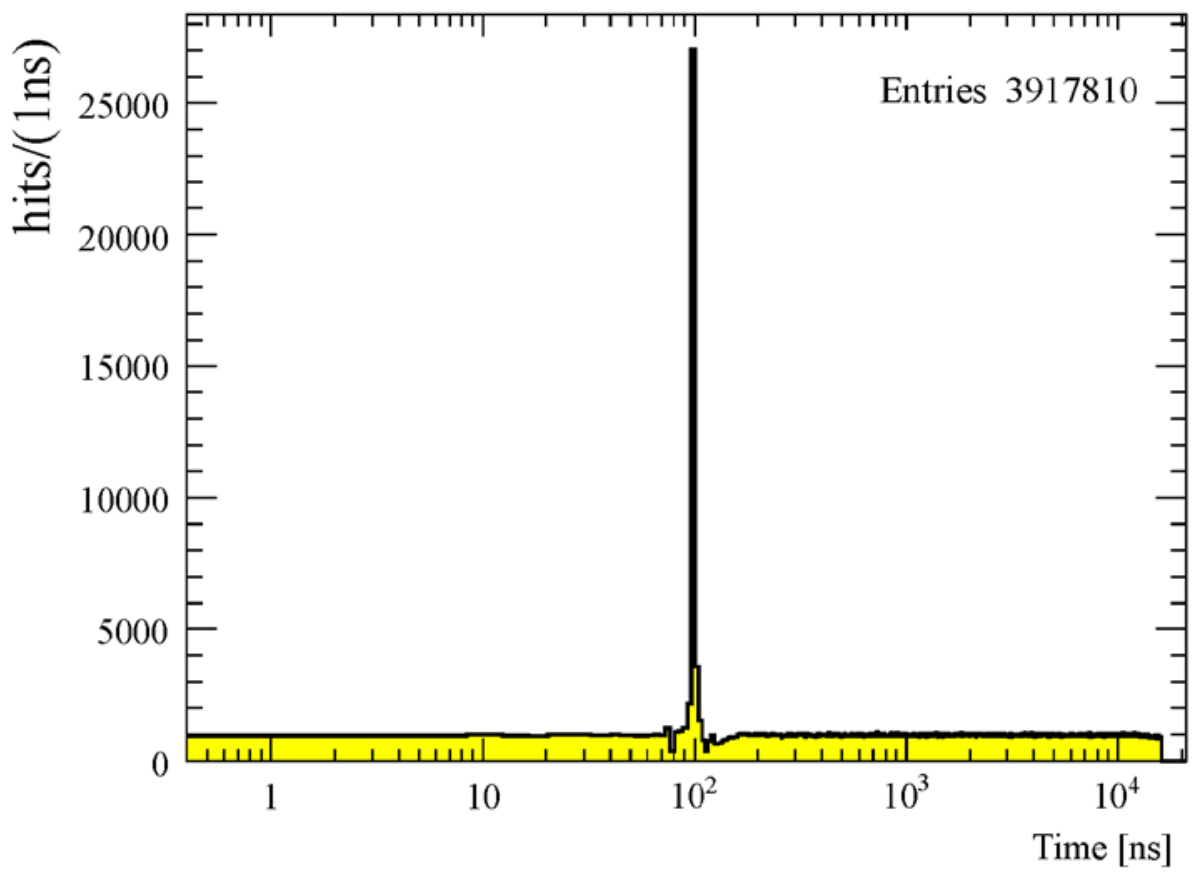

Figure 5-3. Time spectrum of hits reconstructed for a single T-counter. Data from the Jefferson Laboratory PrimEx experiment. 


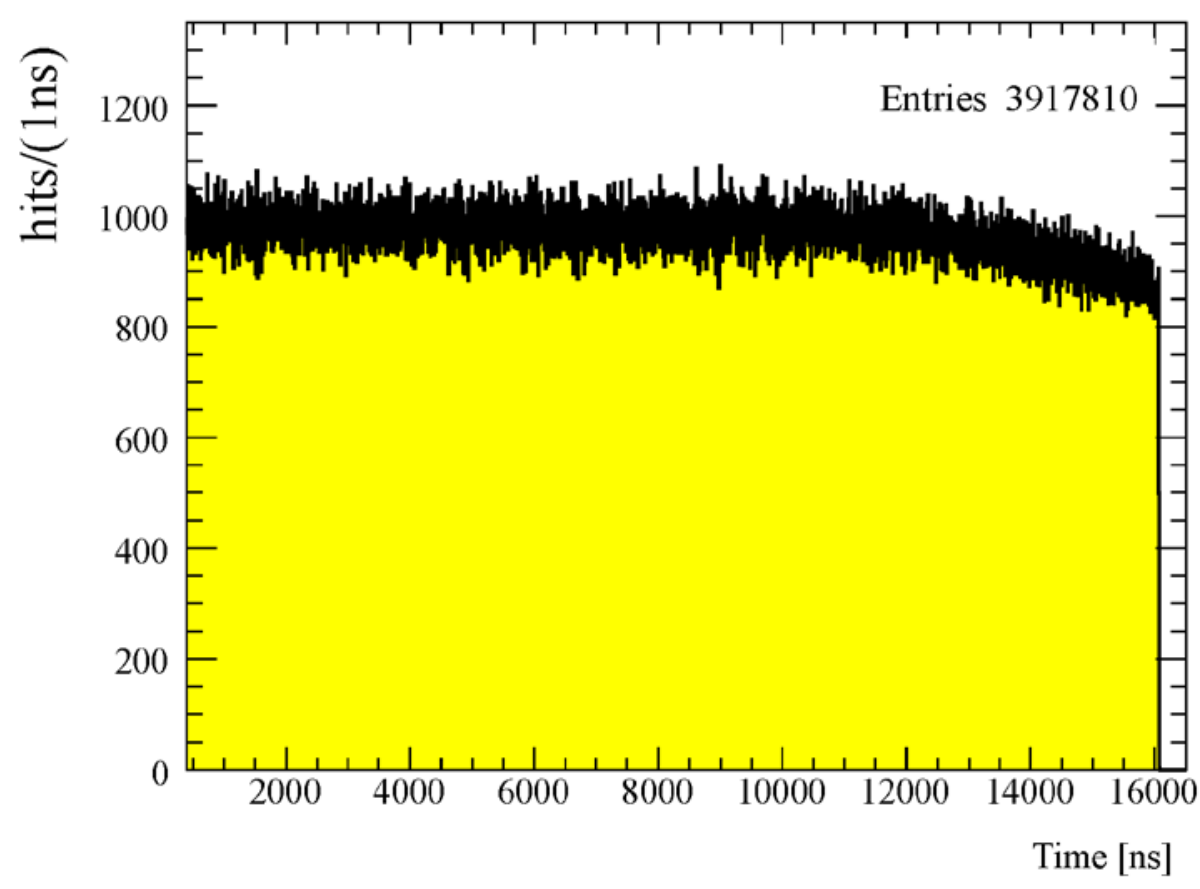

Figure 5-4. A close up of part of the previous plot illustrating the drop off of the number of hits due to the LIFO limit.

One obvious effect seen in Figure 5-4 is that the number of hits trails off on the right side of the spectrum due to the LIFO limit. Since in this case the LeCroy 1877's were used in a common stop mode, earlier times are to the right and later times are to the left in this plot. The LeCroy 1877 TDC will always report the latest hits. Thus when the LIFO fills up, the earlier hits are overwritten by later ones.

\subsubsection{The "out of time" method}

The tagged photon flux at the target can be determined by means of sampling the "out-of-time" (OOT) electron hits in the tagger T-counters. The term "out-of-time" electron refers to any fully reconstructed electron which was not involved in the formation of the trigger signal. The idea is to simply count the number of hits in a particular T-counter within some user defined time window $w$ and divide by the size of the time window. Since even high rate detectors on average tend to have only a few hits per event, it is necessary to integrate over many events to obtain an accurate value for the rate.

When counting hits, it is important to discard those that could be associated with the trigger. Hits which are correlated with the trigger are biased and will artificially increase the calculated rate. The OOT window, $w$, should be defined in such a manner that it does not include the trigger coincidence peak region but can include areas both before and after the trigger peak. One drawback of this rate sampling technique is that it is potentially vulnerable to beam intensity variations, since it will tend to sample more often when the beam intensity is higher. As such, the “clock trigger" method can be implemented as described below. 


\subsubsection{The "clock trigger" method}

To ensure that the calculated rates are not biased by beam intensity variations, a clock trigger, which is completely uncorrelated with the electron beam current, can be implemented in addition to the physics trigger. The clock triggers can be pre-scaled so that the data are dominated by events of physics type that are of interest. Figure 5-5 shows a sample timing distribution for hits reconstructed in a single T-counter recorded with the clock trigger. As in the case of the tagger MOR- $X$ detector coincidence trigger, one can see a depletion of hits due to the LIFO limit starting at around $10 \mu \mathrm{s}$, but the peak characteristic of a beam related trigger is missing.

The same out of time window, $w$, shown in Figure 5-5, is used when calculating the rates with either clock or physics triggers. Here, it was chosen to be $7 \mu$ s for all T-counters spanning the range from 500 to $7500 \mathrm{~ns}$, thus avoiding the coincidence peak in the case of MOR- $X$ detector coincidence trigger and the region affected by the LIFO limit for both triggers.

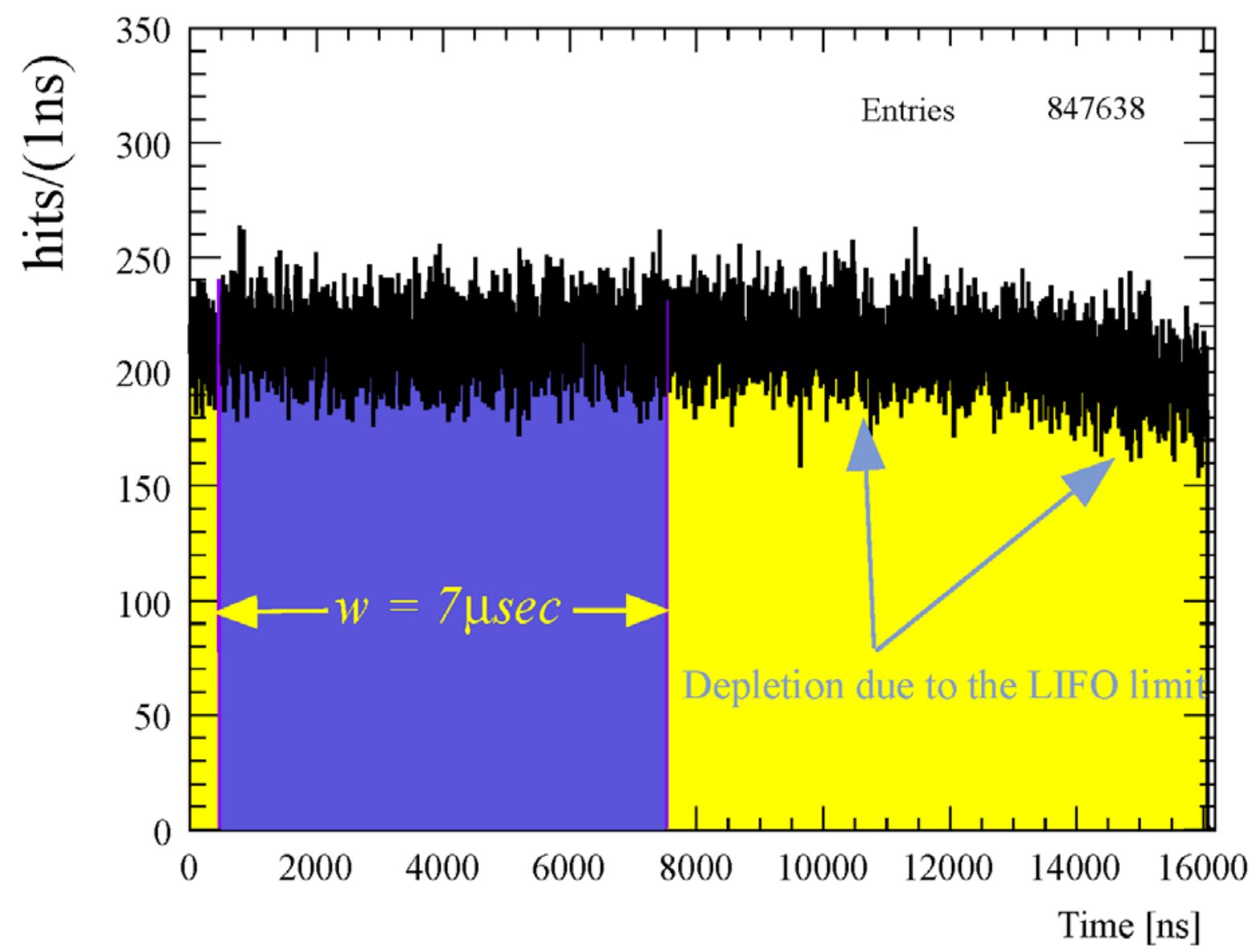

Figure 5-5. Timing spectrum of hits reconstructed for a single T-counter. These data were taken with clock triggers. Data from the Jefferson Laboratory PrimEx experiment.

Following the procedure described above for an electron rate calculation we have: 


$$
r^{i}=\frac{n_{e}^{i}}{w \cdot n_{\text {trigger }}}
$$

where $r^{i}$ is the rate of T-counter $i, n_{e}{ }^{i}$ is the number of hits within the out-of-time window of width $w$, and $n_{\text {trigger }}$ is the number of times the T-counter $i$ could have had a hit, i.e., the number of triggers. Equation 6 assumes Poisson statistics for "out of time” electrons and it assumes constant electron rate per T-counter.

The number of tagged photons $N_{\gamma}{ }^{i}$ in T-channel $i$ can be calculated as:

$$
N_{\gamma}^{i}=N_{e}^{i} \cdot R_{\text {absolute }}^{i}
$$

where $N_{e}{ }^{i}$ is the number of electrons per T-channel $i$ and $R_{\text {absolute }}^{i}$ is the tagging ratio, which is determined via the NaI calibration.

\subsection{Relative photon flux calibration with pair production}

The pair spectrometer is intended for relative in-situ monitoring of the photon flux and is an essential part of any high precision measurement. It uses a target to convert a fraction of the photons into $e^{+} e^{-}$pairs which are deflected in the field of a dipole magnet downstream of the target and are registered in plastic scintillator detectors on both sides of the beam line. The relative tagging ratio for a given T-counter is given by Eq. 5 .

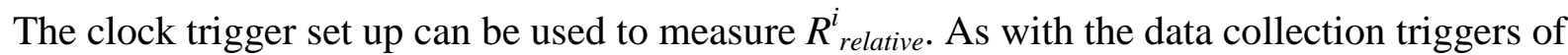
interest, the clock trigger enables one to directly count the number of electrons in the tagging counters and it has the advantage of being insensitive to beam intensity variations.

\subsubsection{PS-tagger coincidence window and background}

The event times reconstructed in both the tagger and the pair spectrometer are randomly distributed in time with respect to the clock trigger. The time difference of hits reconstructed for the tagger and the pair spectrometer gives rise to a coincidence peak and a non-flat, triangularly shaped background. An example timing spectrum is shown in Figure 5-6. 


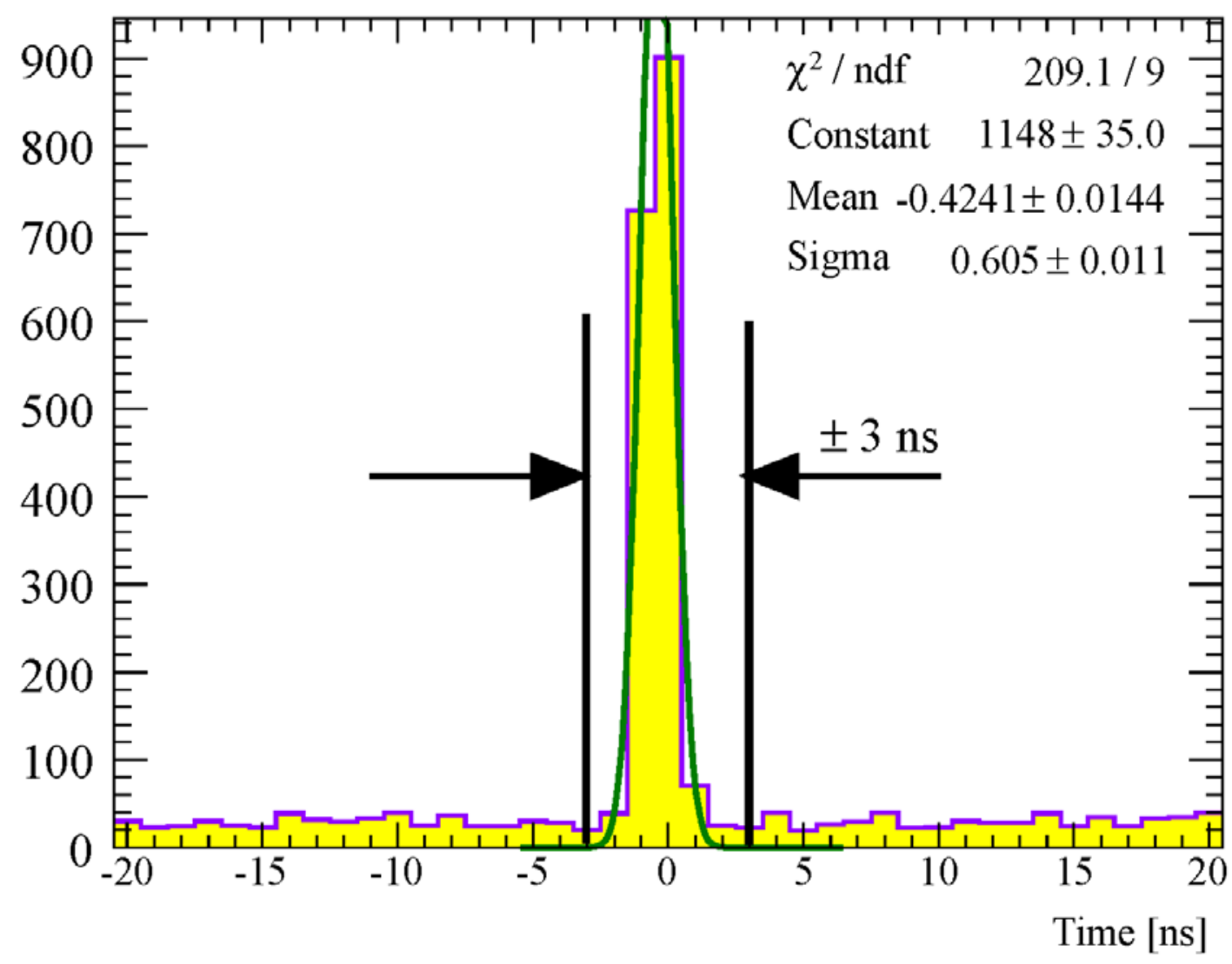

Figure 5-6. Distribution of time differences for events reconstructed in the tagger and pair spectrometer showing the \pm 3.0 ns timing coincidence window. Data from the Jefferson Laboratory PrimEx experiment.

In general, taking the difference of two random distributions, defined over the same interval, results in a triangular shape distribution. This provides an exact background model, which enables one to easily simulate the "background only" part of the spectra.

\subsubsection{Effect of incident electron beam intensity on relative tagging ratios}

In order to justify the use of the absolute normalization of the photon flux obtained at the low electron beam intensities of the NaI flux calibration for the calculation of the number of tagged photons on target, it is important to demonstrate the independence of the $R_{\text {relative }}^{i}$ on the electron beam intensity. The relative tagging ratios, defined by Eq. 5, provide valuable confirmation of this procedure as they can be measured at the low energies of the NaI calibration as well as at high electron beam intensities of the primary data collection.

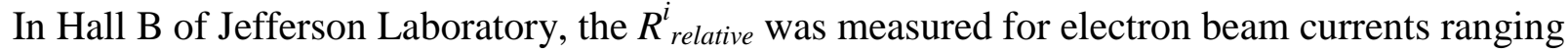
from 0.08 to $100 \mathrm{nA}$. The results for a representative T-counter are shown in Figure 5-7 and Figure 5-8. The data points were fitted with a polynomial and as indicated in the figure, $R_{\text {relative }}^{i}$ is quite independent of the beam intensity. Measurements analogous to these would be made in the notional tagging system outlined here. 


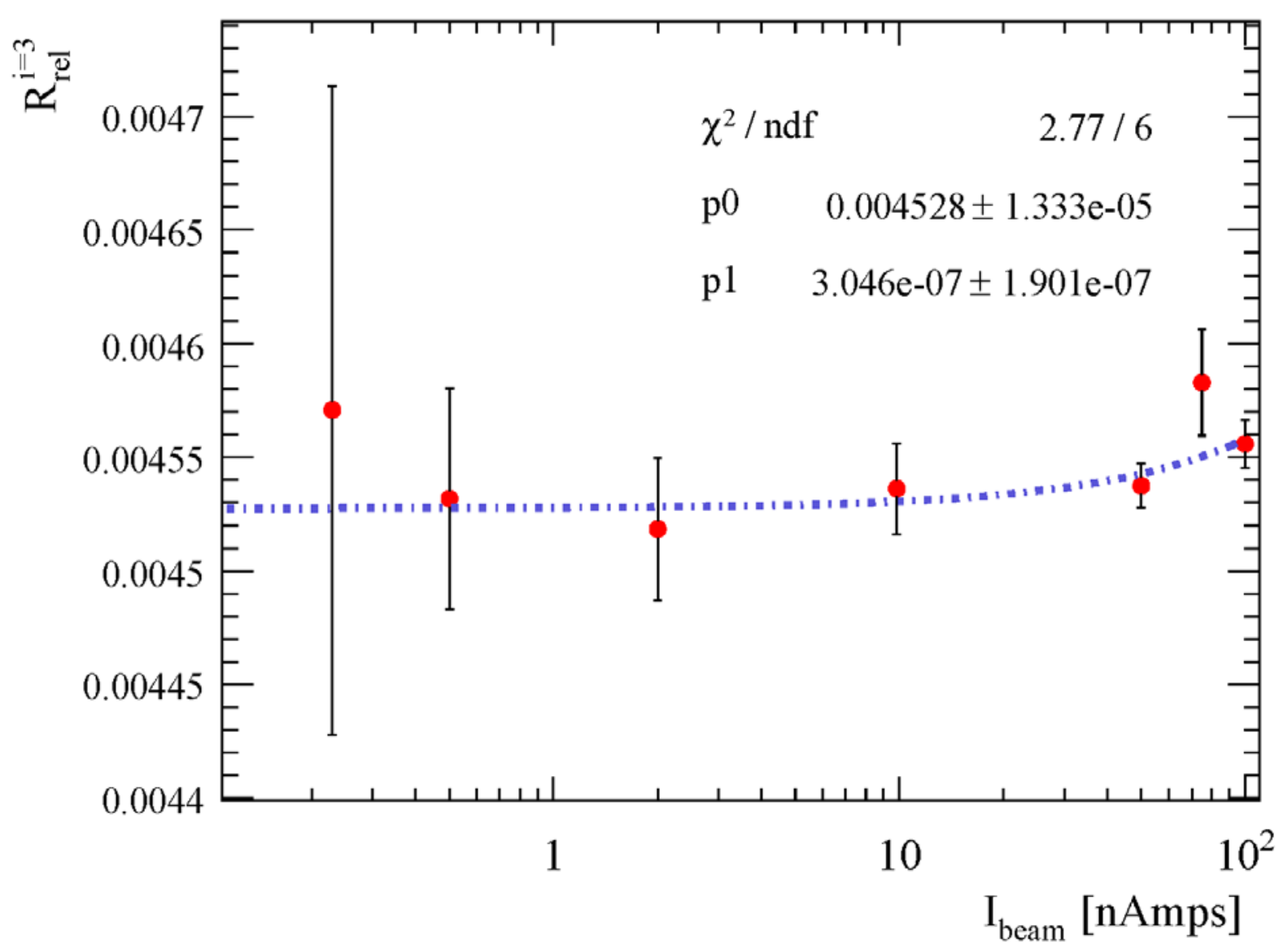

Figure 5-7. Measured $R_{\text {rel }}^{i}$ for T-counter \#3 as a function of nominal electron beam current. Data from the Jefferson Laboratory PrimEx experiment. 


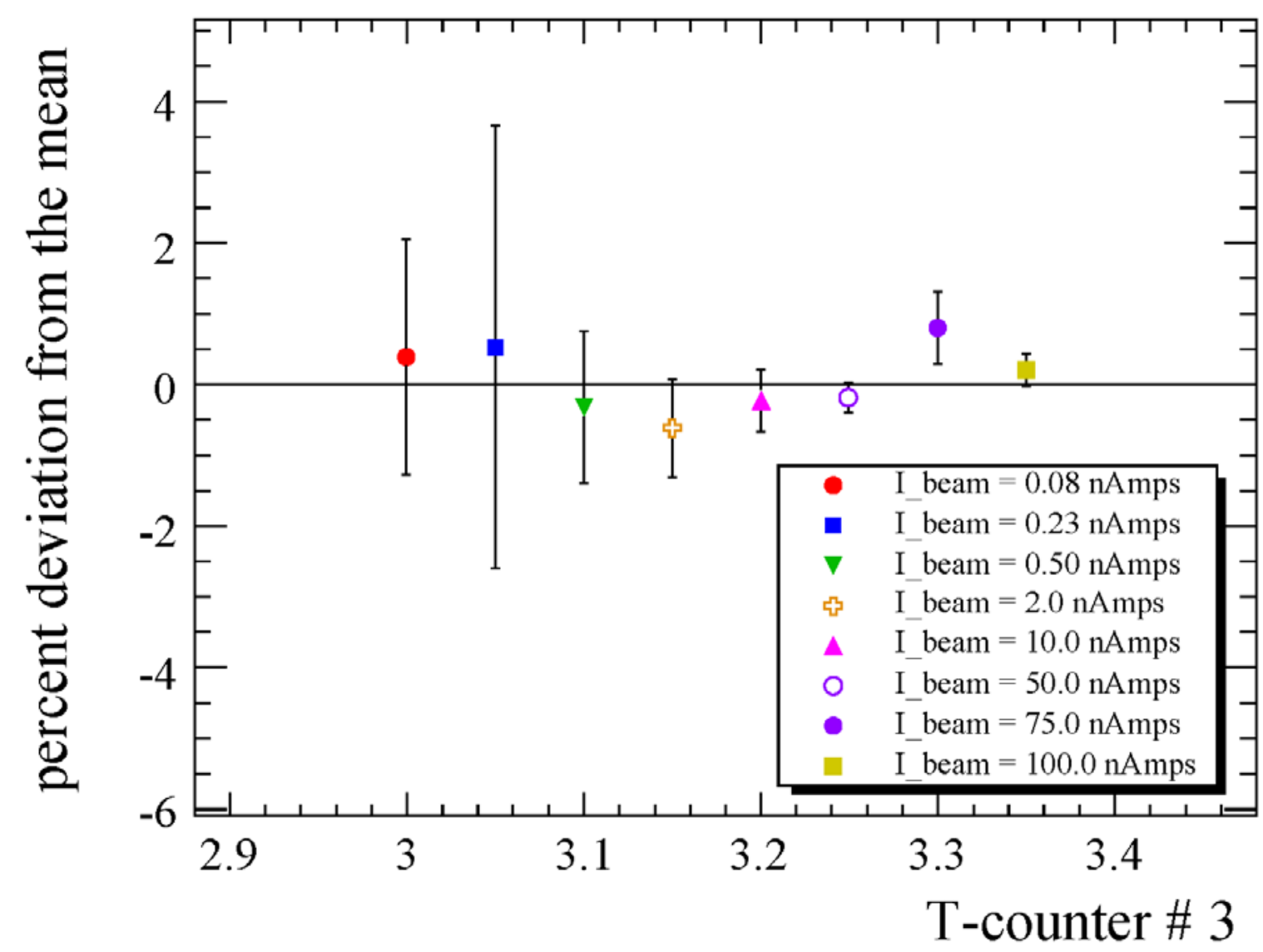

Figure 5-8. The percent deviation from the mean for tagging ratio measurements made at different beam intensities for the first eleven T-counters. Data from the Jefferson Laboratory PrimEx experiment.

\subsubsection{Stability of relative tagging ratios}

The relative tagging ratios have to be not only intensity independent but also stable in time. The time stability of the relative tagging ratios measured by the pair spectrometer justifies the use of a single set of absolute tagging ratios measured by the NaI for the tagged photon flux calculation. To achieve a 1\% tagged photon flux measurement, any deviation from the nominal value of the $R_{\text {relative }}$ has to be carefully investigated and corrected, if possible. In the discussion that follows, the data from eleven T-counters were combined together and treated as one single counter, thus enabling a reduction in the statistical error.

Figure 5-9 shows the time dependence of the $R_{\text {relative }}^{\text {combine }}$ - the combined relative tagging ratio for data taken with a carbon target. The two black solid lines on the graph represent $\pm 1 \%$ deviation from weighted average. One can see that for the last group of runs (run number $>5150$ ), the relative tagging ratio falls off. The deviation is larger than $1 \%$ and indicates that extra care is needed when calculating the photon flux for this group of runs. The point here is that the pair spectrometer provides a powerful diagnostic tool for tagged photon flux normalization. 


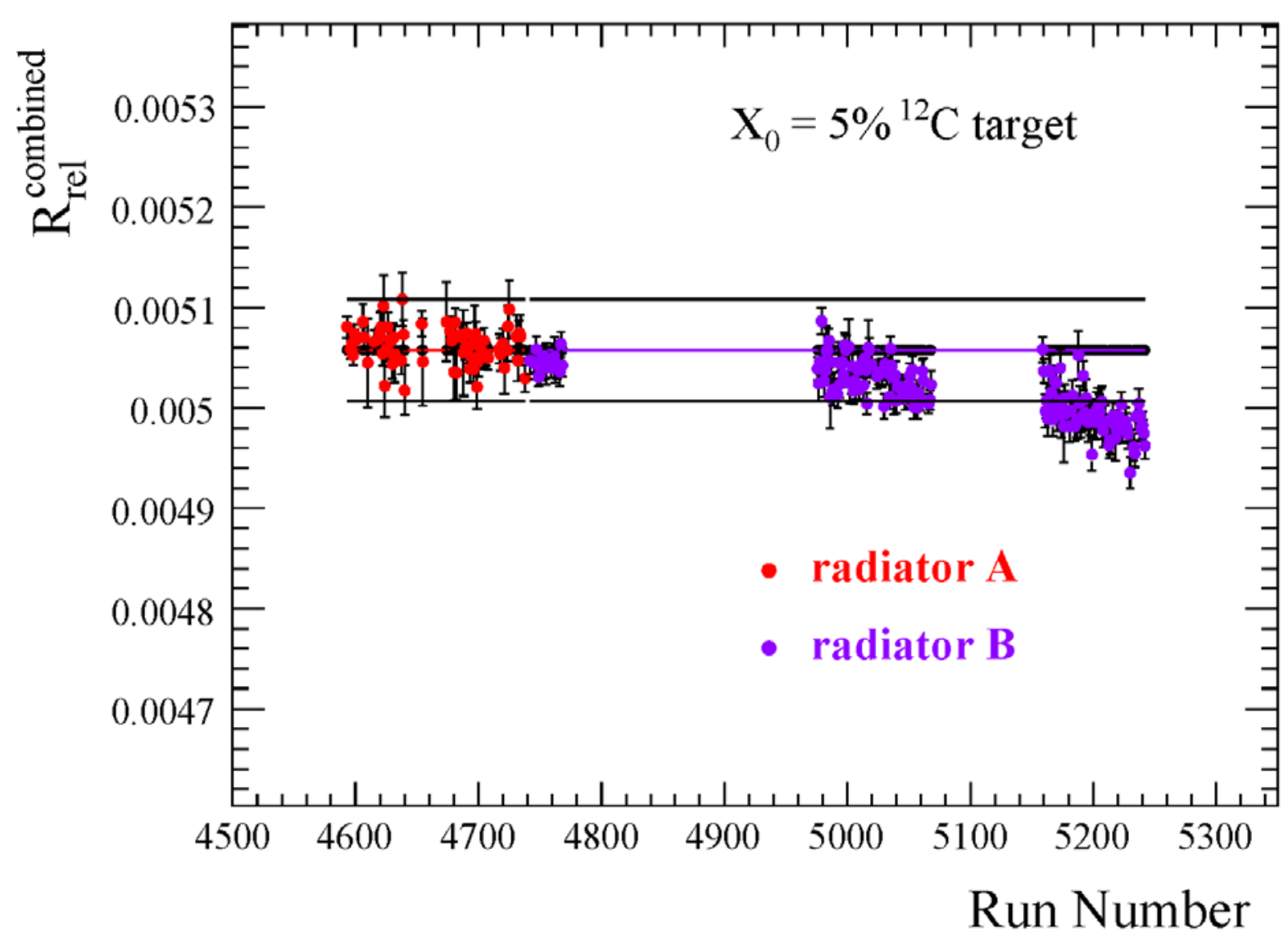

Figure 5-9. Run-to-run stability of $\boldsymbol{R}_{\text {rel }}^{\text {combined }}$ - relative tagging ratio combined for eleven T-counters -- carbon target. Data from the Jefferson Laboratory PrimEx experiment.

Without high duty factor accelerators, coincidence experiments such as those involving photon tagging are impractical. This is because in forming a coincidence, the number of true coincidences is proportional to the beam current, whereas the number of accidental coincidences is proportional to the square of the instantaneous current. For a given average current, the instantaneous rates in standard pulsed (low duty factor) machines is either zero (between pulses) or very high (during the pulse.) Additionally, particle detection "in the flash" when the accelerator is on is problematic due to the high instantaneous background rates. Given these considerations, a CW electron beam is essential for bremsstrahlung photon tagging work.

The availability of a high duty factor electron beam will have multiple applications to ongoing R\&D work, and will open new research avenues. Here, we list a few of the many possible research areas which such an accelerator would enable in the area of photo-induced signatures for the detection of special nuclear materials.

Photon induced signatures for special nuclear materials generally fall into four categories. The basic nuclear de-excitation responses to photon excitation are: 
1. Prompt Gamma Emission. This includes accelerator-stimulated x-ray fluorescence based on the high energy photon excitation of atomic levels, nuclear resonance fluorescence produced by high energy photons, as well as other prompt gamma-producing processes such as photofission and photon-induced nucleon emission leading to nuclear isomers and/or beta-delayed gamma emitters.

2. Prompt Neutron Emission includes $(\gamma, \mathrm{n}),(\gamma, 2 \mathrm{n})$, and ( $\gamma$, fission) processes. Detection and energy spectroscopy of neutrons can be done by neutron time of flight, via proton-recoil pulse height analysis, or by exploiting energy thresholds. These measurements may then be used as a measure of neutron energy distributions and thus as an identification technique.

3. Delayed Neutron Emission. This includes post-fission neutrons from highly excited fission fragments and any beta-delayed or spin-delayed neutron emissions that follow. With few exceptions, only fission fragments emit such neutrons, with one notable exception being ${ }^{17} \mathrm{~N}$, $\mathrm{t}_{1 / 2}=4 \mathrm{~s}$, from the ${ }^{18} \mathrm{O}(\gamma, \mathrm{n})$ reaction. Measurements of these delayed neutrons will include energy spectroscopy for prompt neutrons and temporal spectroscopy, meaning measurements of yield versus time and the extraction of effective lifetimes - photo-induced group parameters, analogous to neutron-induced group parameters. This delayed neutron lifetime analysis has been shown to have potential for use in parent fissile nucleus detection and identification.

4. Delayed Gamma Emission. These processes involve either beta-delayed or isomer-delayed gammas emitted from fission products, from $(\gamma, \mathrm{xn})$ and related processes, and from neutron capture gammas. Temporal and energy spectroscopy, as with delayed neutrons, shows promise for discriminating photo-fissionable material from other materials and discriminating fissile material $\left({ }^{233} \mathrm{U},{ }^{235} \mathrm{U},{ }^{239} \mathrm{Pu}\right)$ from other photo-fissionable material.

\subsection{An illustrative example}

A high duty factor bremsstrahlung photon beam coupled with a photon tagger is ideally suited for measuring the neutron photoproduction cross section as a function of energy. It would allow a wide range of photon energies to be measured at once with excellent (sub MeV) energy resolution and precise absolute flux normalization.

Here, we illustrate the utility of tagged photon beams using an example of a measurement of interest to the National Nuclear Security Agency. In a recent NNSA Funding Opportunity Announcement (Proliferation Detection Research, Issue date 09/30/10, page 6), it was stated:

"Applications are sought in addressing gaps in our current understanding of ionizing radiation and neutron emission. In general, gamma-ray and neutron emissions from special nuclear materials have been well characterized across various parameters including magnitude, energy, and time. Gaps persist in the understanding of joint probability distributions, for example when considering correlated neutron emissions across energy and angle or correlations between prompt gamma-ray and neutron emissions.\} Other gaps in nuclear data are needed to fully exploit photo fission and nuclear resonance fluorescence signatures. Applications are sought that address these data gaps and clearly articulate their relevance to developing systems or methods for nonproliferation missions.” 
This section consists of three parts. First, we will examine the possible sensitivity of two neutron correlations in photofission to the presence of fissionable material. Second, we will discuss how such correlations can be studied with currently available broad spectrum pulsed bremsstrahlung beams. And lastly, we will address the large improvement that the availability of a combination of a CW beam and a photon tagger will have in performing these measurements. Such a study of the effect of two neutron correlations in photofission has not, to our knowledge, been performed to date.

\subsubsection{Two neutron correlations in photofission}

The technique of active interrogation, using both photons and neutrons, has shown considerable promise in the detection and quantification of special nuclear materials. One such approach involves inducing fission in a sample containing actinides, and measuring secondary emissions. Such a technique raises the signal orders of magnitude over passive techniques, decreasing detection limits and measurement time. For any target thicker than a few $\mathrm{mg} / \mathrm{cm}^{2}$, the fission fragments are not easily detectable due to their heavy ionization loss and resulting short range when traversing the target. Thus, the focus of such work has been on the detection of the highly penetrating photons and neutrons. The four broad classes of signatures under study include prompt gamma rays, delayed gamma rays, prompt neutrons, and delayed neutrons. Such studies seek to exploit the unique properties of the fission process, such as energy and time of the emissions, to distinguish fissionable nuclear materials from those which generate background emissions. In this example, we examine the possibility of kinematical correlations between neutrons emitted in a fission event, and explore the possibility that such effects can provide an unambiguous signature for fissionable material.

It has been shown experimentally that, to a very good approximation, recoiling fission fragments emit neutrons isotropically in their center of mass with a statistical energy distribution [BudtzJorgenson 1988]. A fully accelerated fission fragment travels with a speed of about $4 \%$ of the speed of light, $c$. In the center of mass of the fragment, a typical $1 \mathrm{MeV}$ neutron travels with a speed of about $4.6 \%$ of $c$. Thus, the neutron energy spectrum measured in the laboratory is a convolution of the "intrinsic" spectrum which the excited fission fragment emits, and the kinematical effects due to the fragment recoil. Qualitatively, one would expect that the higher end of the fission neutron energy spectrum would correspond to kinematics in which the neutron is emitted along the direction of recoil of the fission fragment. Since the two fission fragments are emitted approximately back to back, in the scenario where each fragment emits one neutron, one might expect a preponderance of back to back neutrons over parallel neutrons when both neutrons are from the higher energy range of the spectrum.

These effects were examined in a Monte Carlo simulation employing the following assumptions:

1. The fission fragment mass distribution was sampled uniformly between $85<A<105$ and 130 $<A<150$.

2. A fixed amount of total kinetic energy, $175 \mathrm{MeV}$, is given to the two fission fragments in a manner consistent with energy and momentum conservation. 
3. Neutrons are emitted isotropically in the center of mass of the fully accelerated fission fragments with an energy distribution given by:

$$
N(E)=E^{1 / 2} \exp (-E / 0.75)
$$

This reproduces the fission laboratory neutron energy distribution as measured with $(\mathrm{n}, \mathrm{f})$.

4. The fission fragment angular distribution is sampled in both polar angle $(\theta)$ and azimuthal angle $(\varphi)$ for either $K=0$ or $K=1$ as described in reference [Ratzek 1982], and the neutrons were given the appropriate kinematic boost.

Under the assumption that each fission fragment emits one neutron, the resulting asymmetry between parallel and anti-parallel neutrons is shown in Figure 5-10, where the $x$ axis is the energy cut placed on the energies of each of the two neutrons. As can be seen from the figure, the ratios of the anti-parallel versus parallel yields are quite large, particularly for higher neutron energies. It is worth emphasizing that this effect is a reflection of the fission process, and therefore holds promise of providing a unique signature for fissionable material. Furthermore as described below, the kinematics of such a measurement can be chosen so that any two neutron signal where both neutrons are of sufficient energy, can be unique to photofission.

\subsubsection{Measuring two neutron correlations with a pulsed beam}

Here, we examine the possibility of measuring two neutron correlations in the fission process using a pulsed electron beam. To make the discussion more concrete, we take the specific case of the High Repetition Rate Linac (HRRL) currently in operation at the Idaho Accelerator Center and a ${ }^{235} \mathrm{U}$ target. In the section following this, we investigate a possible experimental design using the CW accelerator described in Section 5.8 below.

A driving feature in the design of an experiment to measure the emission of two neutrons in the photofission process arises from the need to ensure that the two neutrons originate from the same fission. In particular, the ratio of trues/(accidentals + trues) is proportional to $1 / N_{f}$ where $N_{f}$ is the number of fissions in the target per pulse. This means that the experimental parameters must be such that one has approximately one fission per pulse. Typical pulse-widths for the HRRL are $20 \mathrm{~ns}$ and peak currents are $10 \mathrm{~mA}$. This gives $1.25 \times 10^{9}$ electrons/pulse. We are interested in the fission process. Given the photofission cross section (see Figure 5-11), the optimal beam energy is clearly above $6 \mathrm{MeV}$.

Since the process of interest involves photofission followed by the emission of two neutrons, the direct knockout of two neutrons without fission will be a background process which, if present, will dilute the signal. The cross section for the direct two neutron process is shown in Figure 5-12. As can be seen from the figure, keeping the photon beam energy below about 12 $\mathrm{MeV}$ will eliminate this background. 


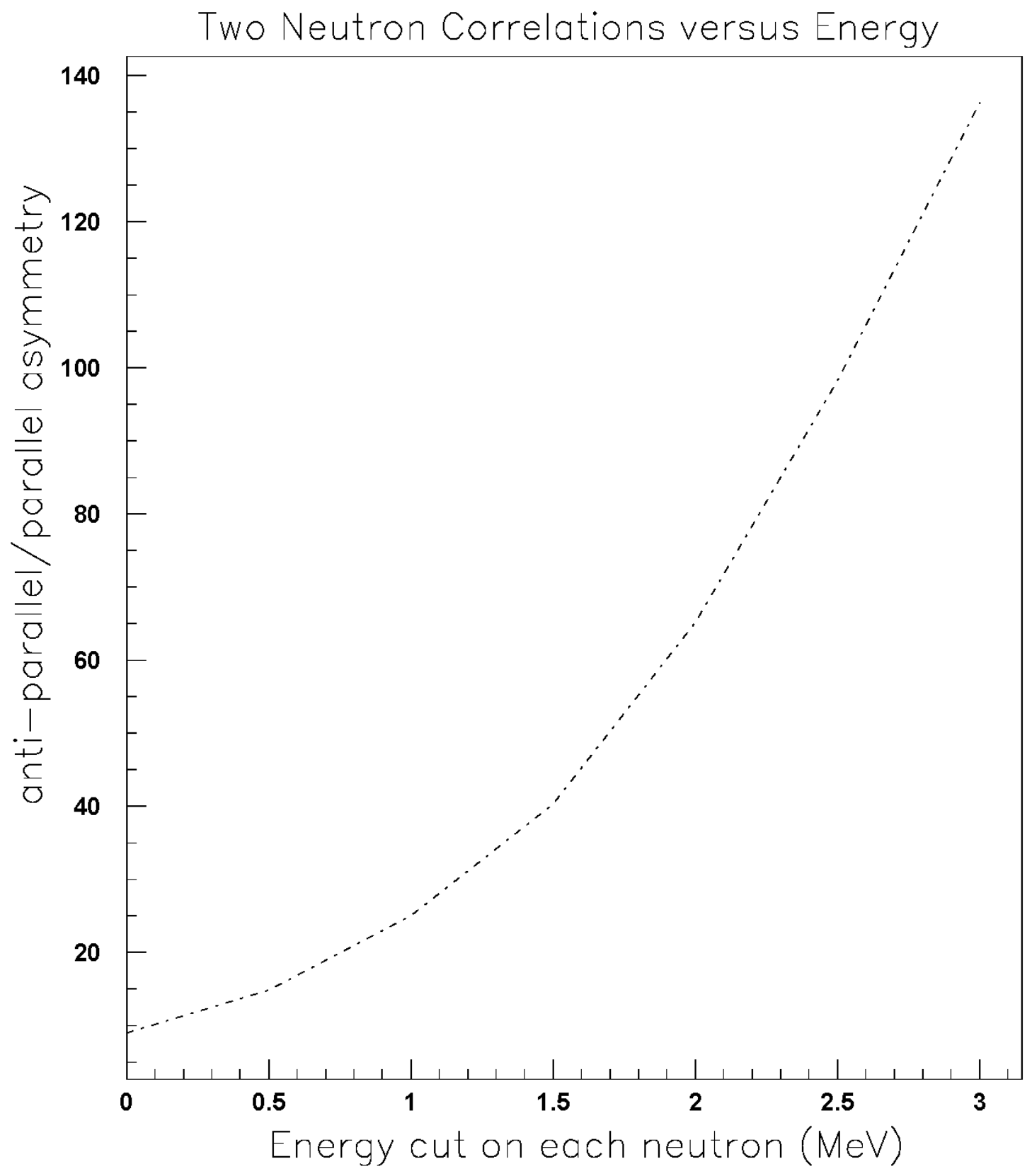

Figure 5-10. Ratio of neutrons emitted back-to-back versus parallel in the fission model described in the text. 


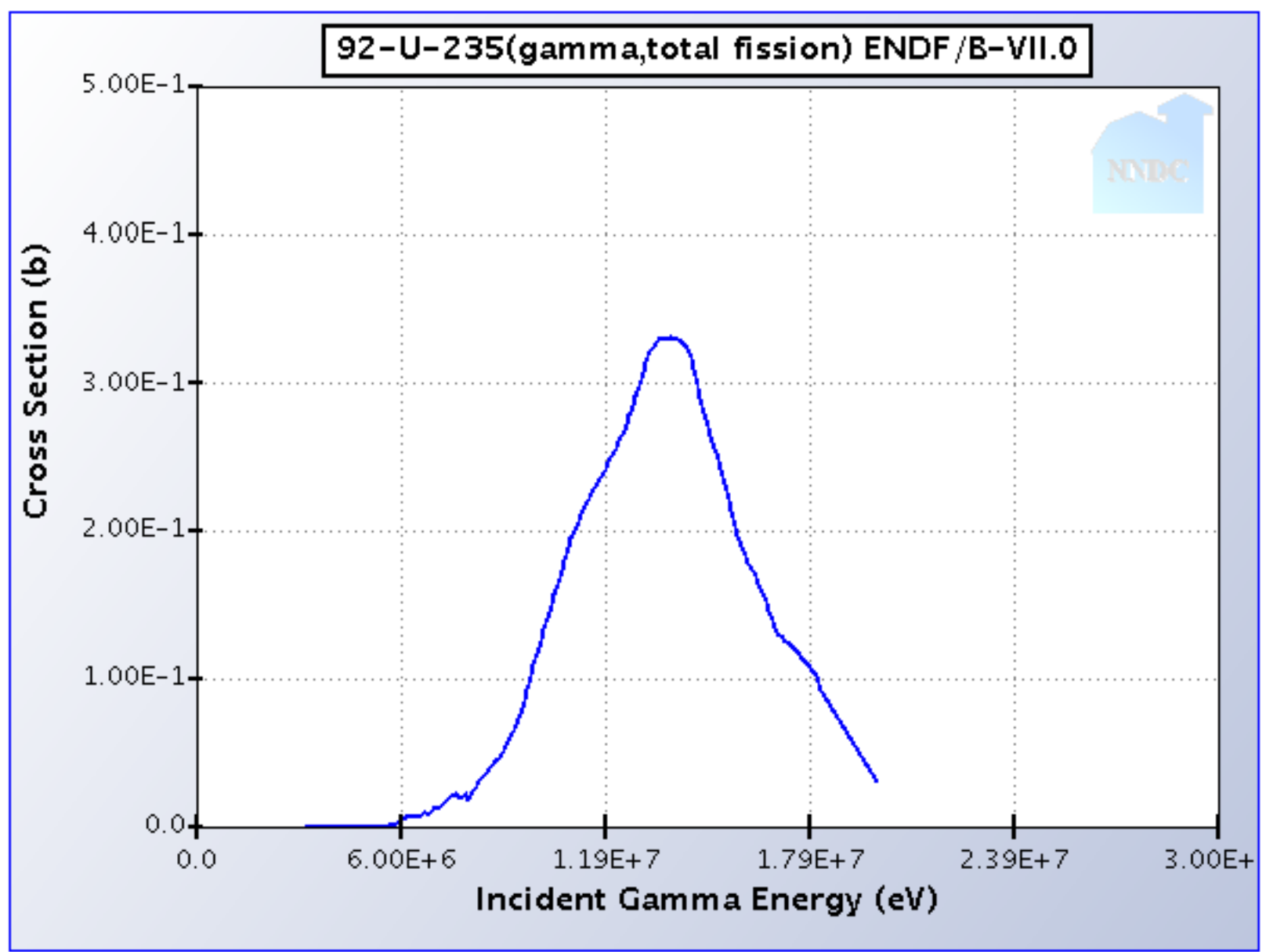

Figure 5-11. The photofission cross section for ${ }^{235} U$.

A 7-MeV bremsstrahlung endpoint energy meets the necessary criteria for the measurement: a low fission rate (i.e. 1 fission/pulse), and no direct two-neutron knockout. A low endpoint energy also has the advantage of generating fewer background neutrons from beamline elements -particularly higher energy neutrons.

Figure 5-13 shows the photon yield for a 7-MeV electron beam incident on an aluminum bremsstrahlung radiator, calculated using the formulism of [Matthews 1973]. In the 6-7 MeV photon energy range, the calculated yield is approximately 0.05 photons/electron/MeV/radiation length. For a $10^{-3}$ radiation length aluminum radiator (90 microns), one obtains $6.3 \times 10^{4}$ photons/pulse in the range of 6-7 MeV. With the fission cross section in the 6-7 MeV photon energy range taken to be $7 \times 10^{-27} \mathrm{~cm}^{2} /$ atom, the condition for 1 fission per pulse is as follows:

$$
1 \text { fission/pulse }=\left(\# \text { atoms } / \mathrm{cm}^{2}\right) \times\left(7 \times 10^{-27} \mathrm{~cm}^{2} / \text { atom }\right) \times\left(6.3 \times 10^{4} \text { photons/pulse }\right)
$$

This gives a required target thickness of not significantly more than $2.3 \times 10^{21}$ atoms $/ \mathrm{cm}^{2}$, or a $470 \mu \mathrm{m}$ target. While such a target is suitable for purposes of studying two neutron correlations 
in the laboratory, one is unlikely to encounter such a thin target when attempting to detect special nuclear materials in the field. In the next section, we will argue that this is not a problem in the context of a CW beam.

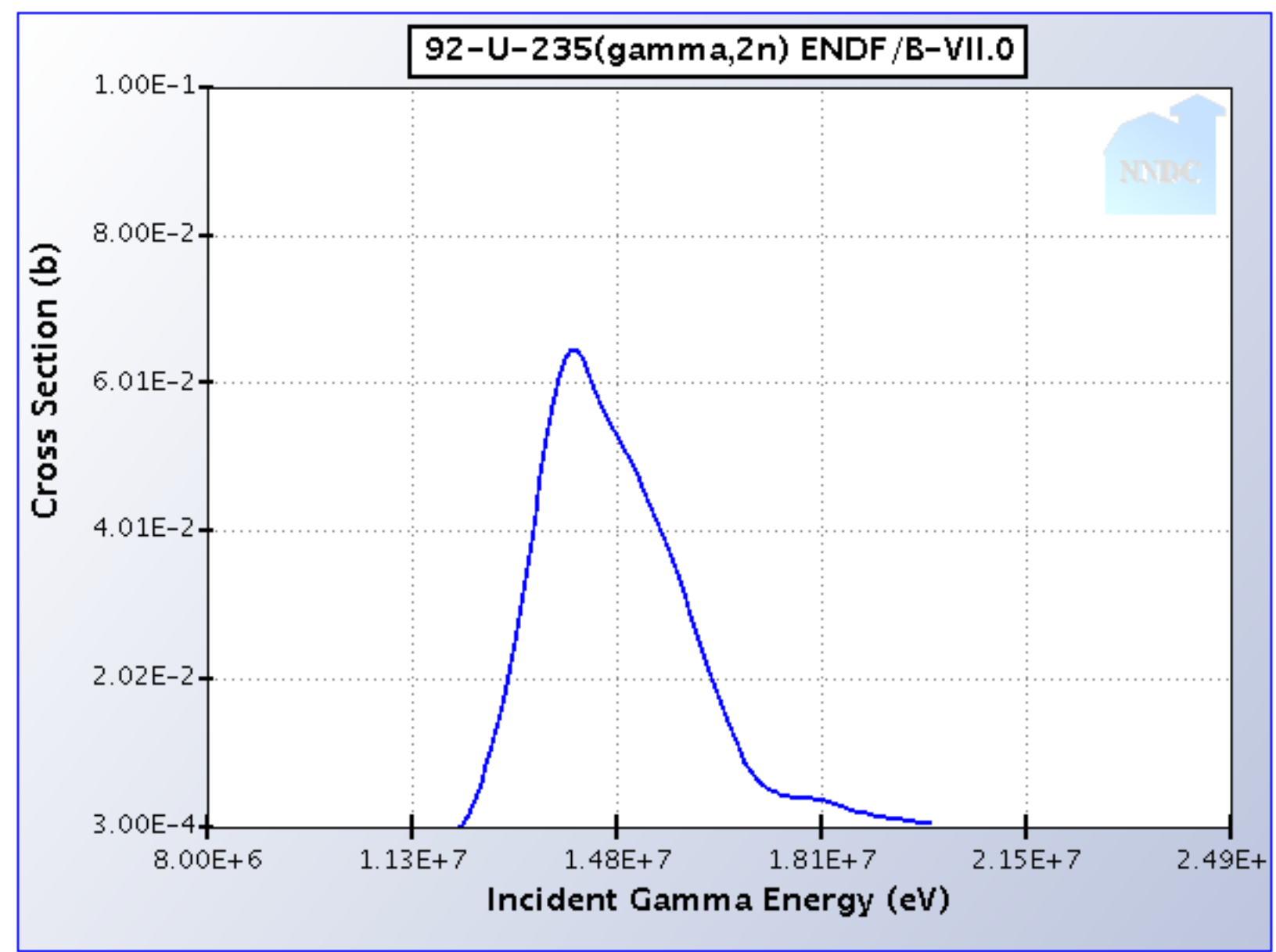

Figure 5-12. The direct two neutron emission cross section for ${ }^{235} U$. 


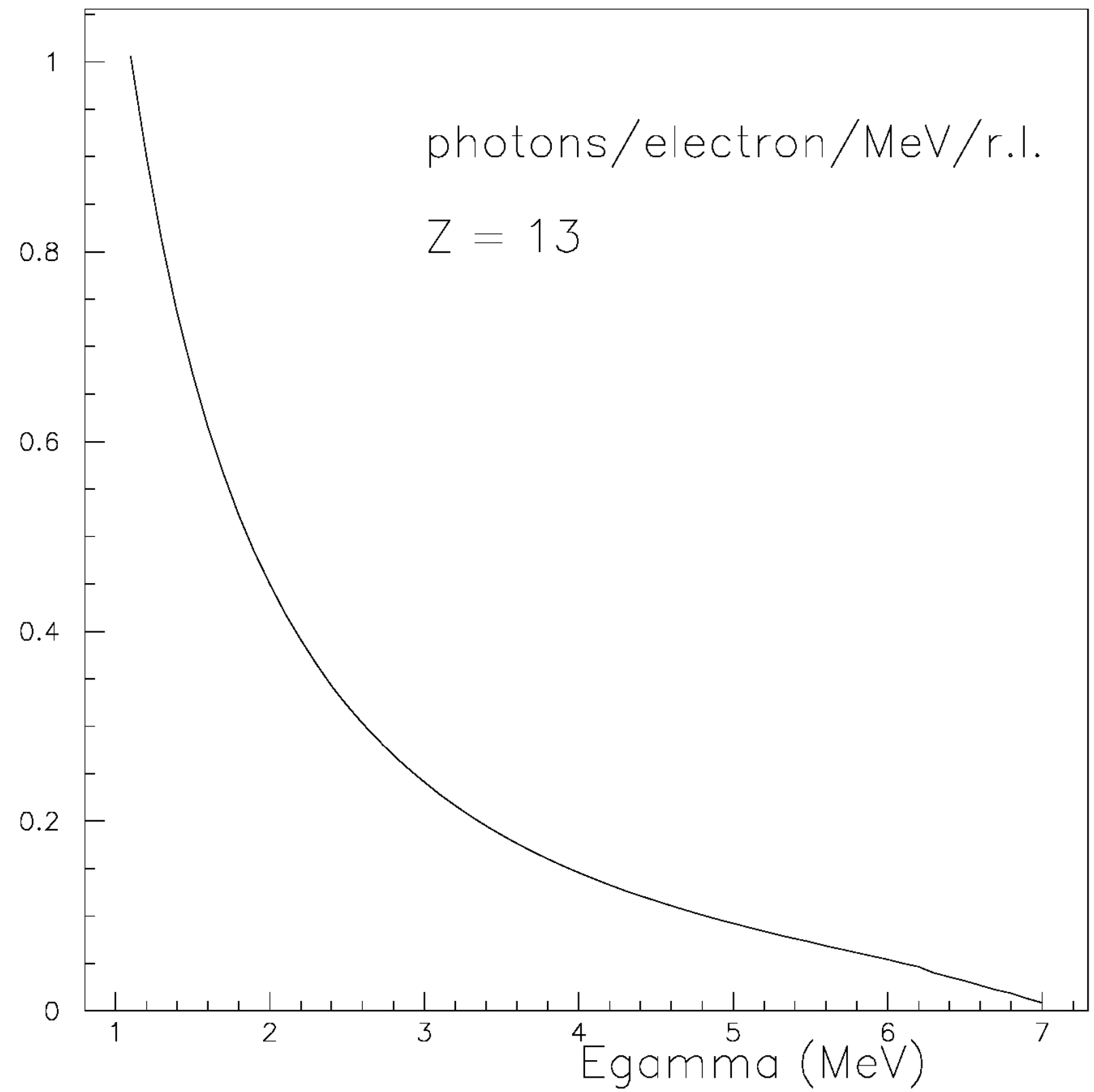

Figure 5-13. Calculated bremsstrahlung photon yield for a $7 \mathrm{MeV}$ electron beam.

With a pulsed beam, neutrons can be distinguished from background photons via the time of flight method. Figure 5-14 shows such a time of flight spectrum for the photodisintegration of the deuteron, taken from a previous experimental run on the High Repetition Rate Linac. The hatched histogram is for an $\mathrm{H}_{2} \mathrm{O}$ target and the solid line is for a $\mathrm{D}_{2} \mathrm{O}$ target. As can be seen from the spectrum, the neutrons are clearly distinguishable. 


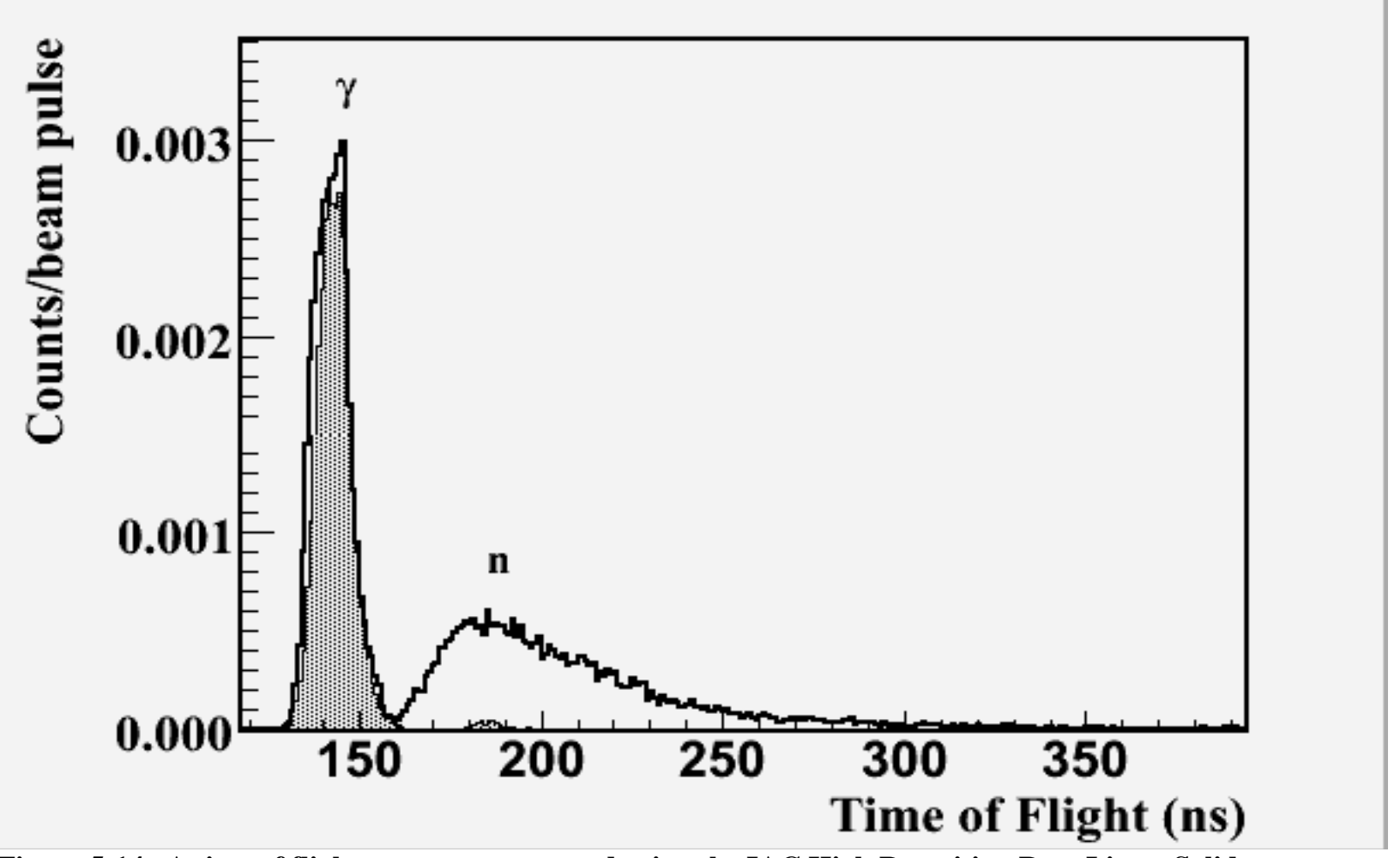

Figure 5-14. A time of flight spectrum measured using the IAC High Repetition Rate Linac. Solid line is for a $\mathrm{D}_{2} \mathrm{O}$ target and clearly shows the distribution of neutrons produced by photodisintegration of the deuteron. The hatched histogram is for $\mathrm{H}_{2} \mathrm{O}$ and shows a prominent gamma-ray "flash" distribution, but relatively little yield over larger flight times.

To estimate rates, we assume the following measurement parameters:

1. 16 plastic scintillator detectors $15 \times 88 \mathrm{~cm}^{2}$ in area with intrinsic efficiency of $25 \%$. These detectors can be instrumented with photomultiplier tubes at each end to give position information on the order of $\pm 5 \mathrm{~cm}$.

2. Detectors $2 \mathrm{~m}$ from target.

3. $1000 \mathrm{~Hz}$ repetition rate.

4. 2.2 neutrons/fission.

5. $1 \mathrm{MeV}$ neutron energy cut efficiency $=0.65$.

Under these conditions, one obtains 750 counts/day.

If the asymmetries are as large as the previously-presented model suggests, a pulsed beam will be more than adequate to establish the feasibility of using this effect in the detection of special nuclear materials. However, in the context of using this in a practical device in the field, the low duty factor of the beam presents significant challenges to its practicality. This situation changes with the availability of CW beam. 


\subsubsection{Measuring two neutron correlations with a CW beam}

Here, we consider the analogous experiment with a CW beam and a photon tagger instrumented with 60 tagging counters, each counting at $1 \mathrm{MHz}$. In this scenario, the photon flux is very similar to the case considered above for a pulsed beam. However, rather than being constrained to 1 fission per pulse (i.e. one fission per millisecond), the target can be thick enough such that one has 1 fission per microsecond. This fission rate enables one to relatively unambiguously identify the coincidence of a fission event with a given electron in a tagging counter. As a consequence, the target can be much thicker. An actinide target which is several centimeters thick, as one might encounter in the field, will not confound the measurement with accidentals due to the high fission rate, as was the case with a pulsed beam. Further, the measurement time for a given statistical precision is decreased by orders of magnitude. Thus the availability of a CW electron beam has the potential to enable the measurement of this effect on a time scale of a fraction of a minute. This huge advantage of a continuous wave electron beam capability is but one example of the improvements in experimental capabilities it would provide.

\subsection{The CW microtron design}

As mentioned previously, several techniques for the production of monochromatic gamma rays have been employed. Positron annihilation in flight is one such method which has been quite successful. In this technique, an electron linac is used to produce positrons which are then accelerated and impinge upon a thin annihilation target. The forward going annihilation photons are relatively monochromatic, but are superimposed upon a bremsstrahlung spectrum which must be subtracted off. The size of this instrumentation and the complexity of the measurements makes it an unlikely candidate for extensive use in special nuclear materials detection. Similarly, an electron storage ring fed by a linear accelerator can produce a CW beam, which can be extracted and used with a photon tagger, but again at the cost of a great deal of infrastructure. We argue that the microtron design, on the other hand, compares favorably with these technologies both in terms of cost and size.

Compact electron accelerators in the multiple $\mathrm{MeV}$ energy range using microwave driven linear multi-cavity structures are widely available. Figure 5-15 and Figure 5-16 show a commercial S band microwave linac structure. All but superconducting linacs run at low duty factors (the fraction of the time the RF power and beam are on), generally less than $1 \%$. This is because the high accelerating potentials along the structure require peak microwave powers on the order of several MW. Neglecting the small amount of energy carried out of the accelerator by the electron beam, this microwave energy is dissipated as heat in the structure. To make this method thermally practical, low duty factors are necessary as this allows high peak powers to be achieved using pulse techniques that require only modest average powers. Furthermore, high duty factor high power microwave sources at the several MW power level are not available and would need exceedingly large amounts of wall power if they were. These limitations mean that the standard accelerator produces beams that occur in pulses of order a few microseconds 
repeated at rates of several to several hundred $\mathrm{Hz}$, which precludes many important nuclear measurements such as those described here.

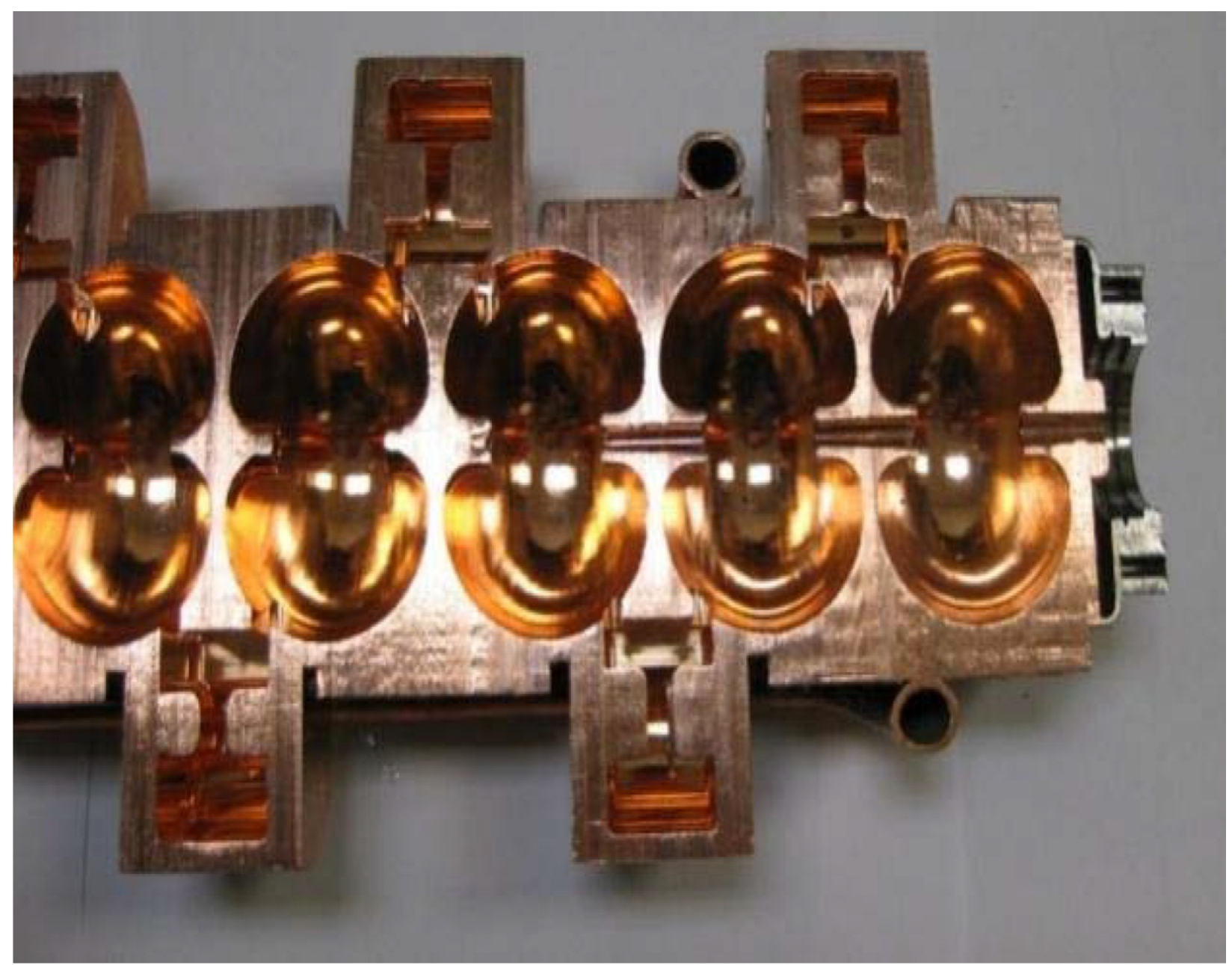

Figure 5-15. Cross section of a linac RF cavity with multiple cells. 


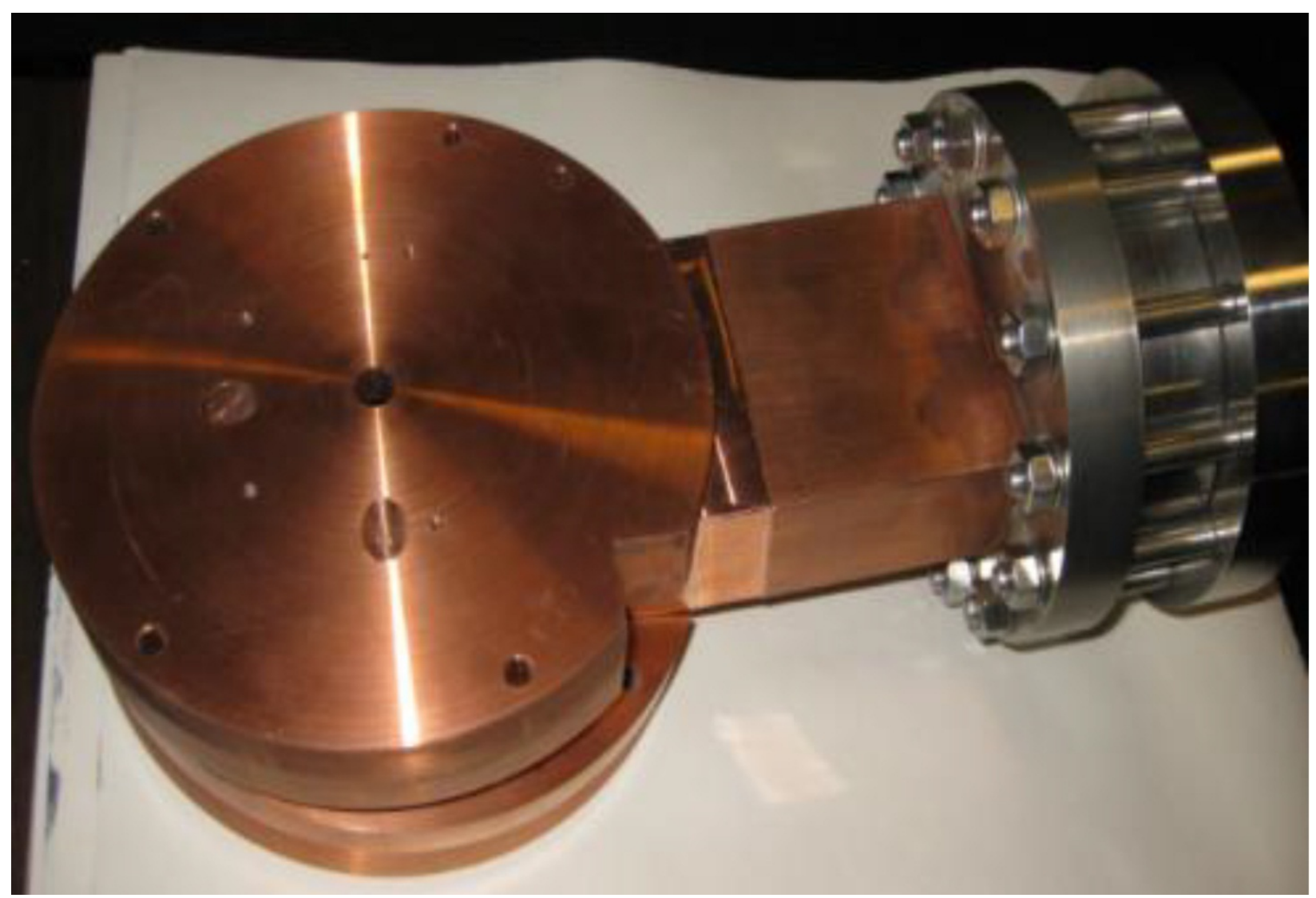

Figure 5-16. A single cell S-band microtron cavity.

In the microtron, magnets guide the beam in such a way as to return it to the microwave structure in a resonant series of $N$ orbits so that the same accelerating structure (and same microwave power) is used $N$ times, as indicated in Figure 5-17. For the same input power, the duty factor is increased $N$ times with the same final beam energy. Typically $N$ is $10-50$ and depends primarily on the size of the guide magnets. The principle limitation now becomes the size of the magnet system required for a given final energy, given the energy increment added on each orbit by the accelerator structure. In the energy range of interest here, the magnets required are relatively modest and if one or at most a few cavities are used in the accelerator structure, microwave power production and thermal management become practical for a 10 to $20 \%$ duty factor machine in the 5 - $20 \mathrm{MeV}$ range. 


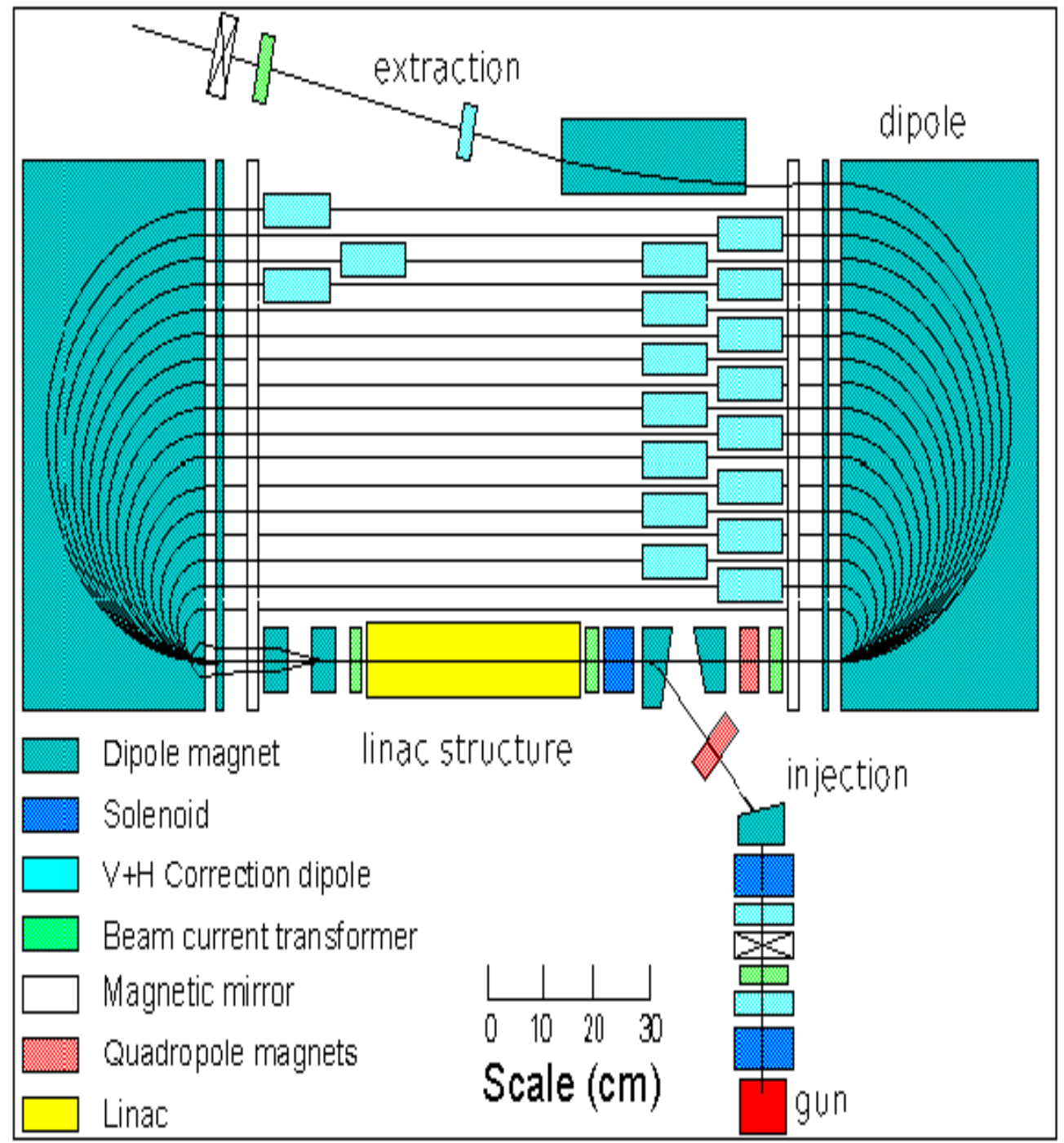

Figure 5-17. Schematic layout of a general racetrack microtron. Here the electron beam is initially injected from right to the left and then it passes through the linac structure. Because the beam at this point does not have enough momentum to make it past the linac structure due to its small bending radius, it is then bent 180 degrees at the left dipole and passes through the linac structure again. All subsequent orbits are then counterclockwise.

Development of this compact microtron could follow a phased approach, with the possibility of obtaining useful physics results at the end of the first phase. The first phase would comprise construction of a $7-\mathrm{MeV}$ microtron with a duty factor of about $10 \%$. To realize this duty factor, a single cell RF cavity similar to the ones shown in Figure 5-15 and Figure 5-16 would be used. By feeding an RF input power of about $10 \mathrm{~kW}$ average ( $100 \mathrm{~kW}$ peak) to the single cell RF cavity, the length of the RF and the beam pulse would be increased step by step until about $250 \mu$ s at a repetition rate of about $400 \mathrm{~Hz}$ is obtained, corresponding to a duty factor of $\sim 10 \%$. For the RF input power supplied, the gradient in the RF cavity will be 3 to $4 \mathrm{MV} / \mathrm{m}$ and the energy gain per turn will be 200 to $300 \mathrm{keV}$. After about 30 turns, the beam energy will be increased to 6 to $9 \mathrm{MeV}$. The significance of this energy range is that it includes the threshold region for photofission, so that measurements probing this response region could begin early in the design implementation. 
The second development phase would involve upgrading the RF cavity to construct a $20 \mathrm{MeV}$ microtron with a duty factor of $20 \%$. By using an RF cavity with multiple cells, one can reach a higher energy gain per turn of $800 \mathrm{keV}$ to supply $20 \mathrm{MeV}$ with 25 turns. The RF cavity would be modified to reduce the operating peak RF power down to $11.3 \mathrm{~kW}$. After improving the cooling channels of the RF cavity, the length of RF macropulse would be increased gradually until about $500 \mu$ s is obtained, which corresponds to a $20 \%$ duty factor. Attaining this duty factor at the IAC would require improvements to cooling channels for the electron gun and injection linac. Since the net peak RF input power is only $11 \mathrm{~kW}$ and the average beam current needed for the photon tagging program is only about $10 \mathrm{nA}$, we expect that beam quality dilution effects such as beam loading, wakefields, space charge effects, and synchrotron radiation would not be large. The 20 $\mathrm{MeV}$ energy at this stage would enable measurements of photonuclear cross sections in the region of the peak of the giant dipole resonance in heavy nuclei.

An example of commercially available end magnets for a microtron is shown in Figure 5-18. The length of the racetrack configuration is on the order of three meters. One technical challenge involves the fact that the first pass in the microtron has the tightest orbit, and could potentially not clear the accelerating cavity. This issue is driven by the desire to use a single accelerating cavity to keep the power dissipation low and the overall design of the microtron compact, as mentioned above. By installing bucking coils with an opposing magnetic field inside of the end magnets, the magnetic field in the region of the first orbit can be sufficiently modified so that it physically clears the accelerating cavity. The magnetic field of the bucking coils will be much lower than the nominal values $(0.100 \mathrm{~T}$ for $6 \mathrm{MeV}, 0.333 \mathrm{~T}$ for $20 \mathrm{MeV})$. Therefore, the electron beam can be circulated without colliding with the RF cavity. 


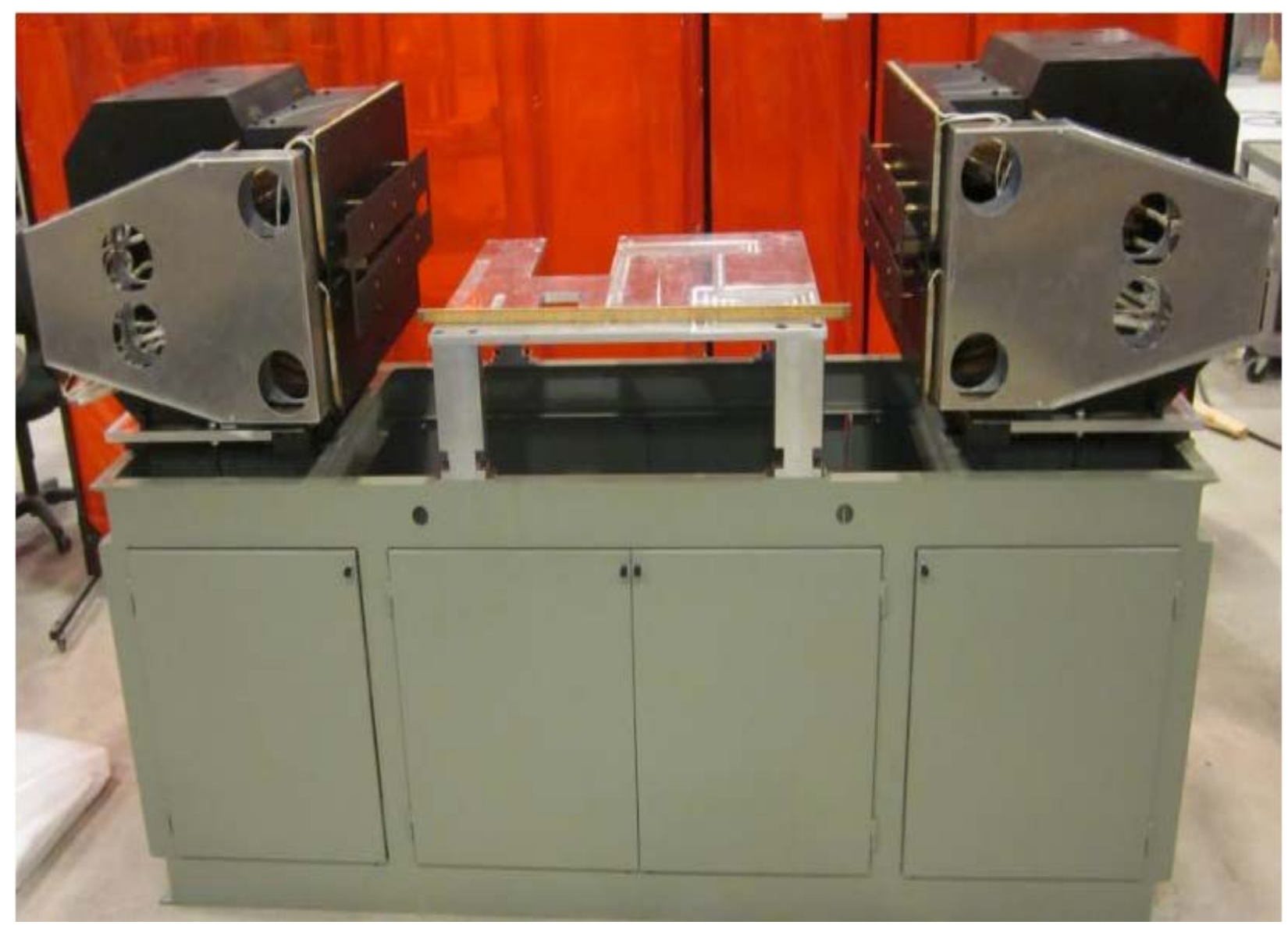

Figure 5-18. End magnets from a $50 \mathrm{MeV}$ microtron.

\subsection{Tagging Spectrometer: Ion-Optical Notional Design}

We have performed preliminary modeling of a notional photon-tagging spectrometer consisting of a dipole magnet and a set of focal plane detectors (e.g. simple scintillator paddles). The purpose of this modeling is not to delineate design features or performance of an optimized spectrometer, but rather to demonstrate that tagged-photon energy resolution consistent with the assumptions of the scenario analysis carried out in section 4 above are feasible in a reasonably simple spectrometer form-factor. The notional spectrometer design consists of the following elements:

- a bremsstrahlung radiator target;

- a dipole magnet, oriented with its bend plane either horizontal (i.e. parallel to the facility floor) or vertical; and

- a set of focal plane detectors.

The electron beam line, target radiator, dipole magnet, and focal plane detectors are all assumed to be vacuum coupled. Figure 5-19 illustrates an "overhead" view of the dipole magnet, an ensemble of electron trajectories, and the location of the focal "plane" (or focal surface) of the spectrometer. This surface is defined by the locus of points in space, $\mathrm{X}_{\mathrm{f}}(\mathrm{p})$, at which electrons emitted from the radiator target at constant momentum, $\mathrm{p}$, and varying angle of emission at the 
target, converge after traversing the magnetic field element. (In other words: To first order in the ion-optical transport properties of the system, a measurement of the position of the electron along the momentum-dispersion direction in this focal plane determines the magnitude of its momentum, regardless of the angle of emission at the target.) Table 5-1 summarizes properties of the spectrometer system.

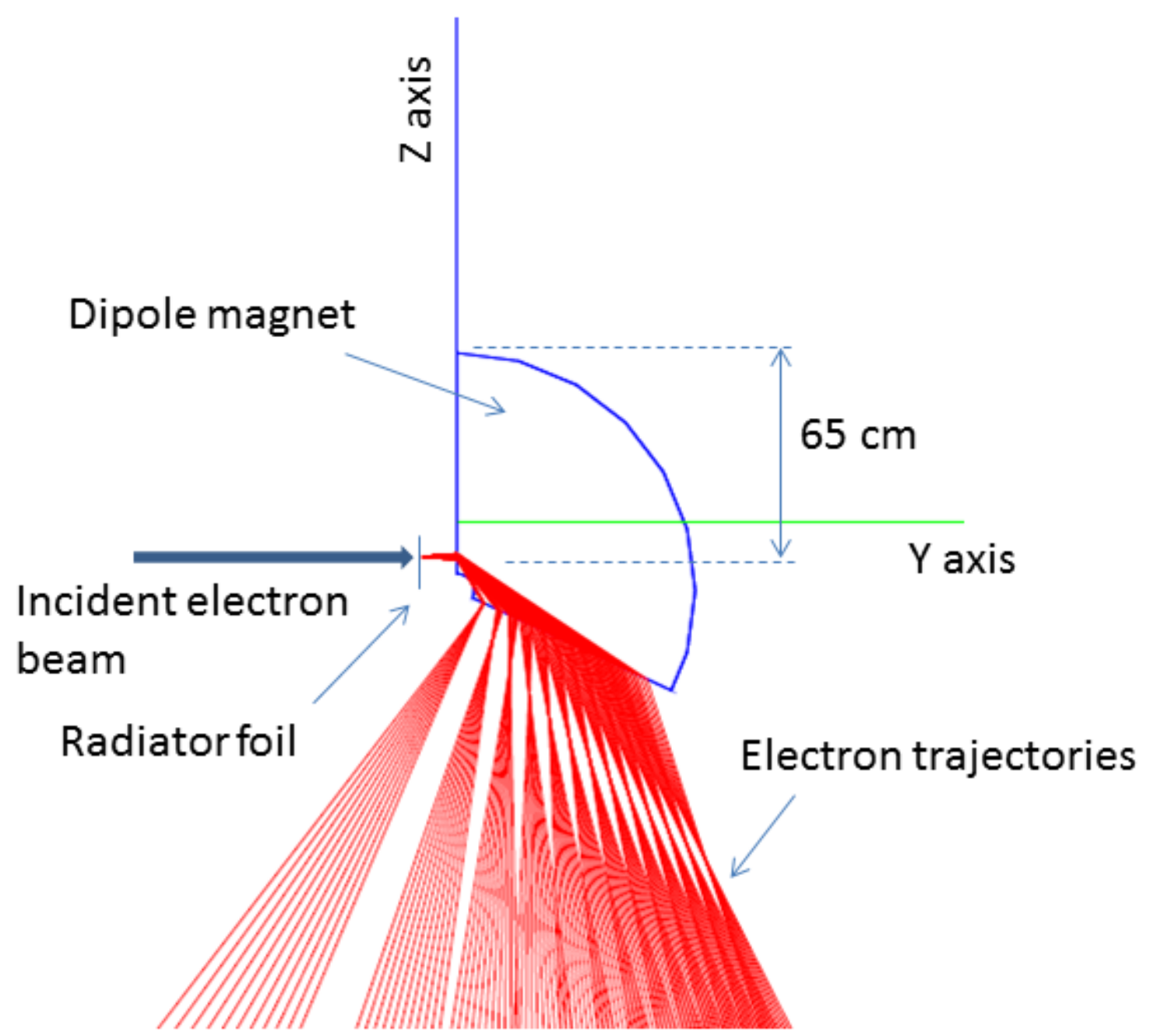

Figure 5-19. Overhead view of tagging spectrometer dipole, example electron trajectories, and focal plane position. Distinct bunches of electron trajectories correspond to different momenta. The locations of the narrow waists in the bunches following traversal of the dipole magnet define the focal "plane" (or focal surface). Note that the electron trajectories within the dipole are not plotted accurately in this figure; only the trajectories before and after the dipole correspond to the model of physical paths. 
Table 5-1. Properties of the notional tagging spectrometer.

\begin{tabular}{|l|l|}
\hline Property & Value \\
\hline Dipole inner radius & $5 \mathrm{~cm}$ \\
\hline Dipole outer radius & $70 \mathrm{~cm}$ \\
\hline Dipole bend angle & $115^{\circ}$ \\
\hline Dipole gap & $20 \mathrm{~cm}$ \\
\hline Dipole magnetic field & 0.09 Tesla (90 gauss) \\
\hline Electron kinetic energy bite & 2 to $14 \mathrm{MeV}$ \\
\hline Incident electron beam energy & $20 \mathrm{MeV}$ \\
\hline Tagged photon range & 6 to $18 \mathrm{MeV}$ \\
\hline Radiator material & Gold \\
\hline Radiator thickness & $10 \mu \mathrm{m}$ \\
\hline
\end{tabular}

Figure 5-20 displays the calculated energy resolution of the system as a function of electron kinetic energy. The calculation takes into account the distribution of post-bremsstrahlung electron angles, energy straggling in the radiator, and finite width of the focal plane detector array. The calculations do not take into account realistic dipole fringe fields. The radiator material and thickness were adopted in order to leverage previously-calculated electron emission distributions (energy and polar angle) documented in reference [Savran 2010]. Savran et al. consider a relatively thin radiator in order to minimize the occurrence of multiple electron scattering in the foil, an effect that degrades the tagged-photon energy resolution. Energy straggling in the 10- $\mu \mathrm{m}$ radiator introduces about $5 \mathrm{keV}$ (summed in quadrature) to the energy resolution, $\Delta \mathrm{E}$, plotted in Figure 5-20. The calculations of section 4 assume a $150-\mu \mathrm{m} \mathrm{Al}$ radiator. Assuming on the basis of comparisons in [Savran 2010] that the energy straggling scales roughly as the square root of the target thickness, and retaining the electron scattering properties in $\mathrm{Au}$ as a conservative estimate for an $\mathrm{Al}$ foil, the straggling contribution is on the order of $20 \mathrm{keV}$ for the scenario analyzed in section 4. This contribution, added in quadrature to the energy dispersion, will not significantly impact the main qualitative results of the calculation. The calculations indicate that the notional tagger design yields energy widths per channel of about $100 \mathrm{keV}$ or smaller over the tagged-photon energy range of 6 to $18 \mathrm{MeV}$, assuming a detector array consisting of relatively thin detectors (e.g. scintillating fibers) of width $2 \mathrm{~mm}$. At this focal plane detector width, approximately 350 detectors would be required to span the roughly 70-cm wide (in the y-direction of Figure 5-19) focal plane. Increasing the detector width to about $1 \mathrm{~cm}$ per detector (corresponding to narrow "finger" paddles of plastic scintillator) degrades the energy resolution to about $400 \mathrm{keV}$ per channel over most of the tagged energy range, and about $650 \mathrm{keV}$ at the low (electron) energy range of the focal plane. This range of resolutions is consistent with the assumptions of the photon-tagging active interrogation scenario analysis in section 4. 


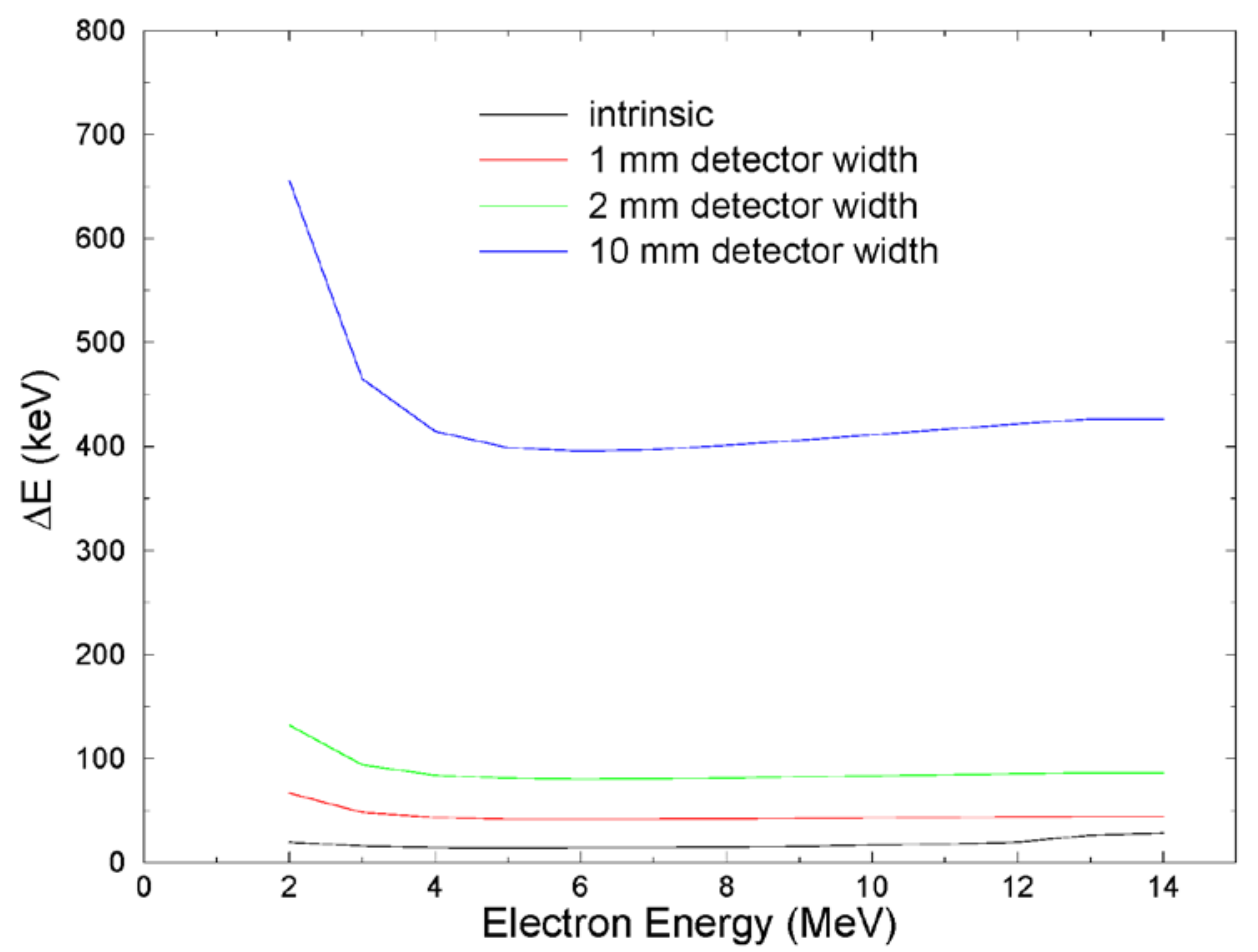

Figure 5-20. Calculated energy resolution of the notional photon tagging spectrometer as a function of postbremsstrahlung electron energy. The corresponding tagged-photon energy at 20-MeV endpoint energy runs from $6 \mathrm{MeV}$ (right side of plot, electron energy = 14 MeV) to $18 \mathrm{MeV}$ (left side of plot, electron energy = 2 $\mathrm{MeV}$ ). The curves correspond to different assumptions about the width of the focal plane detector. The "intrinsic" case represents the limit of the resolution for zero detector width. For $1 \mathrm{~mm}, 2 \mathrm{~mm}$, and $10 \mathrm{~mm}$ detector widths, the 71-cm focal plane extent in the $y$-axis direction (see axes in Figure 5-18) is spanned by 710,350 , and 71 detectors, respectively.

\subsection{Photon Tagger Design Considerations: Summary}

We have argued that a photon tagging system in the 10's of MeV range would provide an important facility for fundamental and applied physics measurements relating to the detection and identification of special nuclear materials. The nature of the photon tagging technique makes available photon beams which are precise in energy and in incident flux. Here, we have detailed newly developed experimental methods which have enabled absolute photon induced cross section measurements to be performed at the $1 \%$ level.

The coincidence techniques involved in photon tagging require the availability of CW (continuous wave) electron beams. While a number of options are available in this regard, we believe that the optimal accelerator design is that of the racetrack microtron. 


\section{Conclusions}

The Associated Particle Tagging (APT) project has explored the role of magnetic spectrometers as the enabling technology in next-generation sources for tagged-photon and tagged-neutron active interrogation (AI). We have examined two principle concepts in a computational and modeling-based feasibility study of ion-optical detection and spectrometry approaches for advanced tagged sources: (1) The application of magnetic spectrometry for detection and momentum-analysis of alpha particles in the D-T reaction offers substantial improvement in neutron tagging efficiency and equipment robustness for electronic neutron generators used for associated particle imaging (API). (2) Application of energy-tagged bremsstrahlung photons to AI offers a potentially powerful means of discriminating fissile from non-fissile materials by mapping the energy dependence of the photoneutron yield over the energy range 6 to $20 \mathrm{MeV}$, separating the relative contributions of the photofission and photoneutron (giant dipole) processes.

For the neutron API concept, PNNL evaluated detection and ion-optical spectroscopy of alpha particles emitted in the D-T or D-D reactions (with alpha-particle detection serving to "tag" the corresponding outgoing neutron in angle and energy) by constructing a Geant4 radiationtransport model of a notional neutron generator. This model provided a computational basis for bounding important properties of the tagged-neutron beam, including resolution in timing, angular direction, and energy. The solenoidal magnetic spectrometer design evaluated in this feasibility study shows a potential for 4.5 steradian tagged neutron solid angle, and further increases may be possible with optimization of the focal plane detector array design. Reconstruction of the neutron polar angle to within $0.4^{\circ}$ to $2^{\circ}$ (variable with angle) should be possible according to the simulated spectrometer. PNNL partnered with Dr. David Chichester of the Idaho National Laboratory (INL) to obtain technical guidance and expertise on the conceptual design of a realistic neutron generator incorporating the alpha-spectrometer concept.

The photon-tagging feasibility study included a thorough information-content analysis of the value-added for SNM detection of tagged energy resolutions two to three orders of magnitude finer than possible with broad-spectrum (i.e., untagged) bremsstrahlung photon beams. PNNL's computational study emphasized analytical and Monte Carlo modeling of detection of photoejected neutrons and photofission neutrons. We focused on quantifying the potential capability to discriminate the measured spectra of fast photoneutrons in the $\left(\mathrm{E}_{\gamma}, \mathrm{E}_{\mathrm{n}}\right)$ plane registered from fissile and benign target materials. PNNL collaborated with Dr. Daniel Dale of Idaho State University (ISU)/Idaho Accelerator Center (IAC) in the photon-tagging feasibility study, with the ISU/IAC providing accelerator physics assessments of continuous-wave (CW) infrastructure required to implement the tagging concept for a dedicated, fixed-installation active interrogation facility. In support of the infrastructure studies, PNNL carried out a preliminary study of a notional dipole-magnetic based approach to the ion optics required for a tagging spectrometer. This study demonstrated that tagged photon energy resolutions comparable to the range assumed for the photoneutron modeling studies should be feasible with a vacuum-coupled system consisting of beamline, bremsstrahlung foil target, dipole magnet, and focal-plane detector array. 
These initial scoping studies have yielded sufficient understanding of the potential performance of magnetic spectrometer-based tagged sources for AI applications to suggest that measurementbased validation studies of the computational assessments may be beneficial. A useful avenue for follow-on research of the next-generation tagged-neutron generator concept would involve experimental validation of the main conclusions of the neutron generator modeling. A validation campaign should begin with the fabrication of a demonstration focal plane alpha-particle detector array. This effort would comprise engineering design, fabrication, and testing of a prototype alpha-spectrometer system (including focal plane array and magnetic field elements) appropriate for incorporating in a D-T electronic neutron generator for API. The primary demonstration design objective would be to furnish a useful test-bed of the alpha-spectrometry concept in a reasonably compact form-factor. A prototype alpha-particle spectrometer would facilitate testing of the operational and robustness properties of a Si detector array in a form factor, and magnetic field environment, relevant to the proposed neutron generator design.

A possible notional design for the demonstration Si focal plane array is illustrated in Figure 6-1 below. The design follows [Wuosmaa 2007] and consists of a set of Si position-sensitive detector (PSD) strips, arranged in panels of approximately $2 \mathrm{~cm}$ width by $5 \mathrm{~cm}$ length, surrounding a square-cross section support tube. Position sensitivity in both the longitudinal (i.e. beam-axis) position and the azimuthal direction are required for the final neutron generator concept. For purposes of an initial performance validation, however, demonstration of the technique's polar angle reconstruction capability (an angle which maps directly to tagged neutron polar angle) could be achieved with a detector array yielding only crude position resolution in the longitudinal and azimuthal directions. The notional demonstration design illustrated in the figure features a 24-panel detector set providing 4-sided azimuthal coverage over a 30 -cm longitudinal span.

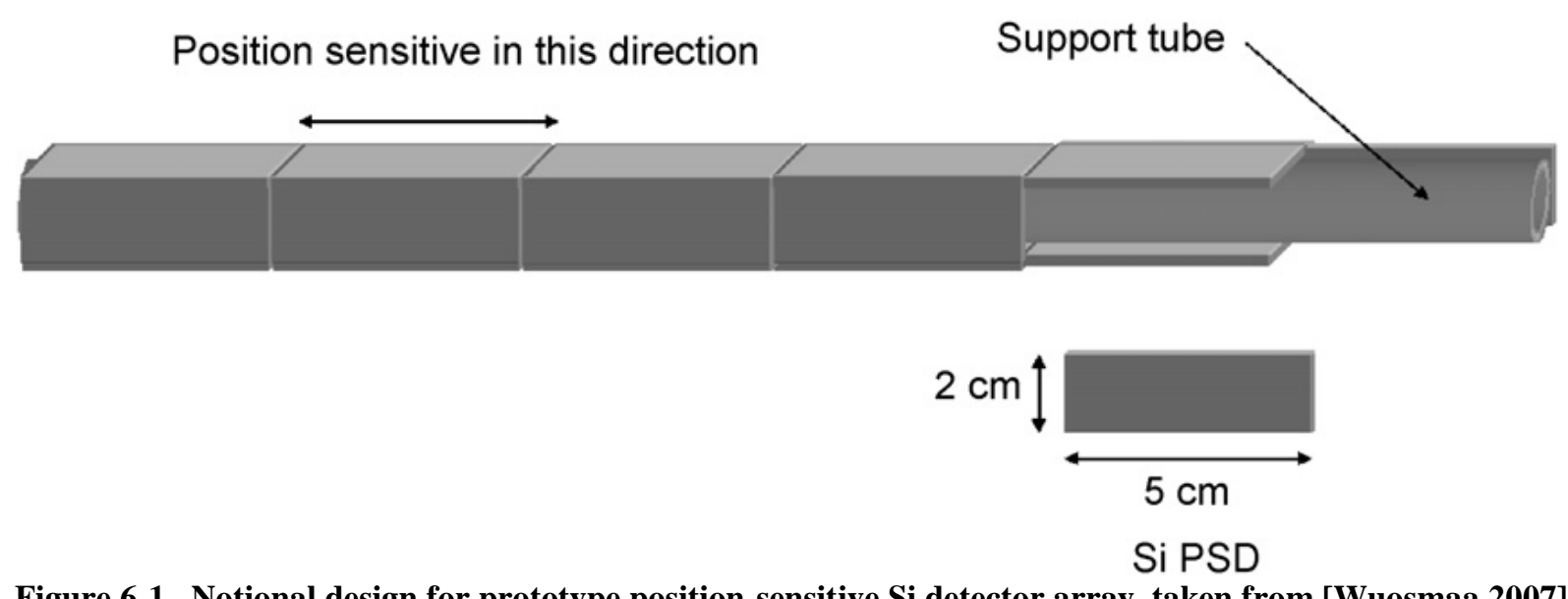

Figure 6-1. Notional design for prototype position-sensitive Si detector array, taken from [Wuosmaa 2007].

Possible follow-on research scope for the photon-tagging "information content" assessment should emphasize a set of measurement campaigns conducted at one or more beamline facilities. Two types of possible measurement campaigns would enable a meaningful test of the tagged photoneutron spectroscopy method for discriminating fissile from non-fissile materials: 
- Measurements at an existing tagged-photon facility offering tagged-photon coverage over the 6-20 MeV energy range of interest.

- Measurements at the HIGS facility at TUNL. This option provides a set of quasimonoenergetic beams (in pulsed operation) that would provide experimental "simulation" of the tagged-photon principle of operation.

In either case, a measurement campaign should consist of bombarding targets configured in simple, slab-target geometry with the photon beam, and registering coincident detection of outgoing fast neutrons. The primary measurement objective would be validation of the computational conclusions of the feasibility study, indicating that discrimination of fissile from non-fissile materials can be achieved by mapping the photoneutron emission in the $\left(E_{\gamma}, E_{n}\right)$ response plane. A possible tagging-facility candidate for the measurements would be the highresolution NEPTUN tagger at the Darmstadt facility in Germany. This facility provides tagged photon energies spanning the giant dipole resonance for neutron photoemission, e.g. about 10 $\mathrm{MeV}$ to $20 \mathrm{MeV}$. A reasonably simple neutron spectrometer (e.g. liquid scintillator with pulse shape discrimination for gamma-ray rejection, or a multi-slab PVT plastic scintillator time-offlight detector) could be deployed in the measurement campaign. A reasonable target set should include, at a minimum, depleted uranium, lead, iron, and aluminum targets, arranged in various "sandwich" configurations. Experiments at HIGS represent another option for mapping the neutron responses and physically simulating (via sequential "mono-energetic" photon beam measurements) the energy specificity of the tagged-photon method. The HIGS facility uses the Compton backscattering process inside a free electron laser cavity to generate intense, nearly mono-energetic, polarized, pulsed beams of gamma rays with tunable energies between 2 and 90 $\mathrm{MeV}$. In the 6 to $20 \mathrm{MeV}$ energy range relevant to this research, the collimated intensity on target is typically $10^{7}$ to $10^{8}$ photons per second for circular polarization with an energy spread FWHM of 5\%. The pulsed nature of the beam allows neutron time of flight measurements and aids in the discrimination between gamma rays and neutrons.

In summary, possible research paths following on from this feasibility study into ion-optical spectrometry concepts should address the following:

Neutron API: Design and fabricate a demonstration magnetic spectrometer for alpha-particle detection in a D-T neutron generator. Following fabrication and bench-testing of a (possibly limited-resolution) alpha-particle detector array, alpha-source and beamline-based demonstrations of the spectrometer concept can proceed. Beamline tests may use either an alpha-particle beam (subjected to elastic scattering from an appropriate target) or a deuterium beam (impinging on a deuterium or tritium target). Advanced stages of the technology development would include engineering design and fabrication of a full-up, next-generation API neutron generator incorporating a high-resolution alpha spectrometer. Higher-fidelity design modeling would implement a better model of a suitable solenoid, preferably based upon an existing solenoid of suitable performance. Key issues in the magnet design include specification of the required field uniformity over the axial and radial dimensions of the spectrometer and determination of the minimum solenoid length needed so that the fringing fields at the ends of the magnet do not significantly degrade the needed field uniformity. Another key effect is calculating the reduction of useable solid angle due to the coils and structure of the solenoid. 
Photon tagging: Plan and execute measurements in simple "slab-target" geometry to validate this feasibility study's computational evaluations of the potential for fissile/non-fissile discrimination via fast photoneutron emission, mapped over the ( $\left.E_{\text {gamma }}, E_{\text {neutron }}\right)$ plane. The establishment of a low-energy (6 to $20 \mathrm{MeV}$ ) tagging facility in the United States, dedicated to research in active interrogation applications of the tagged-photon approach, would provide a useful venue for pursuing these studies and for measuring nuclear cross section data required for high-fidelity radiation transport modeling in a variety of threat reduction applications. 


\section{Acknowledgements}

The project team gratefully acknowledges Dr. Bret Cannon for his thorough peer review of the report manuscript and many helpful suggestions. This research was sponsored by the Department of Energy Office of Nonproliferation Research and Development (NA-22). Pacific Northwest National Laboratory is operated for the U.S. Department of Energy by Battelle under contract DE-AC05-76RL01830. 


\section{References}

[Agostinelli 2003] S. Agostinelli et al, Nucl. Instr. and Meth. A 506 (2003) 250.

[API 1998] "Associated Particle Imaging (API)," Report DOE/NV/11718-223, Special Technologies Laboratory, Santa Barbara, Calif. (1998).

[Anthony 1991] I. Anthony et al., Nucl. Instr. and Meth. A301 (1991) 230.

[Argan 1984] P. Argan et al., Nucl. Instr. and Meth. 228 (1984) 20.

[Bethe 1934] H. Bethe and W. Heitler, Proc. Roy. Soc. Lond., A146 (1934) 83.

[Bianchi 1992] N. Bianchi et al., Nucl. Instr. and Meth. A317 (1992) 434.

[Blagus 2004] Blagus, S., Sudac, D., and Valković, "Hidden Substances Identification by Detection of Fast Neutron Induced $\gamma$ Rays Using Associated $\alpha$ Particle Detection," Nucl. Inst. Meth. Phys. Res. B 213 (2004) 434-438.

[Budtz-Jorgensen 1988] C. Budtz-Jorgensen and H.H. Knitter, Nucl. Phys. A490 (1988) 307.

[Chadwick 2006] M.B. Chadwick, P.O., M. Herman et al., ENDF/B-VII.0: Next Generation Evaluated Nuclear Data Library for Nuclear Science and Technology. Nuclear Data Sheets, 2006. 107: p. 29313060

[Chichester 2005] Chichester DL, M Lemchak, JD Simpson, “The API 120: A portable neutron generator for the associated particle technique,” Nucl. Instr. and Meth. B241 (2005), 753-758.

[Detemple 1992] P. Detemple et al., Nucl. Instr. and Meth. A321 (1992) 479.

[Dietrich 1988] Dietrich, S.S., Berman, B.L., Atlas of photoneutron cross sections obtained with monoenergetic photons. Atomic Data and Nuclear Data Tables, 1988. 38: p. 199-338.

[Ellis 2008] Ellis, K. J. and Shypailo, R. J., "COHN Analysis: Body Composition Measurements Based on the Associated Particle Imaging and Prompt-Gamma Neutron Activation Analysis Techniques," J. Radioanal. Nucl. Chem. 276 (2008) 79-83.

[Evans 2002] Evans, C. J. and Mutamba, Q. B., "The Use of Associated Particle Timing Based on the D + D Reaction for Imaging a Solid Object," App. Rad. Iso. 56 (2002) 711-716.

[Gordon 1990] Gordon, C. M. and Peters, C. W., "A Fast-Neutron Probe for Tomography and Bulk Analysis," App. Rad. Iso. 41 (1990) 1111-1116.

[Hollas 1990] Hollas, C. L. and Ussery, L. E., "An Application of the Associated-Particle Technique to Treaty Verification," Report LA-11876-MS, Los Alamos National Laboratory, Los Alamos, N.M. (1990).

[Kuznetsov 2004] Kuznetsov, A. V., et al., "Detection of Buried Explosives Using Portable Neutron Sources with Nanosecond Timing," App. Rad. Iso. 61 (2004) 51-57. 
[Lunardon 2004] Lunardon, M., et al., "Detection of Landmines by Using $14 \mathrm{MeV}$ Neutron Tagged Beams," App. Rad. Iso. 61 (2004) 43-49.

[Matthews 1973] J.L. Matthews and R.O. Owens, Nucl. Instr. and Meth. 111 (1973) 157.

[Mitra 1995] Mitra, S., et al., "Application of the Associated Particle Technique for the Whole-Body Measurement of Protein, Fat and Water by $14 \mathrm{MeV}$ Neutron Activation Analysis - A Feasibility Study," Phys. Med. Biol. 40 (1995) 1045-1055.

[Morris 1990] Morris, R. A., et al., "Treaty Verification Inspection Using Low-Technology Systems," Report LA-11811-MS, Los Alamos National Laboratory, Los Alamos, N.M. (1990).

[Nebbia 2004] Nebbia, G., et al., "Performance of a Tagged Neutron Inspection System (TNIS) Based on Portable Sealed Generators," Nucl. Inst. Meth. Phys. Res. A 533 (2004) 475-480.

[Oblozinsky 2000] Oblozinsky, P., Handbook on Photonuclear Data for Applications: Cross-seconds and spectra. 2000, IAEA: Vienna.

[Oblozinsky 2009] Oblozinsky, P., ENDF-6 Formats Manual. 2009, Brookhaven National Lab: Upton, NY.

[Owens 1990] R.O. Owens, Nucl. Instr. and Meth. A288 (1990) 574.

[Pascale 1985] M.P. de Pascale et al., Phys. Rev. C32 (1985) 1830.

[Ratzek 1982] R. Ratzek et al., Z. Phys. A, Atoms and Nuclei 308 (1982) 63.

[Rhodes 1992] Rhodes, E., et al., "APSTNG: Radiation Interrogation for Verification of Chemical and Nuclear Weapons," IEEE Trans. Nucl. Sci. 39 (1992) 1041-1045.

[Rhodes 1995] Rhodes, E., et al., "APSTNG: Associated Particle Sealed-Tube Neutron Generator Studies for Arms Control," Report ANL/ACTV-95/1, Argonne National Laboratory, Argonne, Ill. (1995).

[Savran 2010] D. Savran, K. Lindenberg, J. Glorius, B. Löher, S. Müller, N. Pietralla, L. Schnorrenberger, V. Simon, K.Sonnabend, C. Wälzlein, M. Elvers, J. Endres, J. Hasper, A. Zilges, “The low-energy photon tagger NEPTUN,” Nucl. Instr. and Meth. A613 (2010) 232.

[Sober 2000] D.I. Sober et al., Nucl. Instr. and Meth. A440 (2000) 263.

[Terasawa 1986] T. Terasawa et al., Nucl. Instr. and Meth. A248 (1986) 429.

[Teymurazyan 2006] A. Teymurazyan, Ph.D. thesis, University of Kentucky, 2006.

[Vogt 1993] Vogt JM, RE Pywell, DM Skopik, EL Hallin, JC Bergstrom, HS Caplan, KI Blomqvist, W Del Bianco, JW Jury, “The photon tagging facility at the Saskatchewan Accelerator Laboratory,” Nucl. Instr. and Meth. A324 (1993), 198.

[Wielopolski 2008] Wielopolski, L., et al., "Nondestructive System for Analyzing Carbon in Soil," Soil Sci. Soc. Am. J. 72 (2008) 1269-1277. 
[Wuosmaa 2007] Wuosmaa AH, JP Schiffer, BB Back, CJ Lister, KE Rehm, “A solenoidal spectrometer for reactions in inverse kinematics,” Nucl. Instr. and Meth. A580 (2007), 1290-1300. 